University of Wisconsin Milwaukee

UWM Digital Commons

Theses and Dissertations

$12-1-2016$

\title{
The Association of Nurses' Assessment and Certainty to Pain Management and Outcomes for Nursing Home Residents in Jordan
}

Mohammad Jamil Rababa

University of Wisconsin-Milwaukee

Follow this and additional works at: https://dc.uwm.edu/etd

Part of the Nursing Commons, and the Other Medical Specialties Commons

\section{Recommended Citation}

Rababa, Mohammad Jamil, "The Association of Nurses' Assessment and Certainty to Pain Management and Outcomes for Nursing Home Residents in Jordan" (2016). Theses and Dissertations. 1406.

https://dc.uwm.edu/etd/1406

This Dissertation is brought to you for free and open access by UWM Digital Commons. It has been accepted for inclusion in Theses and Dissertations by an authorized administrator of UWM Digital Commons. For more information, please contact open-access@uwm.edu. 


\title{
THE ASSOCIATION OF NURSES' ASSESSMENT AND CERTAINTY TO PAIN
}

MANAGEMENT AND OUTCOMES FOR NURSING HOME RESIDENTS IN JORDAN

by

\author{
Mohammad J. Rababa
}

\begin{abstract}
A Dissertation Submitted in
Partial Fulfillment of the

Requirements for the Degree
\end{abstract}

\author{
Doctor of Philosophy \\ in Nursing
}

at

The University of Wisconsin-Milwaukee

December 2016 


\begin{abstract}
THE ASSOCIATION OF NURSES' ASSESSMENT AND CERTAINTY TO PAIN MANAGEMENT AND OUTCOMES FOR NURSING HOME RESIDENTS IN JORDAN
\end{abstract}

by

\author{
Mohammad Rababa
}

The University of Wisconsin-Milwaukee, 2016

Under the Supervision of Dr. Christine Kovach

Despite advances in dementia care Pain in people with dementia is still under recognized, underestimated, and undertreated because of poor assessment, poor treatment, and factors relating to nurses' critical thinking and decision-making skills. Unrelieved pain leads to many serious negative health outcomes in people with dementia. The purpose of the study was to examine temporally based relationships between change in behavior, the nurses' level of certainty regarding pain, assessment scope and outcomes of pain and agitation. Relationship between severity of dementia, comorbid burden, ability to verbally self-report symptoms and patient outcomes of pain and agitation was also investigated.

A Convenient sample of 78 nursing home residents with dementia was selected from 5 nursing homes in Amman, Jordan. The average age of the participants was 72.52 years and $56.0 \%$ of them were men. Nurses' level of certainty regarding suspected pain was examined with one item Likert Scale. Scope of pain assessment was coded and collected directly from change tracking sheets. Pain was measured by Discomfort Scale for Dementia of Alzheimer's Type. Agitation was measured by Wisconsin Agitation Inventory tool. Severity of dementia was measured by the Mini Mental State Examination. Comorbid burden was measured by Cumulative Illness Rating Scale for Geriatrics and obtained from medical records. Ability to 
verbally self-report symptoms was measured by Communication of Need - Dementia Alzheimer's Type tool.

The findings of the original study are consistent with the RCP model, but inconsistent with literature that shows that agitation is caused by multiple factors.

This study found an excessively high percentage of variances accounted for by nurses' level of certainty due to potential measurement errors, the preliminary nature of this study, and potential unexpected uncontrolled confounding variables. The findings that comorbid burden and ability to verbally self-report symptoms are significant predictors of patient outcomes are consistent with previous studies. The finding that the relationship between comorbid burden and patient outcomes could not be explained by ability to verbally self-report symptoms is consistent with previous studies. The discussion of results and the interpretation of findings should be presented with caution and replicated before being applied to practice or theory derivation. While findings must be replicated with other samples, this study may provide a new understanding of the relationship between nurses' certainty, assessment scope, and patient outcomes for PWD. Improved understanding of comorbid burden and the ability of PWD to verbally self-report symptoms and how they relate to the problem of unrelieved pain in PWD is crucial. 
CCopyright by Mohammad Rababa, 2016 All Rights Reserved 


\section{DEDICATION}

All praises and gratitude be to Allah (God), whom out of his bounty this work was completed, and may the peace and blessings of Allah be upon his last messenger

Muhammad. This dissertation is dedicated to my parents, Yusra and Jamil Rababa. My parents taught me the value of hard work and dedication. My parents provided endless and generous Support to help me accomplish this goal.

I also want to thank my Wife, Ayat, and my sons, Hashim and Zain for generous support and patience. Through this journey, I learned that my wife is capable of so much more than I ever expected. I am thankful that I have such wife who always stands with me during all times of personal hardships. 


\section{TABLE OF CONTENTS}

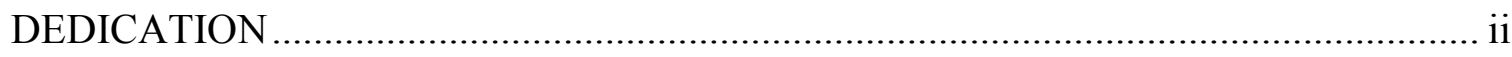

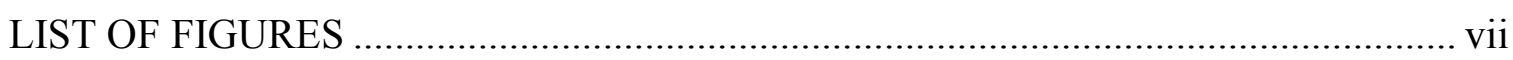

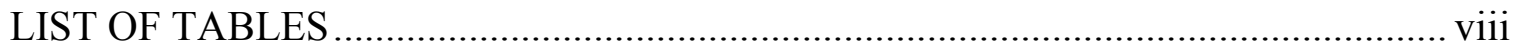

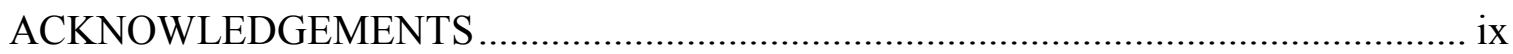

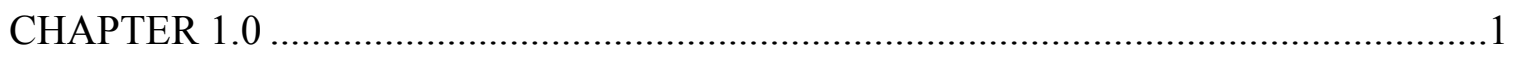

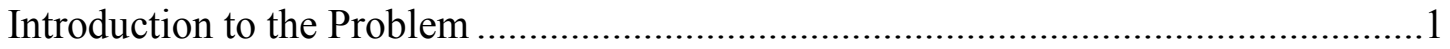

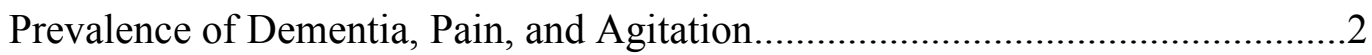

Critical Thinking Problems in Care of Pain in PWD ..................................................

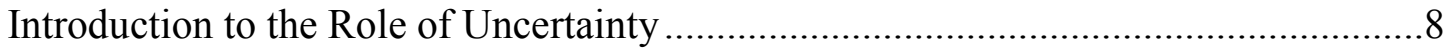

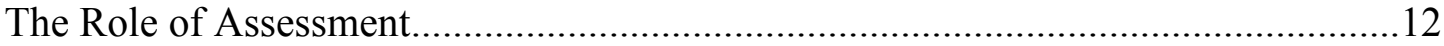

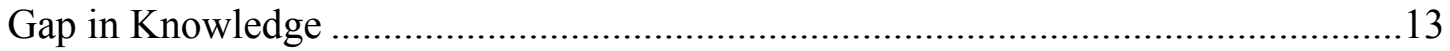

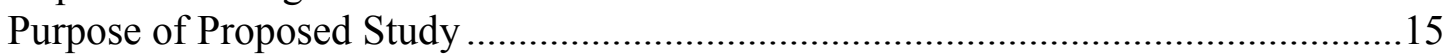

Research Questions and Hypotheses .................................................................16

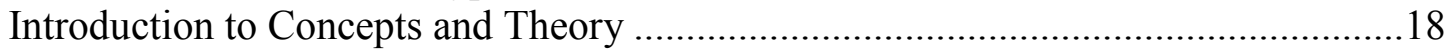

Responses to Certainty of Pain Model ...............................................................18

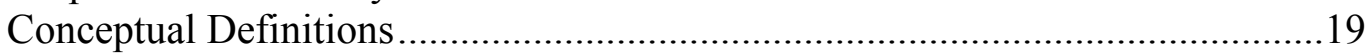

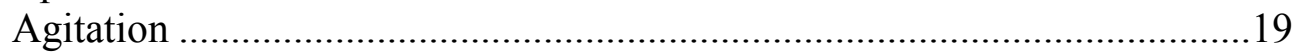

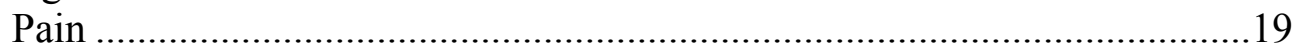

Certainty/Uncertainty Regarding Suspected Pain........................................21

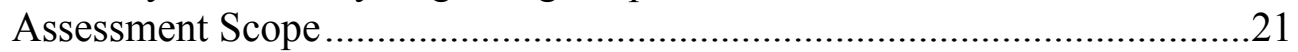

Background on Proposed Study Setting and Sample..................................................21

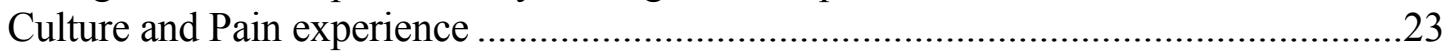

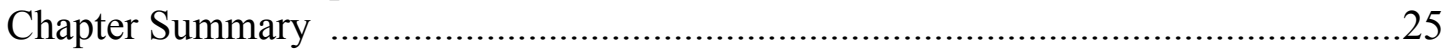

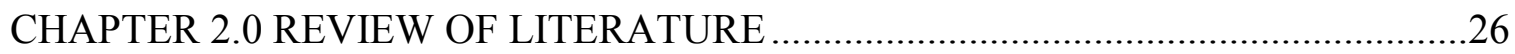

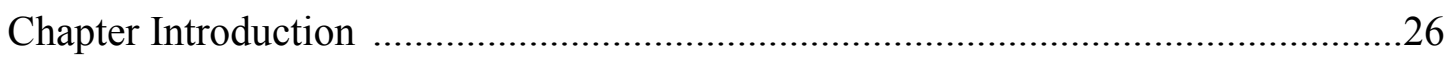

Section 2.1 - Manuscript One "Pain Assessment in Elderly with Dementia: Remaining

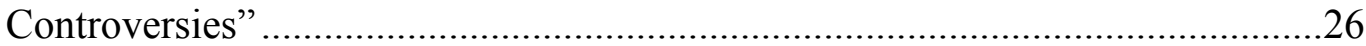

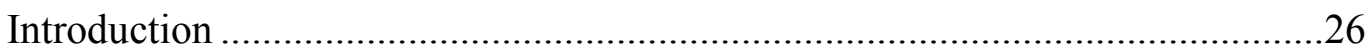

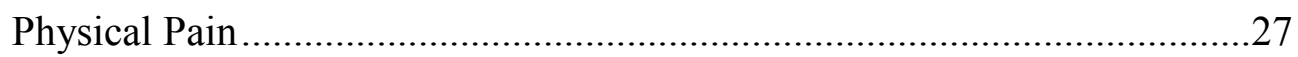

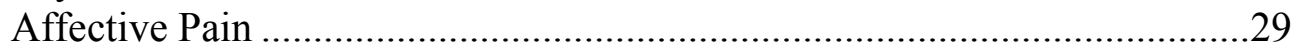

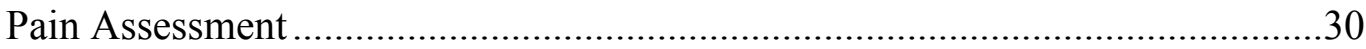

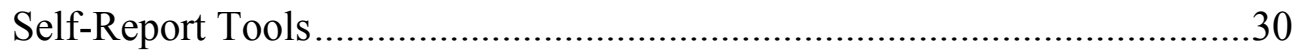

Observational Pain Tools In Dementia .........................................................

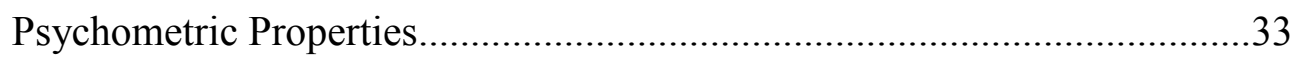

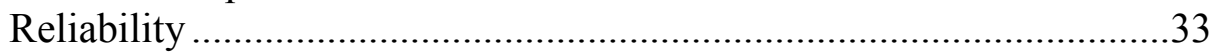

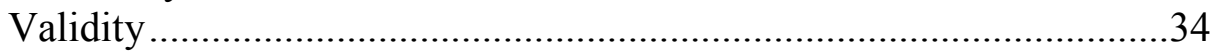

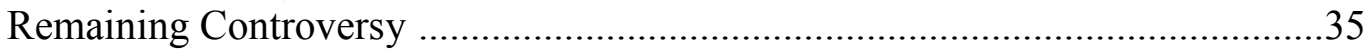


Conclusion.

Section 2.2 Manuscript 2: Does Uncertainty Explain Poor Pain Management in People

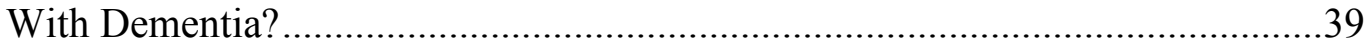

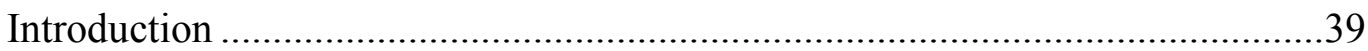

Critical Thinking and Decision Making .........................................................42

Background to Decision Making Theory ........................................................44

Background of Field of Healthcare Decision-Making ............................44

Overview of Core Nurse Decision-Making Models ...............................44

The Cognitive Continuum Theory (CCT) .....................................45

Adaptive Pain Management (APM) ................................................46

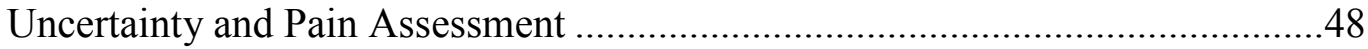

Introduction to the Response to Certainty of Pain model (RCP) ...............48

Nurses' Certainty Trajectory.............................................................50

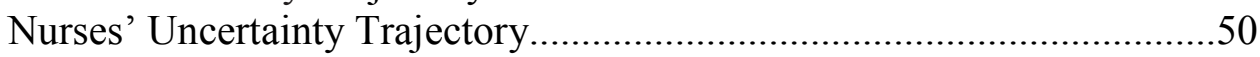

Other Theoretical References ...............................................................51

Evidence That Relates to Uncertainty and Pain Treatment .....................53

Future Research and Further Theoretical Development .................................54

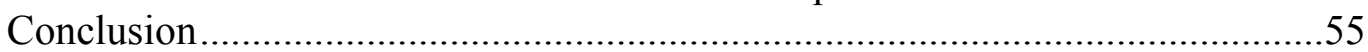

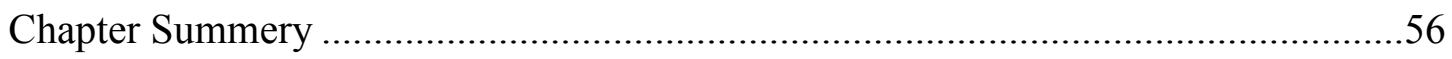

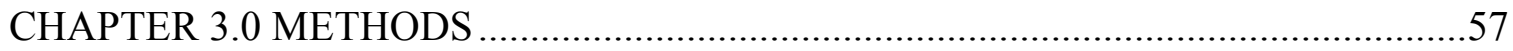

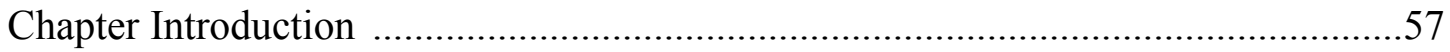

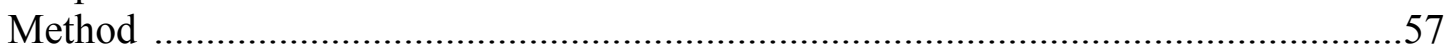

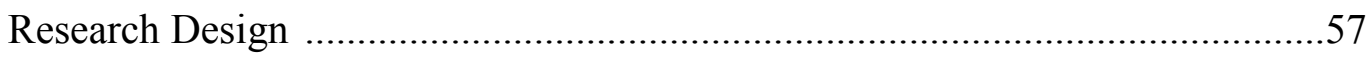

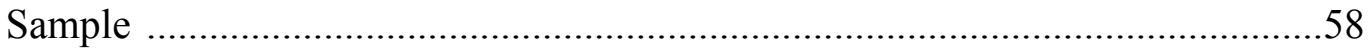

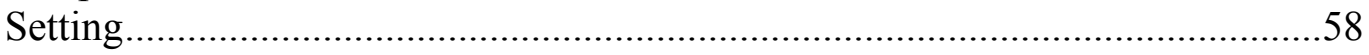

Instruments and Operational Definitions ..............................................6 60

Outcome Instruments ...............................................................60

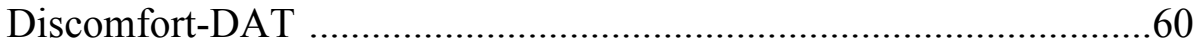

Wisconsin Agitation Inventory ..............................................61

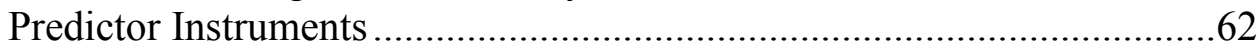

Nurses' Certainty .....................................................................62

Assessment Scope .................................................................62

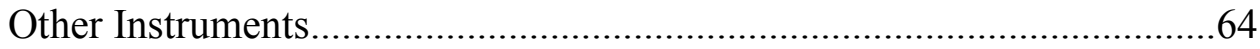

Mini Mental Status Exam ..........................................................64

Cumulative Illness Rating Scale for Geriatrics...............................64

Communication of Need - Dementia Alzheimer's Type ..................64

Human Subjects Considerations..............................................................6

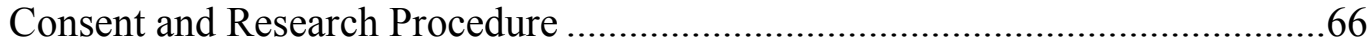

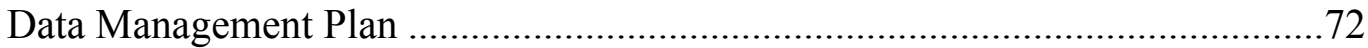

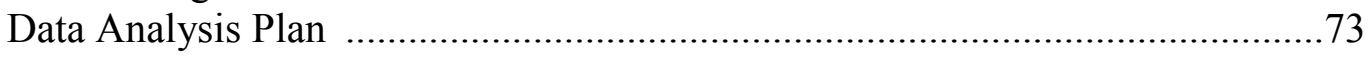

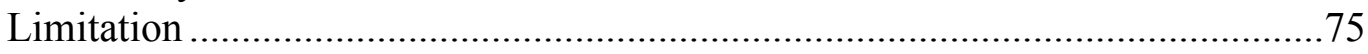

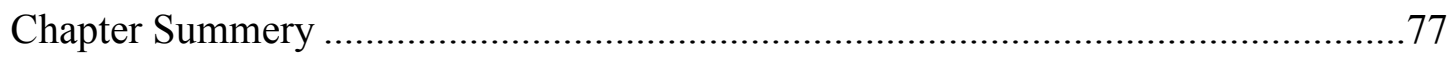

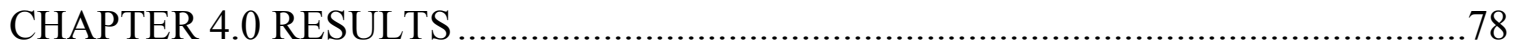


Section 4.1 - Manuscript Three "The Association of Nurses' Assessment and Certainty to

Pain Management and Outcomes for Nursing Home Residents in Jordan".........78

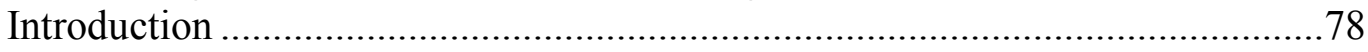

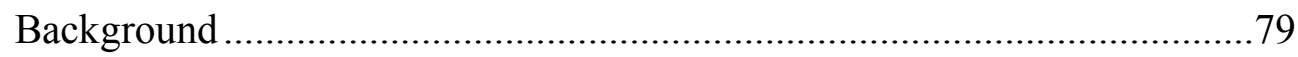

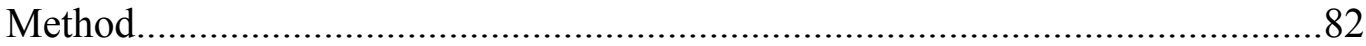

Research Design, Sample, Setting ...................................................... 82

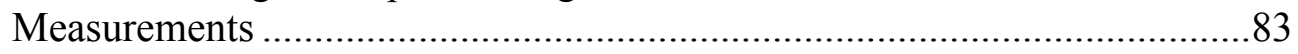

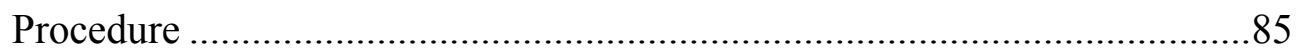

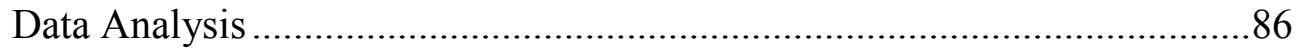

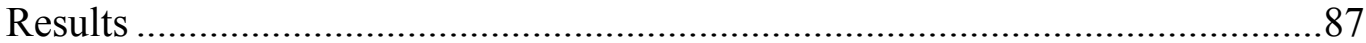

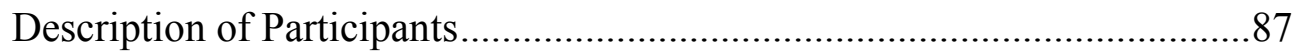

Comparisons Based on Levels of Dementia and Communication Deficit ...87

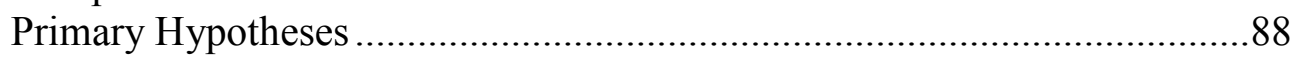

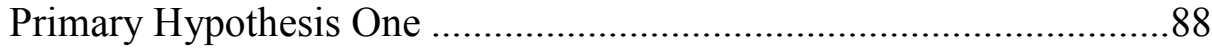

Primary Hypothesis Two ..............................................................88

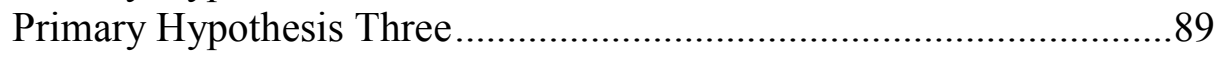

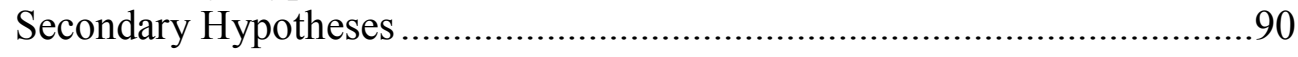

Secondary Hypotheses One .........................................................90

Secondary Hypotheses Two .......................................................... 91

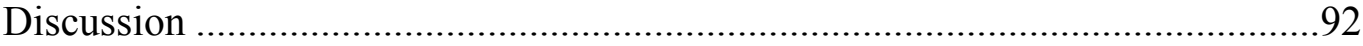

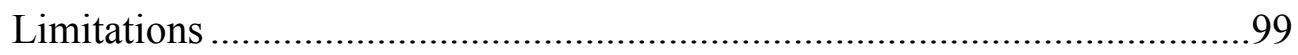

Implications and Future Research.................................................102

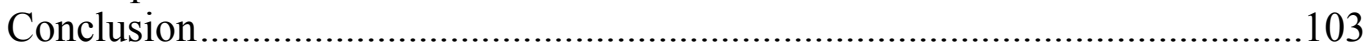

Section 4.2 Study Report "The Association of Comorbid Burden to Patients Outcomes of

Nursing Home Residents with Dementia in Jordan".......................................103

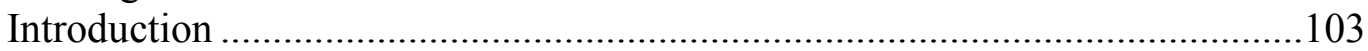

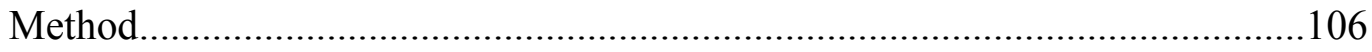

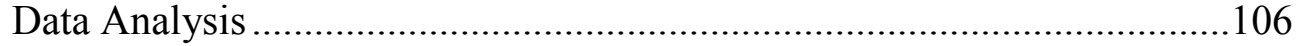

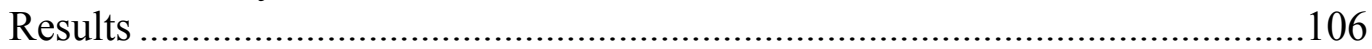

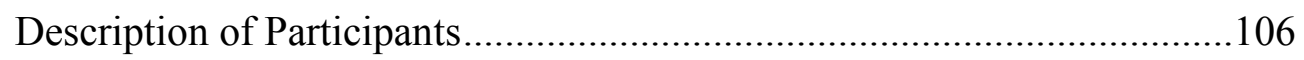

Description of Comorbid Conditions and Ability to Verbally Report Symptoms

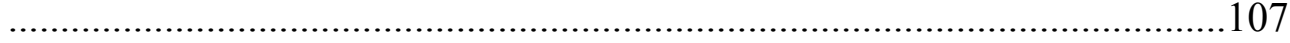

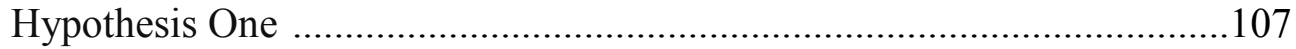

Hypothesis Two ..................................................................... 108

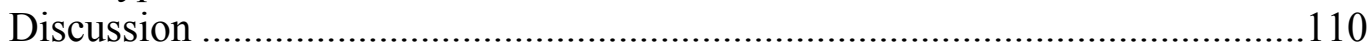

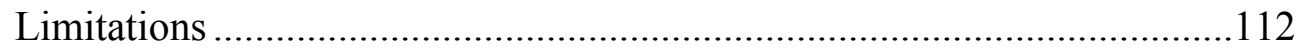

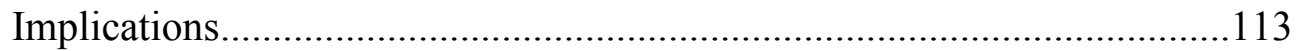

Recommendations For Future Research ..............................................113

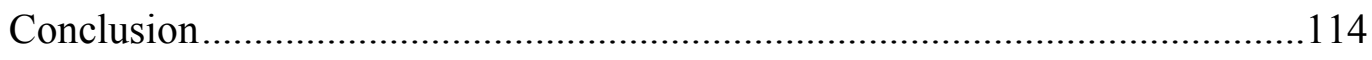

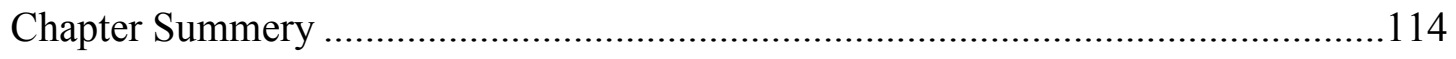

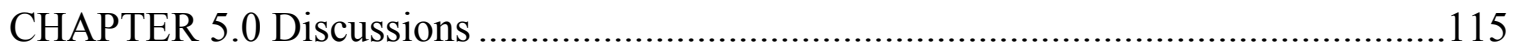




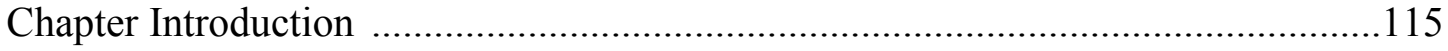

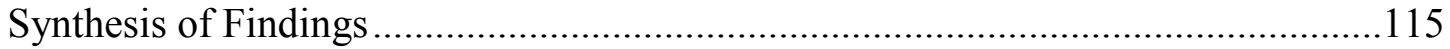

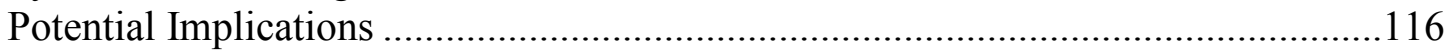

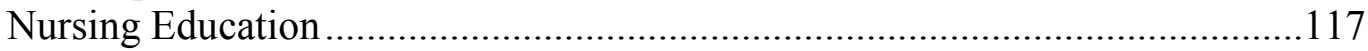

Clinical Practice in Nursing Homes ..............................................................118

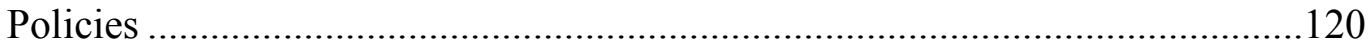

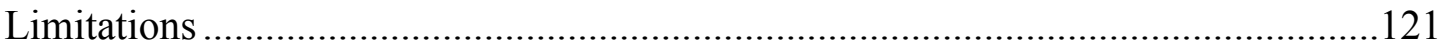

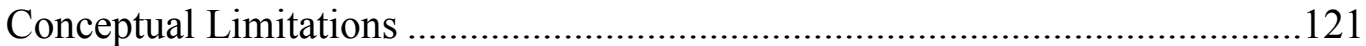

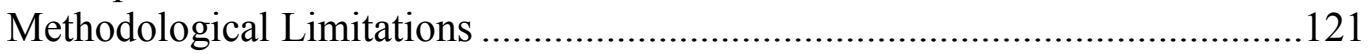

Recommendations For Future Research ..........................................................125

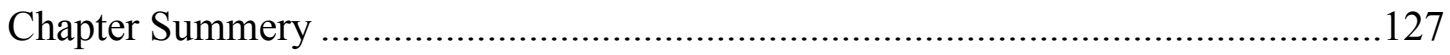

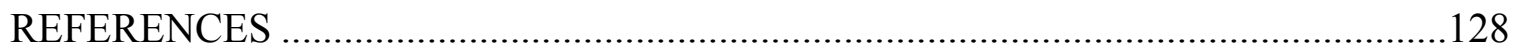

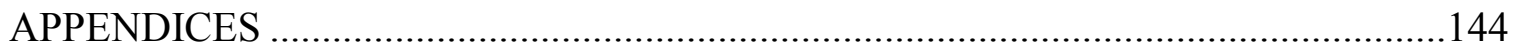

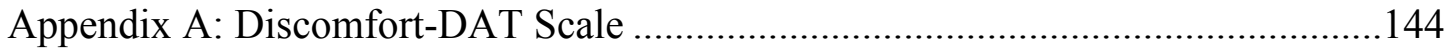

Appendix B: Wisconsin Agitation Inventory Scale ...............................................145

Appendix C: Mini Mental Status Exam Scale ………………...................................148

Appendix D: Cumulative Illness Rating Scale for Geriatrics .....................................151

Appendix E: Tracking Change Form ................................................................152

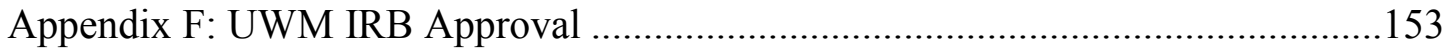

Appendix G: Resident Consent Form ..................................................................154

Appendix H: Proxy Consent Form …………………....................................158

Appendix I: Nurse Consent Form ........................................................................162

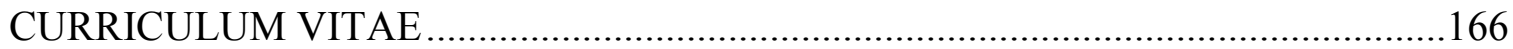




\section{LIST OF FIGURES}

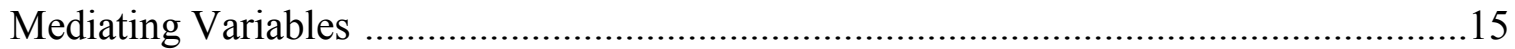

Theoretical Framework: Response to Pain Certainty Model ........................................49

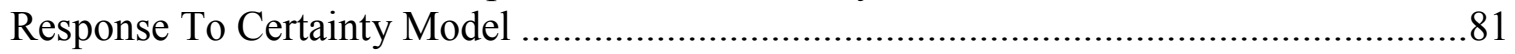




\section{LIST OF TABLES}

Summary of On-Site Consent and Research Procedures .............................................66

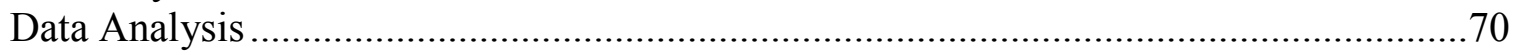

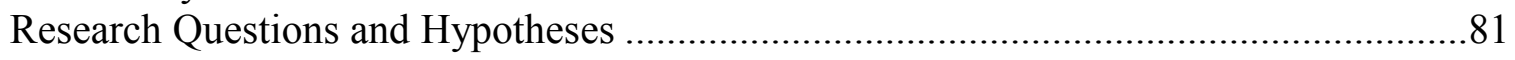

Primary Hypothesis 2: Hierarchical Regression Analysis with Agitation.......................89

Primary Hypothesis 2: Hierarchical Regression Analysis with Pain1 ............................89

Primary Hypothesis 2: Hierarchical Regression Analysis with Pain2 ............................89

Primary Hypothesis 3: Hierarchical Regression Analysis with Agitation........................90

Primary Hypothesis 3: Hierarchical Regression Analysis with Pain 1 ............................90

Primary Hypothesis 3: Hierarchical Regression Analysis with Pain2 ............................90

Secondary Hypothesis 1: Hierarchical Regression Analysis with Agitation....................91

Secondary Hypothesis 1: Hierarchical Regression Analysis with Pain 1 .........................90

Secondary Hypothesis 1: Hierarchical Regression Analysis with Pain2 .........................91

Secondary Hypothesis 2: Hierarchical Regression Analysis with Agitation....................91

Secondary Hypothesis 2: Hierarchical Regression Analysis with Pain 1 .........................92

Secondary Hypothesis 2: Hierarchical Regression Analysis with Pain2 ........................92

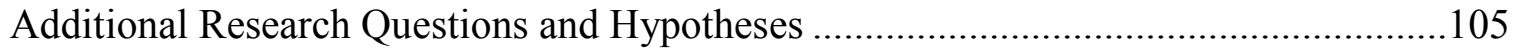

Additional Hypothesis 1: Hierarchical Regression Analysis with Agitation ..................107

Additional Hypothesis 1: Hierarchical Regression Analysis with Pain1 .......................108

Additional Hypothesis 1: Hierarchical Regression Analysis with Pain2 .......................108

Additional Hypothesis 2: Hierarchical Regression Analysis with Agitation .................109

Additional Hypothesis 2: Hierarchical Regression Analysis with Pain1 .......................109

Additional Hypothesis 2: Hierarchical Regression Analysis with Pain2 ......................109 


\section{ACKNOWLEDGEMENTS}

I would like to express my deep acknowledgments and appreciation to my major professor Dr. Christine Kovach for her generous support, guidance, and patience. Dr. Kovach has contributed a lot to my personal and academic life. At the personal level, Dr. Kovach is a special role model of the polite and humble teacher who has always treated me as a colleague. Dr. Kovach's positive attitude has fostered a memorable experience of personal growth. On the academic level, Dr. Kovach was a role model of a professional researcher who appreciates the interdisciplinary scholarly work. Dr. Kovach's openness to new ideas and different research methodologies has enriched my research experience over the years I have been one of her students.

I would like to thank my committee members: Dr. Kim Litwak, Dr. Jung Kwak, and Dr. Ellis for serving on my dissertation committee. Their contributions throughout this work have positively influenced the quality of this dissertation. I am grateful for their support and encouragement.

Finally, I would like to express my deep gratitude to my family for their generous endless support and patience. 


\section{CHAPTER 1}

This chapter begins by describing the significance of the problem of poor pain management in people with dementia (PWD). The prevalence of pain and agitation, a consequence of poorly managed pain, is provided. Next, the chapter describes in detail the process of nurse critical thinking relative to pain management for PWD. The role of nurse uncertainty regarding suspected pain is introduced and will be expanded upon in Chapter 2. Gaps in knowledge provide the context for research questions and hypotheses. Conceptual definitions are included. Since this study will be conducted in Jordan, salient cultural factors and available evidence are described.

\section{Introduction to the Problem}

Pain in people with dementia (PWD) is under recognized, underestimated, and undertreated (Lukas et al., 2012). Multiple studies have found that it is clinically challenging to assess pain in non-verbal adults. PWD often have both a cognitive impairment and aphasia that interferes with their ability to communicate effectively and clearly with their caregivers or healthcare providers (Brecher \& West, 2014; Hadjistavropoulos et al., 2007; Horgas \& Miller, 2008; Lints-Martindale, Hadjistavropoulos, Lix, \& Thorpe, 2012; Kovach, Noonan, Griffie, Muchka, \& Weissman, 2001; Kovach, Griffie, Muchka, Noonan, \& Weissman, 2000). These barriers to pain assessment in PWD may lead to misunderstanding the needs of PWD or misinterpreting their health-seeking behaviors (Horgas \& Miller). However, it is important for nurses caring for older adults with dementia to ensure they receive effective pain management, as unrelieved pain in PWD can have many serious consequences (Horgas, 2003).

Unrelieved pain in PWD can lead to agitation and a decline in physical function, diminished appetite, irritability, reduced participation in social activities, depression, delay in 
healing, sleep and activity pattern disturbance, functional and cognitive impairment, quality of life reduction, prolonged hospitalization (Horgas \& Miller, 2008) impaired nutrition, decreased mobility, and decreased immune function (Kovach et al., 2001). The 6-month mortality rate for advanced dementia is about $25 \%$ and the estimated time to live is 1.3 years with distressing pain symptoms (Manfredi, Breuer, Wallenstein, Stegmann, Bottomley, \& Libow, 2003). One of the main negative consequences of under assessed and undertreated pain is agitation (Horgas \& Miller). Pain in PWD who have aphasia and cognitive impairment may be manifested as agitation. Because studies on assessing and treating pain in people with dementia have used both pain and agitation as clinically significant outcomes, both pain and agitation are dependent variables for the proposed study (Husebo et al., 2011; Kovach et al., 2012).

Agitation is associated with many negative consequences on older adults health including depression, aggressive behavior, physiological changes such as weight loss and dehydration, institutionalization, changes in activities of daily living (ADLs), social life changes, decreased quality of life and isolation (Boult \& Wieland, 2010; Cohen-Mansfield \& Billig, 1986; Kovach et al., 2001; Kong, 2005; Dewing, 2010, Bankole et al., 2012). Agitation is associated with increased confusion in NH residents and is a burden to both family members and care providers. Agitation is one of the most commonly reported symptoms of Alzheimer's dementia (Manfredi et al., 2003).

\section{Prevalence of Dementia, Pain, and Agitation}

The proposed study took place in the country of Jordan. In most countries of the Middle East, older adults are about $5-8 \%$ of the population. Even though the number of older adults is going to multiply over the coming ten years, this demographic shift has been given little attention by health-policy makers and health researchers (Almomani, McDowd, Bani-Issa, \& Almomani, 
2014). In Jordan, $6.3 \%$ of the total population in 2010 was older adults (Almomani et al., 2014). Increased life expectancy with parallel increases in the percentage of older adults creates an imperative for nursing researchers to pay more attention to the quality of life of nursing home residents. Nevertheless, there is evidence that in Arab countries pain in nursing home residents with dementia is still under assessed and undertreated (Almomani et al.).

Pain is one of the most prevalent problems in older adults, as approximately $80 \%$ of those living in nursing homes and up to 50\% living at home suffer pain (Lukas et al., 2012). According to Lukas et al. pain is one of the most common chronic symptoms distressing older adults with advanced dementia. According to Husebo et al. (2011), pain affects $50 \%$ to $80 \%$ of NH residents with dementia. Several studies have consistently shown the high prevalence of poor assessment and delayed management of pain in NH residents with dementia (Reynolds, Hanson, DeVellis, Henderson, \& Steinhauser, 2008; Williams, Zimmerman, Sloane, \& Reed, 2005; Wu, Miller, Lapane, Roy, \& Mor, 2005). Many studies have shown that the number of older adults with pain is more than the number of youth with pain. Eighty-six percent of noninstitutionalized rural older adults had had pain at some time in the previous year, and 59\% had had multiple pain complaints (Huffman \& Kunik, 2000).

Treatments for pain in older adults are underutilized. Approximately $75 \%$ of 97 institutionalized older adult patients had pain and only $15 \%$ of those with pain had received analgesics in the last 24 hours. Also, $65 \%$ of nursing home residents had chronic pain and less than $50 \%$ of these painful situations had been detected by their primary healthcare providers (Huffman \& Kunik, 2000). Also, According to Parker (2013), the incidence of untreated pain among nursing home residents in the USA is between $49 \%$ and $84 \%$. According to Allen (2001), $41.2 \%$ of nursing home residents in the USA have untreated pain which is defined as daily, 
moderate to severe pain that persists two to six months after it was first assessed. The literature does not have specific statistics showing the prevalence of untreated pain in nursing home residents in the Middle Eastern countries.

According to Husebo, Ballard, and Aarsland (2011), 35 million people worldwide had dementia in 2011, and this number is projected to be 115 million by 2050. As of 2013, there were approximately 44.4 million PWD worldwide. It is projected that in 2030, around 75.6 million older adults will have dementia. Cervo et al. (2007) reported that more than 4 to 5 million people are affected with dementia in the United States. According to recent epidemiological studies more than 7 million in the USA alone are affected by dementia (A1Adawi et al., 2014). According to Al-Adawi et al., by the year 2047, the incidence of Alzheimer's dementia is projected to affect 8.64 million American people. Approximately 60 to $80 \%$ of nursing home residents have dementia (National Institute on Aging, 2006; Vicious, 2002). The projected aging of our population, along with a parallel increase in the prevalence of dementia make effective pain assessment in dementia of paramount importance. According to Bowirrat et al. (2005), the prevalence of Alzheimer's dementia is approximately $20.5 \%$ of Israeli Arab's older adults who are older than 60 years old and $60.5 \%$ of those who are older than 80 years old.

Agitation affects $40 \%$ of patients in nursing homes with dementia in the USA (Johnson \& Taylor, 2011). No study in the literature shows the prevalence of agitation among nursing home residents with dementia in Middle Eastern countries. There certainly is no study in the literature shows the prevalence of agitation among nursing home residents with dementia in Jordan. 


\section{Critical Thinking Problems in Care of Pain in PWD}

\section{Introduction}

Critical thinking is not a simple, automatic or linear process, but has been described as a process acquired through hard work, commitment, and an active curiosity toward learning and decision-making (Price, 2015). The process of critical thinking includes bringing knowledge into a situation, gathering information, and reflecting critically on what is already known and what has been assessed to identify a problem. Also, critical thinking is an intellectual process in which skillful reasoning is applied to guide actions (Aliyu, Mathew, Paul, Shinaba, Oyewole, \& Olusanya, 2014). Furthermore, critical thinking is outcome-focused thinking that in nursing is guided by standards, policies and procedures.

Critical thinking in nursing is viewed as a nursing process, which includes assessment and nursing diagnosis, planning, implementing nursing interventions, and evaluation. While this perspective of critical thinking may be useful for teaching and multiple clinical purposes, it fails to adequately describe the processes of critical thinking used by nurses in some clinical situations. These clinical situations are usually underdetermined, ambiguous, and complex. The nursing process fails to consider the complexity of clinical judgments that nurses face every day and the factors that impact these judgments (Tanner, 2006). Therefore, nurses using the nursing process may have difficulty defining these clinical situations, interpreting their meanings, and responding appropriately.

\section{Knowledge Deficit}

According to Achterberg et al. (2013), nurses have knowledge deficits about the presentation of illnesses in older adults. The complexity of pain assessment in PWD as well as their inability to communicate may contribute to nurses' knowledge deficit. Knowledge deficits 
affects nurses' critical thinking skills when making decisions about assessment and treatment of pain in PWD (Achterberg et al., 2013). According to Gilmore-Bykovskyi and Bowers (2013), easily accessible decision support tools that apply evidence-based guidelines for pain treatment are needed to improve assessment and treatment of pain in PWD (Gilmore-Bykovskyi \& Bowers). Yet, long-term care nurses' understanding of evidence-based pain management guidelines in older adults, particularly for those with dementia and chronic pain, is limited (Achterberg et al., 2013). Instead, nurses often use a trial and error process (Sheilds et al., 2013). This process involves administering nonpharmachological intervention and observing PWD's responses to these interventions or just looking for the reversal of behavioral changes (Sheilds et al.).

\section{Misconceptions}

Achterberg et al. (2013) found that nurses held numerous misconceptions regarding pain in older adults, which presented problems to their critical thinking. For example, a recent study showed that nurses' own beliefs about a patient, instead of objective data, affected their critical thinking about pain management. These beliefs and attitudes arise from common misconceptions about pain in older adults, such as the following: (a) pain is a normal part of aging; (b) aging cause pain insensitivity; (c) PWD do not feel pain; (d) analgesics have dangerous side effects if prescribed for older adults; or (e) the lack of self-reporting of pain means no pain (Achterberg et al.).

\section{Intuition}

According to the model of critical thinking processes proposed by Papp et al. (2014), critical thinkers use analytical strategies to assess a problem. They adapt their thinking as is applicable to the context, congruent with fundamental principles and concepts, and avoid 
guessing. Also based on the model, critical thinkers understand complex connections between concepts to create reasonable hypotheses to explain observed phenomena (Papp et al.). Accordingly, when nurses are able to think using a logical and systematic approach with questioning and inference, they are able to apply their critical thinking skills for clinical decision-making processes (Monroe, Parish, \& Mion, 2015). However, nurses are not adequately trained to use critical thinking skills. Instead, nurses have been found to use intuitive and 'trial and error' approaches (Gilmore-Bykovskyi \& Bowers, 2013).

Intuition is defined as rapid data processing that occurs with low conscious awareness (Cader, Campbell, \& Watson, 2005). For example, nurses may report that they just feel inside something is wrong because the patient is not being herself. In this example, nurses' intuition is not enough to inform their critical thinking of the suspected clinical problem (Shega, Hougham, Stocking, CoxDHayley, \& Sachs, 2006).

According to Gilmore-Bykovskyi and Bowers (2013), nurses' uncertainty is the major reason that nurses tend to use their intuition when assessing pain in PWD. Nurses use their intuition to assess pain in PWD because they are uncertain if the behavioral changes they observe represent pain. Also, nurses do not have a clear understanding of decision-making processes and other relevant information (Gilmore-Bykovskyi \& Bowers). Therefore, nurses not only cannot recognize the behavioral changes in PWD but they are also unable to clearly translate their intuition into meaningful data, facilitating clinical decisions about pain (Shega et al., 2006). Critical thinking in the care of pain in PWD is based on intuition in most cases (Kaasalainen et al.). Although critical thinking is essential to a nurse's ability to assess and treat pain in PWD, it remains a challenge to measure (Kaasalainen et al.). 


\section{Bias}

Critical thinking is more impacted by what nurses brings to clinical situations than the recorded assessment about the situations (Tanner, 2006). According to Tanner, nurses come to clinical situations with biases that strongly influence their critical thinking and decisions. When making a clinical pain assessment based on observation, it is important to be aware of biases that may confound nurses' critical thinking (Horgas \& Miller, 2008). According to Mahoney et al. (2008), nurses' critical thinking is the product of both bottom-up input, and top-down processing, wherein nurses' experience and biases may impact upon the critical thinking. Many recent studies indicate that nurses are quite sensitive to pain expression when identifying its presence or the difference between broad categories of pain expression (Cervo et al., 2007; Fuchs-Lacelle, et al., 2004; Hadjistavropoulos et al., 2012; Horgas et al., 2007; Lin et al., 2011; Zwakhalen et al., 2012).

Nevertheless, nurses display substantial biases in their critical thinking of patients' pain. According to Chen et al. (2010), nurses consistently underestimate the pain of others. This bias seems to vary with several factors including race, likability, and perceptions regarding motivations (Fuchs-Lacelle et al., 2004). The underestimation bias is higher among nurses with more clinical experience with pain because of overexposure to evidence of pain in others (Fuchs-Lacelle et al.; Zwakhalen et al., 2012).

\section{Introduction to the Role of Uncertainty}

Uncertainty regarding suspected pain may be a major barrier to timely and effective pain assessment and management in both Western and Middle Eastern nursing homes (Almomani et al., 2014: Gilmore-Bykovskyi \& Bowers, 2013). Nurses' identification of pain symptoms in NH residents with dementia depends mainly on the presentation of behavior changes such as 
withdrawal, restless behaviors, and negative vocalizations. Signs and symptoms of pain in PWD are sometimes referred to as health seeking behaviors (Horgas \& Miller). These behavior changes are still under-recognized by nursing home nurses. Since PWD are unable to communicate their need effectively, health care providers may misunderstand or misinterpret their patients' health seeking behavior (Horgas \& Miller).

Health-seeking behaviors of PWD represent an inability to make needs known and for caregivers to comprehend needs. These behaviors include negative vocalization, irritable body movement, grimacing, guarding position, aggressive behaviors, nonverbal vocal complaining, rigidity, scratching knees, and meaningless movement (Feldt, 2000). However, nurses do not have a clear understanding of how the behavior changes in PWD are manifested, even in those PWD who could self-report their pain. Inconsistency of behavioral changes associated with pain in PWD may contribute to nurses' uncertainty. Also, it is often unclear whether pain or another unmet need is the primary cause of these behavior changes.

As a result, even though nurses identify behavioral indicators of pain in residents with dementia, they generally have very low levels of certainty regarding suspected pain for individual residents. The presence of behavioral indicators of pain is not consistently reliable enough for nurses to feel certain about starting pharmacological treatment. Rather, nurses try other interventions to relieve behavior changes. Therefore, pharmacological treatment is often delayed (Gilmore-Bykovskyi \& Bowers, 2013).

Behavioral indicators of pain are difficult for nurses to interpret because of relatively low specificity of behavioral symptoms for pain (Kovach, Logan, Joosse, \& Noonan, 2012). Behaviors such as restlessness that are indicators of pain are also commonly seen when psychological and environmental needs are present (Kovach, Noonan, Schlidt, \& Wells, 2005). 
Nurses may start by providing a trial of a nonpharmacological intervention before administering an analgesic (Kovach et al., 2012). Also, if the nurse interprets the behavior as having a psychological etiology rather than as a symptom of pain, a psychotropic medication may be inappropriately prescribed (Kaasalainen et al., 2007).

Consistently, Jordanian nurses are uncertain regarding suspected pain in older adults who have different presentations of illnesses (Eid, Manias, Bucknall, \& Almazrooa, 2014). It is hypothesized that this uncertainty is primarily caused by practice misconceptions and nurses' knowledge deficits regarding pain in PWD (Eid, et al.). According to Kovach et al. (2000), nurses receive little or no formal education about pain management in late-stage dementia. Furthermore, nurses poorly understand the current strategies of pain assessment and treatment in PWD applied in US nursing homes. Consistently, Jordan is lagging behind in gerontology practice. Healthcare institutions in Jordan do not teach gerontology and definitely pain management in PWD to nursing students. Uncertainty is perceived to be a major barrier to effective treatment of pain in NHs of Middle Eastern countries like Jordan (Eid, et al.)

Because nurses commonly use simple self-reporting assessment tools that do not capture behavioral expressions of pain, health-seeking behaviors may be difficult for the nurse to interpret and create a level of uncertainty regarding the presence or absence of pain (GilmoreBykovskyi \& Bowers, 2013). Because of the recognized complexity in understanding this underassessment and undertreatment of pain in PWD, Gilmore-Bykovskyi and Bowers developed the Responses to Certainty of Pain (RCP) model, which incorporates the concept of certainty and how it relates to the problem (Gilmore-Bykovskyi \& Bowers).

The RCP model is inductively derived from interviews with practicing nurses. It is compelling that the RCP model is the first model to posit relationships between nurses level of 
pain certainty, assessment scope and patient outcomes. Regardless of whether or not the tenets of the RCP model are or are not supported in this study, findings will provide significant information on the clinical outcomes associated with different types of assessment.

When a person with dementia has a change in behavior or condition, nurses may make various conjectures or decisions as a part of critical thinking processes (Chang, Oh, Park, Kim, \& Kil, 2011; Gilmore-Bykovskyi \& Bowers, 2013; McDonald, 2014). The RCP model describes trajectories that are hypothesized to occur when a nurse has a high and low level of certainty of suspected pain. These trajectories are outlined below.

When a nurse has a high level of certainty of suspected pain:

1. A unidimensional assessment is more likely to be done than a multidimensional assessment.

2. If the unidimensional assessment leads to a high level of certainty of pain, then treatment will be timely and the person will have better outcomes.

3. If the unidimensional assessment leads to a low level of certainty of pain, then treatment will not be timely and the person will have worse outcomes.

When a nurse has a low level of certainty of suspected pain:

1. The nurse may respond by doing a multidimensional assessment or by using trial and error approach to managing the change in condition.

2. If the nurse uses a multidimensional assessment of pain, she/he is more likely to have a high degree of certainty regarding whether pain is present and the PWD is more likely to have better outcomes.

3. If the nurse uses a trial and error approach to managing the change in condition, then the PWD is more likely to have worse outcomes. 


\section{The Role of Assessment}

Nurses are taught that self-report of pain is the gold standard (Kovach, Noonan, Griffie, Muchka, \& Weissman, 2001) and that pain is "a negative emotional and/or physical state subject to variation in magnitude in response to internal or environmental conditions" (Hurley, Volicer, Hanrahan, Houde, \& Volicer, 1992, p. 374). The observations of pain behaviors in PWD vary considerably depending on many factors. These factors include observer bias, the presence of others during the observation, the activity status of the person being observed, and the timing of measures. Therefore, nurses usually prefer to use self-report tools to assess pain in people with mild dementia (Hadjistavropoulos et al. 2007). However, Scherder et al. (2005) indicate that most self-report tools do not measure the behavioral symptoms of pain but intensity. Therefore, both self-report and observational tools would be used together to measure both aspects of pain in PWD (Huffman, \& Kunik, 2000; Scherder et al.).

Scientists in the field postulate, and there is some beginning evidence supporting that, there is a decrease in the affective component of pain in people with dementia (Kovach, 2013; Rudich, Lerman, Gurevich, \& Shahar, 2010; Wilson, Uhelski, \& Fuchs, 2008). Since self-report tools capture affective components of the pain experience, people with dementia are likely to under-report pain if self-report is the only measure used. Observational measures are more likely to capture behavior changes derived from the physiological component of pain rather than the affective component (Kovach, 2013).

The Unites States has seen a plethora of research in the past 20 years on improving assessment of pain in people with dementia (Dening, 2014; Hadjistavropoulos, Herr, Prkachin, Craig, Gibson, Lukas, \& Smith, 2014; Lichtner et al., 2014; McIlfatrick, 2015; Zwakhalen, Hof, \& Hamers, 2012). Multiple assessment tools have been developed that assess behavioral 
symptoms of pain in people with dementia (Ali et al., 2015; Ersek, Herr, Neradilek, Buck, \& Black, 2010; James, 2011; Knebel, Haberstroh, Kümmel, Pantel, Schröder, 2015). Kovach et al (2012) have consistently found that improving nurse's assessment of behavioral symptoms leads to decreased pain and agitation in PWD (Kovach et al., 2006; Kovach et al., 2010; Kovach et al., 2012). However, the American Geriatrics Society Expert Panel (Boyd et al., 2012) still suggests that unidimensional assessment may be all that is needed if the person can self-report.

\section{Gap in Knowledge}

Even though the literature describes the complexity of pain assessment and management in PWD, no study has directly examined the relationships between nurse's certainty regarding a patient's pain, the complexity of their pain assessment, and patient outcomes. In addition, experts in the field have provided contradictory advice on the preferred methods of pain assessment, particularly for those with dementia who can still verbally report some symptoms.

A growing body of literature has linked nurse uncertainty to poor pain management (Achterberg et al., 2013; Gilmore-Bykovskyi \& Bowers, 2013; Kaasalainen et al., 2007; Sheilds et al., 2013 Shega, Hougham, Stocking, CoxDHayley, \& Sachs, 2006; Tait et al., 2009).

However, only the RCP models talks specifically about nurses' uncertainty regarding suspected pain in PWD. To date this model empirically has never been tested. There is a lack of testing of the Responses to Certainty of Pain (RCP) model in nursing home residents with dementia having pain in general, and specifically in Middle Eastern countries. Also, no study has described the relationship between nurses' certainty, assessment scope, and pain outcomes in Jordanian nursing home residents with dementia. The study tested the RCP model in a sample of Jordanian nursing home residents having pain. 
The RCP model provides an explanation of how the level of nurses' certainty may predict the level of pain and agitation in nursing home residents with dementia. The RCP model suggests that when nurses are certain about pain in PWD they just need to do brief self-report assessment (i.e. Unidimensional) and then administer an analgesic in a timely manner to treat pain and agitation. The RCP model proposed that when there is certainty, there is no need to use deep analytical thought processes and using a brief self-report assessment of pain is sufficient. When nurses are not certain about pain they need to do additional assessment (multidimensional) and then the treatment of pain and agitation may be delayed (Gilmore-Bykovskyi \& Bowers, 2013). When there is uncertainty, a multidimensional pain assessment is needed to facilitate the nurses' use of deeper analytical thought processes (Gilmore-Bykovskyi \& Bowers). Another tenet of the RCP model is that nurse's over reliance on self-report (i.e.unidimensional assessment) of pain in people with dementia when uncertainty is present contributes to uncertainty and poor outcomes. A multidimensional assessment may or may not utilize selfreport, but also involves assessing for behavioral and functional changes (Gilmore-Bykovskyi \& Bowers, 2013). These tenets have not been empirically tested.

Mediating variables are critically important in understanding many complex human health phenomena and health care delivery (Bennett, 2000). A mediator has been described as a variable that specifies how the association occurs between an independent variable and a dependent variable (Bennett, 2000). According to Gilmore-Bykovskyi and Bowers (2013), it is hypothesized that a mediator, post-assessment level of certainty explains the relationship between the assessment scope and level of pain and agitation in PWD (figure 3, A). It is clearly stated in the RCP model how a significant direct effect could exist between the mediator (postassessment level of certainty) and the outcome variable, level of pain and agitation in PWD. 
According to the RCP model, pain and agitation will be lower in PWD who receive a unidimensional assessment from nurses who report a high level of certainty of suspected pain after assessment compared to PWD who receive a unidimensional assessment from nurses who report a low level of certainty after assessment (Gilmore-Bykovskyi \& Bowers).

This study also tests mediating effect of assessment scope on the relationship between pre-assessment level of nurses' certainty and outcomes, which is somewhat contrary to the RCP model (Figure 3, B). Hypothesizing that it is assessment scope regardless of post-assessment level of certainty that drives outcomes, this study attempts to contribute to the extant literature by describing the relationship between pre-assessment level of nurses' certainty, assessment scope, and the level of pain and agitation in PWD.

Figure 1: Mediating Variables

(A)

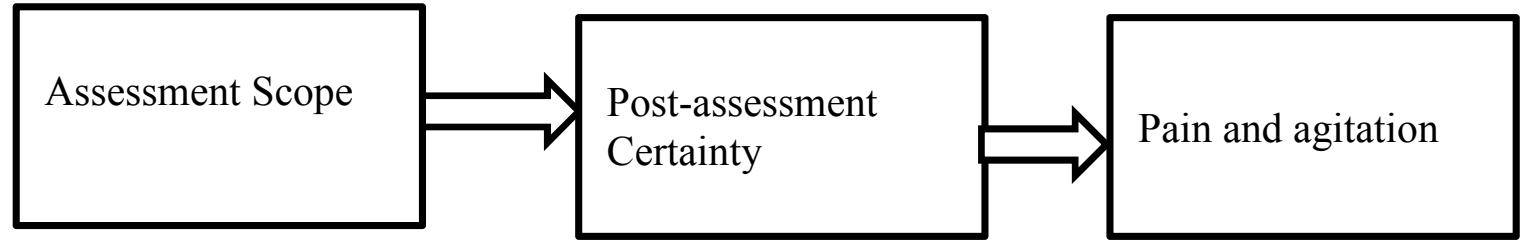

(B)

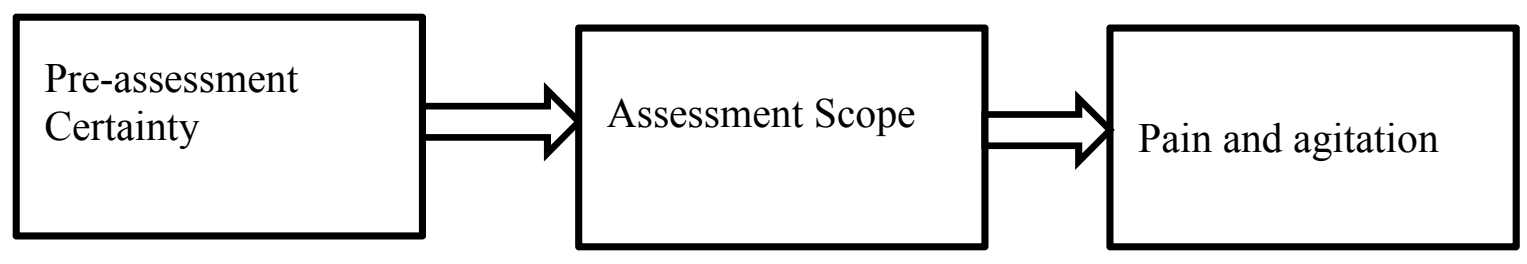

This study has the potential to bring comfort to an often neglected and vulnerable population by making a contribution to redesigning the delivery of pain assessment and management protocols applied in nursing homes in Jordan and in other Middle Eastern countries.

\section{Purpose of Proposed Study}

This study is based on the premise nurses' uncertainty regarding suspected pain or the 
presence of pain in PWD contributes to unrelieved pain in PWD (Kovach Logan, Joosse, \& Noonan, 2012b; McCaffery, Ferrell, \& Pasero, 2000). Accordingly, the purpose of this study is to examine temporally based relationships between change in behavior, the nurses' level of certainty regarding pain, assessment scope and outcomes of pain and agitation.

\section{Hypotheses and Research Questions}

The research questions that this study will address are as the following:

(1) What is the level of nurses' certainty about suspected pain in nursing home residents with dementia in Jordan before and after assessment?

(2) Is there a difference in nurses' certainty of suspected pain between residents with mild, moderate and severe dementia?

(3) Is there a difference in nurses' certainty of suspected pain in residents who do and do not verbally report symptoms?

(4) What is the frequency of using unidimensional and multidimensional assessment?

(5) Is there a difference in nurses' use of unidimensional and multidimensional assessment between residents with mild, moderate and severe dementia?

(6) Is there a difference in nurses' use of unidimensional and multidimensional assessment between residents who do and do not verbally report symptoms?

The hypotheses that this study will address are as the following:

\section{Main Hypothesis}

(1) Certainty of suspected pain by the nurse will predict type of pain assessment provided to a PWD who has a change in condition.

Rationale: According to the RCP model, nurses with a high level of certainty are more likely to conduct a unidimensional rather than a multidimensional assessment. 
(2) Pre-assessment level of nurses' certainty, assessment scope and post-assessment level of certainty will predict pain and agitation outcomes.

Rationale: Step 3 of this regression model will test if, consistent with the RCP model, post assessment certainty is a unique significant predictor of resident outcomes.

(3) The relationship between pre-assessment level of nurses' certainty and outcomes is mediated by assessment scope.

Rationale: Step 3 of this regression model will test mediating effects that are somewhat contrary to the RCP model by hypothesizing that it is assessment scope regardless of level of certainty that drives outcomes.

\section{Subgroup Hypothesis}

Hypothesis deduced from model when certainty is initially high.

1. The relationship between assessment scope and outcomes will be mediated by post assessment level of certainty.

Rationale: According to the RCP model, pain and agitation will be lower in PWD who receive a unidimensional assessment from nurses who report a high level of certainty of suspected pain after assessment compared to PWD who receive a unidimensional assessment from nurses who report a low level of certainty after assessment.

Hypothesis deduced from model when certainty is initially low

2. Assessment scope and post-assessment certainty will predict outcomes.

Rationale: According to the RCP model, pain and agitation will be lower in PWD who receive a multidimensional rather than a unidimensional assessment from nurses who report an initial high level of uncertainty of suspected pain before assessment. 
Also the RCP model predicts that a high level of post-assessment certainty predicts outcomes.

\section{Introduction to Concepts and Theory}

\section{Responses to Certainty of Pain Model}

The literature has shown that certainty has many positive outcomes in nursing decisionmaking processes (Cader, Campbell, \&Watson, 2005). According to Cader, Campbell, and Watson, certainty increases the consistency of assessment among care providers and facilitates communication and evaluation of clinical decisions. In order to improve outcomes, Nurses have recognized that they would clearly understand the thinking processes that inform nurses' actions (Kaasalainen et al., 2007).

A whole host of research has been done on nursing decision-making certainty and different terms have been used including clinical reasoning, critical thinking, and decisionmaking. These various models all come from perspectives that are based on analytical thought processes (Lauri \& Salentara, 2002). Analytical thought process has been defined as a process of problem solving that involves separation of a whole into its constituent parts in order to study the parts and the relations between parts (Hightower, 2014). Many recent studies confirm the advantage of analytical thought process over simple information processing (Hightower). Simple information processing is quite insufficient because of the complexity and uncertainty of pain in people with dementia (Leron \& Hazzan, 2009). For this study, the RCP model has been chosen because it relates directly to treatment of older adults with dementia who have difficulty communicating their pain (Gilmore-Bykovskyi \& Bowers).

The RCP model emphasized that pain is a common health problem among older adults. The model showed that nursing home residents with dementia are at high risk for 
underestimating their pain because they are unable to clearly articulate their needs. It also showed that uncertainty about pain and aging, and underuse of appropriate assessment impede evaluation of clinical decisions and pain management in $\mathrm{NH}$ resident with dementia. The model aims to explain how nurses make decisions to treat pain in nursing home $(\mathrm{NH})$ residents with dementia and to determine the situations that affect treatment decisions as well as to develop a conceptual model that can guide future research and clinical practice (Gilmore-Bykovskyi \& Bowers, 2013).

\section{Conceptual Definitions}

There are two types of definitions; conceptual and operational. Conceptual definition is the theoretical meaning of a concept and how researchers, theorists, or scientists define this concept. This section provides conceptual definitions and operational definitions will be included in Chapter 3. Researchers conducting studies need to specify conceptual definition used in their studies (Polit \& Beck, 2012).

Agitation. Agitation is defined as a response to either internal or external stimuli or both, described by older adults behavioral or verbal disruptiveness, inappropriateness and aggressiveness, which varies in degree depending on the older adults individualized thresholds (Kolanowsk et al., 2011).

Pain. For this study pain is defined as: "A negative emotional and/or physical state subject to variation in magnitude in response to internal or environmental conditions" (Hurley et al., 1992, p. 374). Pain is a sensation that is associated with actual or potential tissue damage and mediated by specific nerve fibers. The affective dimension of pain is made up of feelings of unpleasantness and emotions associated with future implications such as distress or fear (Clark, Ferrer-Brechner, Janal, Carroll, \& Yang, 1989). The sensory dimension encompasses the 
perception of the location, intensity, and quality of pain (Kunz, Lautenbacher, LeBlanc, \& Rainville, 2012).

As shown above pain and agitation are totally different concepts. The origin of pain is physiological but the origin of agitation is behavioral (Woods \& Moniz-Cook, 2012). Agitation in PWD is not an inevitable part of dementia but a sign of pain that requires treatment (Husebo, Ballard, \& Aarsland, 2011). Agitation in PWD includes many disruptive behaviors, such as resisting care, calling out, perseverant rocking, fidgeting, or striking others. Agitation in PWD is associated with cognitive impairment, which leads to increased confusion and susceptibility to overstimulation from external stimuli (Husebo et al., 2011).

PWD may easily get agitated by any degree of physical discomfort or pain (Thuathail \& Welford, 2011). For example, an older adult with dementia may rock and call out because of pain associated with arthritis. These agitation behaviors are presenting pain and will go away when pain is treated (Mahoney \& Peters, 2008). Many recent studies have found that pain can lead to increased agitation and that effective and timely pain management can reduce agitation (Husebo, Ballard, Cohen-Mansfield, Seifert, \& Aarsland, 2014; Husebo, Ballard, Sandvik, Nilsen, \& Aarsland, 2011; Sampson, White, Lord, Leurent, Vickerstaff, Scott, \& Jones, 2015). Pain behaviors can be individual and hard to differentiate from agitation (Zieber, Hagen, Armstrong-Esther, \& Aho, 2005). However, it could be possible to differentiate pain from agitation by comparing the current behavior of residents to their normal behaviors (Husebo et al., 2001). Nurses could build a comprehensive picture of a resident's normal behavior and functioning by daily interactions and observations, documented history, and reports from caregivers, and other professionals (Mahoney \& Peters, 2008). This information could be an individual baseline. Therefore, any sudden, unexpected, and unexplained changes in this baseline 
may indicate pain (Mahoney \& Peters).

Certainty/uncertainty regarding suspected pain. Nurses' certainty/uncertainty regarding suspected pain in PWD is the degree to which nurses feel they can be certain that pain is likely present (Gilmore-Bykovskyi \& Bowers, 2013).

Assessment scope. Assessment scope is the type of pain assessment provided to PWD along a continuum from unidimensional to multidimensional. Unidimensional assessment is limited to brief self-report scales. Multidimensional assessment may or may not utilize selfreport but also involves assessing for behavioral changes that are common signs of pain (assessed with or without movement) and functional changes (Gilmore-Bykovskyi \& Bowers, 2013).

\section{Background on Proposed Study Setting and Sample}

This study was conducted with nursing home residents who have dementia in Jordan and have painful conditions or any sign of pain. Jordan is the site for the study because studies of PWD have received little attention in Jordan. Jordanian demographic changes are moving towards longer life expectancy like most of the developing countries, and consequently the number of persons over 65 years of age is increasing with more PWD in the community and in the health services, including long term care services. However, the long term care of PWD is neglected in Jordan. There is a lack of awareness of the need to seek treatment if PWD have pain, which deprives the patient from the benefits of early and proper management of pain.

To my knowledge, this is the first study to describe Jordanian nurses' decision making certainty and pain management in nursing home residents with dementia. Also, Jordan was selected because of convenience for geographic closeness to the PI. The study used a descriptive 
correlational design because it is not feasible to randomly assign nurses to be certain or uncertain.

The statistics of the Ministry of Health of Jordan (1994) indicate that the number of older adults is growing rapidly and expected to constitute a higher percentage of the population in the next 20 or 30 years. This will promote the need for more nursing homes in this small Middle Eastern country. A recent study indicates that the age structure of the Jordan population has changed considerably since 1979 . This change has been attributed primarily to changes in fertility, mortality, and migration. The proportion of people older than 65 years has increased slightly from $2.8 \%$ in 1979 to $3.3 \%$ in 2012. In Jordan, the proportion of older adults are expected to reach 5\% by the year 2020 (Jordanian Department of Statistics, 2012).

An increase in the older adults population and a focus on economic development has shifted somewhat the direct responsibility to care for older adults from the family to society. The nursing home being studied was established to take care of older adults whose own family members were unable to care for them. The shift from agriculture to industry as a major source of income has resulted in women working outside of the home and young family members living abroad to study or to earn a living wage. While women and men go to work and boys and girls to school, the older adults stay by themselves and may experience loneliness and boredom. The very old who do not have the physical ability to perform activities of daily living also may experience a lack of physical care. Thus, when Jordanian family members could not be with and care for their older adults relatives, the need for support outside of the family was recognized (Almomani, McDowd, Bani-Issa, \& Almomani, 2014).

Jordan has 15 nursing homes, eight of them located in the capital, Amman, one located in the city of Zarqa, one located in the city of Alfhayse, and one located in the city of Irbid. 
Together, these nursing homes house approximately 900 residents, males and females, with the mean age of 60 years (Almomani et al., 2014). Nursing homes in Jordan only deliver general medical care for their residents by general physicians and pay little attention to providing palliative care for their residents. Although there is typically a great need for palliative care, it is not currently a major part of the care provided in nursing homes in Jordan (Almomani, Hamasha, Williams, \& Almomani, 2013). Quality of life of nursing home residents has become a crucial topic in nursing research. Yet, up till now, few studies have been conducted about quality of life in among nursing home residents in Jordan and very few studies have been conducted in other Arab countries (Almomani et al.).

\section{Culture and Pain Experience}

The reaction to and expression of pain is socially and culturally learned (Al-Harthy, Ohrbach, Michelotti, \& List, 2015). Culture is the conditioning influence in forming the individual's patterns of responding to and expressing pain (Lovering, 2006). There is extensive literature about the impact of culture on the assessment and treatment of pain in Western and non-Western cultural groups (Al-Harthy et al., 2015; Callister, 2003; Davidhizar \& Giger, 2004; Lasch, 2000; Wein, 2011; Weissman, Gordon, \& Bidar-Sielaff, 2004). Some studies have revealed differences in the assessment of pain based on the cultural background of nurses (Davidhizar \& Giger; Lasch ; Wein ). For example, according to Lovering, there were significant differences in nurses' attitudes about assessing pain between British and Sri Lankan cultures. Sri Lankan nurses were less likely to accept the patients' report of pain because they believed that they were more qualified and experienced to determine the existence of the patient's pain than the patients themselves. However, the U.K. nurses valued the patients' ability to determine their own level of pain (Lovering, 2006). In conclusion, many studies have found that culture 
influences the expression of and responses to pain in many western cultural groups (Flaskerud, 2015).

\section{Middle Eastern Culture}

The concept of pain has been studied from a cultural perspective in the context of Western culture (Al-Harthy et al., 2015; Callister, 2003). However, there has been little research done on cultural impact on pain within non-Western cultural groups like Middle Eastern countries. No study in the literature examined the difference in pain experience between nursing home residents with dementia in Middle Eastern and non-Middle Eastern countries. However, there are differences in pain expression between these different ethnicities, in general. For example, a study by Dawson and List (2009) found differences in pain threshold and tolerance between the Middle Eastern and Swedish cultures and between genders. Although these differences were not significant, they were more prominent between Middle Eastern and Swedish males than between Middle Eastern and Swedish females. Also, gender differences were more prominent within the Swedish than the Middle Eastern community (Dawson \&List, 2009).

Pain management has been limited in the Middle East by myth, irrationality, ignorance, stigma, and bias. However, all these barriers are not always cultural but simply barriers of education and understanding (Silbermann et al., 2012). Religion may impact the experience of pain. Islam is the dominant religion in Middle East, and Muslims believe that suffering from pain could be an opportunity to expiate personal sins. Yet, Islamic teaching encourages Muslims to seek treatment when they suffer, as Muslims believe that Allah did not create a disease but rather a medication for it (Halawa, Al-Diri, McLean, \& Darnall, 2015). The use of medications that might alter consciousness is prohibited in Islam. However, therapeutically prescribed opioids are 
permitted to take due to their necessity. Usually, patients and families accept using opioids for pain management when physicians or nurses clearly explain the rationale for their use (Silbermann et al., 2012; Halawa et al., 2015).

\section{Jordanian Culture}

No study in the literature has examined the difference in the experience of pain between Jordanian and non-Jordanian cultures. However, common thinking in Jordan is that men tend to be more endurable than women but in other countries women tend to be more endurable than men. Also, there are differences in the way the pain is expressed by male and female patients and in the way the pain is assessed by female and male nurses. According to Robinson \& Wise (2003), women had shorter tolerance times and higher pain ratings than men and female nurses rate pain intensity significantly higher than male nurses do.

\section{Chapter Summery}

This chapter introduced the problem of pain in PWD and the underassessment and delayed treatment or untreatment of pain. A theoretical model was described which informs the central premise of the study. If the primary hypothesis of this study is supported, results will be used to design the next study that will examine the effectiveness of pain management and patient outcomes when nurses' target assessment type to their level of certainty. Ultimately, the results of both studies could yield recommendations to be used by the Ministry of Health in Jordan to redesign the health care delivery and assessment protocol, while taking into consideration the factors that influence levels of pain and agitation in $\mathrm{NH}$ residents with dementia. This program of research will provide nurses with an evidence base of knowledge regarding pain in PWD in order to intervene effectively. 


\section{CHAPTER 2}

\section{Introduction to Chapter}

The purpose of this study is to examine the association between the nurses' decisionmaking certainty, assessment scope and the outcomes of agitation and pain in nursing home residents with dementia in Jordan. It is hypothesized that nurses level of uncertainty predicts the type of pain assessment used and that the pain assessment used predicts patient outcomes. Pain assessment scope is an important mediating variable in the proposed study. Also, it is hypothesized that nurses' uncertainty explains poor pain management in PWD. Nurses' uncertainty is an important predicting or independent variable in the proposed study. In Chapter 2 of this non-traditional dissertation proposal, a manuscript is presented that provides an overview of the current state of knowledge regarding pain assessment in people with dementia. Also, another manuscript is presented that provides an overview of the current state of knowledge regarding nurses' uncertainty regarding suspected pain in people with dementia.

\section{Manuscript 1: Pain Assessment in Elderly with Dementia: Remaining Controversies Introduction of Manuscript}

The gold standard of pain diagnosis is verbal self-reporting, but meaningful verbal communication is not always possible for older adults with advanced dementia (Kovach, Noonan, Griffie, Muchka, \& Weissman, 2001). According to Horgas and Miller (2008), the manifestations of pain in people with dementia (PWD) can also be assessed by observing changes in behavior, including facial expressions and other examples. However, neither selfreporting nor observation of changes in behavior are consistently interpreted accurately by healthcare providers. Underassessment of pain, or a missed diagnosis of pain, leads to unrelieved pain in PWD (Kovach, 2013). 
Pain in PWD often requires multidimensional assessment to ensure timely administration of analgesics and other treatments (Park, Castellanos-Brown, \& Belcher, 2010). Yet nurses often use assessment tools that underestimate pain because of poor understanding of the differences between physical and affective aspects of the pain experience and the assessment approaches that capture each experience in PWD (Kovach et al., 2008).

\section{Physical Pain}

Multiple regions in the brain act to process the state of pain and are able to modulate the experience of pain (Crawford, Malvin, Elaine, \& Douglas, 2001). The transmission of pain to the spinal cord and somatosensory cortex is done by specific nerve fiber types located at the peripheral sensory system (Crawford et al.). According to the gate theory, the physical aspect of pain experience generated by external stimuli is moderated at midbrain levels and spinal cord.

It is important to be aware of possible age-related or cognition-related changes in pain when considering the pain experience of older adult people and their ability to self-report pain during an assessment (Horgas et al., 2007). Research to date is, however, fraught with inconsistent and contradictory findings. Some studies reveal that pain may be a less frequent and severe symptom in older adults in a variety of acute medical complaints (Cervo et al., 2007; Fuchs-Lacelle, et al., 2004; Hadjistavropoulos et al., 2012). Zwakhalen et al. (2007) show there is a little increase in pain threshold as age advances. However, Mahoney et al. (2008) reported there is no change, or even a decrease, in pain thresholds in older adults. Furthermore, Horgas et al. (2007) reported that age-related loss in the structure and function of the peripheral nerve, and central nervous system (CNS) involved in processing the physical aspect of pain experience, consequently reduces pain sensitivity. 
Mahoney et al. (2008) suggest that age-related changes in pain processing and interpretation may impact the pain sensation in older adults, thereby reducing the ability to self-report pain and putting them at high risk for underassessment and non-treatment of pain. However, the change in pain processing during later life is more complex (Chen et al., 2010), as Horgas et al. (2007) showed that older adults become less able to endure strong pain sensations. Age-related physical/sensory changes in the CNS also explain reduced pain tolerance in the older adults (Hadjistavropoulos et al., 2012). In combination, these findings show that older adults may be more vulnerable to the negative impacts of strong pain.

Despite multiple studies of pain in PWD, it is still clearly unknown if they have excessive changes in physical pain. Some researchers thought that the physical pain may be compromised in PWD because of the disease process of dementia and its related neuropathological changes (Wilson et al., 2008). Also according to Wilson et al., dementia may exacerbate age-related impairments in the physical aspect of pain experience due to the additional burden of cognitive impairment and associated neurodegenerative loss.

However, some studies show that the somatosensory cortex is quite preserved in PWD, and hence the physical component of pain is more likely uncompromised in dementia (Victor, Jensen, Gammaitoni, Gould, White, \& Galer, 2008). Two early studies of pain thresholds in PWD confirmed this preservation of physical pain (Kunz, Lautenbacher, LeBlanc, \& Rainville, 2012). The findings of these studies revealed that there is no significant difference in the pain threshold between PWD and those older adults who do not have dementia.

\section{Affective Pain}

While most physical pain messages are transmitted through the spinal cord to the midbrain, further study has shown that subcentral mechanisms in the thalamus are also activated 
and then advance to the reticular activating system, an area that processes input from the cortex, cranial nerves, limbic system, hypothalamus, and locus ceruleus. This finding lends credence to the idea of an affective aspect of pain experience, because the locus ceruleus, hypothalamus and limbic system are associated with anxiety, fear, stress, and primitive emotion (Wilson, Uhelski, \& Fuchs, 2008).

The limbic system mainly controls the processing of the affective aspect of pain experience. Further pain-modulating processes occur in the midbrain, hypothalamus, and periacqueductal gray. Memory obviously plays a significant role in the processing of pain, affecting anxiety, expectation, and fear (Rudich, Lerman, Gurevich, \& Shahar, 2010).

As shown previously, it is not yet clear if PWD experience significant changes in physical pain; however, affective components of pain experience are often significantly affected in PWD. According to Kovach (2013), PWD experience less affective pain than cognitively intact older adults. Also, they have lower levels of anticipation and arousal for pain. For example, PWD do not experience an increase in heart rate as they are anticipating the pain of venipuncture, but experience increased heart rate after venipuncture (Kovach, 2013).

This diminished processing of affective pain may be explained by recent neuropathologic studies of PWD that have shown some degrees of atrophy in the amygdala, along with atrophy in the frontal cortex and neurofibrillary pathology. These regions of the brain comprise the major components of the limbic system and are mainly responsible for the processing of the affective aspect of pain experience (Kovach, 2013).

Other neuropathologic studies have shown that dementia causes amyloid plaques to form in the amygdala, hypothalamic tracts/nuclei, and thalamic tracts/nuclei. These parts also play a role in the processing of affective pain. Amyloid plaques deform these brain tissues, causing 
impairment in the affective component of pain experience. Furthermore, the psychotic symptoms of dementia may cause further impairments in the structures associated with affective pain (Wilson et al., 2008).

In addition to the neuropathological effects and psychotic symptoms of dementia, the affective pain may be changed by the overall memory loss caused by dementia. According to Rudich et al. (2010), memory loss would surely change the affective pain in PWD. However, the change in affective pain in PWD caused by memory loss is still not clearly understood. Some studies suggest that memory loss could decrease the affective pain in PWD by reducing the anticipation of pain due to the lack of adaptation to painful stimuli (Rudich).

\section{Pain Assessment}

\section{Self-Report Tools}

Although PWD have difficulty reporting their pain, they are still able to use some words to express pain intensity (Horgas et al., 2007; Kovach, 2013; Kovach, Noonan, Schlidt, Reynolds, \& Wells, 2006). They may also use self-report tools such as the FACES scale or a Visual Analog Scale (VAS) (Kovach; Kovach et al.). Many self-report tools are simple or unidimensional. They mainly measure pain intensity, location, and frequency. In self-report tools, pain intensity is reported using a scale from 0 to 9 . The score of ' 0 ' indicates no pain and the score of ' 10 ' indicates severe pain. Other studies use a traditional Visual Analogue Scale (VAS) to report pain intensity by patients themselves (Zwakhalen et al., 2006; Zwakhalen et al., 2012). According to the same studies, the VAS represents a continuum of the subjective feeling of pain to be rated with two ends of extremes. For example, the visual analogue scale would be marked with 'no pain' (0) at one end and 'severe pain' (10) at the other.

Many studies suggest that self-report tools are still reasonably accurate to assess pain in 
the older adults with mild dementia. These self-report tools include the VAS, Numerical Rating Scales, and Faces Pain Scale. The VAS is one of the most frequently used assessment tools in the older adults. Studies have demonstrated that older adults often prefer the VAS when given a choice (Cervo et al., 2007; Fuchs-Lacelle, et al., 2004; Hadjistavropoulos et al., 2012; Horgas et al., 2007; Lin et al.; Zwakhalen et al., 2007). For example, up to $90 \%$ of older adults with moderate dementia were recently shown to be able to use the VAS accurately. Further, even in severe dementia, there were still older adults capable of using this instrument (Lin et al.).

Self-report tools are generally considered reliable and valid among older adults who are able to self-report pain (Husebo et al., 2009; Kovach; 2013, Lin et al., 2011; Zwakhalen et al., 2012). However, it is important to understand that these tools capture the affective experience of pain. In people with dementia who have a decrease in the affective component of pain, these tools may underestimate pain. Studies support that as the severity of dementia increases the use of self-report tools becomes less valid and reliable, and behavioral observations become a more reasonable method to assess pain (Kovach, 2013).

\section{Observational Pain Tools in Dementia}

When older adults with advanced dementia become unable to report their pain, it is recommended that nurses use observation of pain behaviors to assess pain (Kovach et al., 2006). Observational measures are more likely to capture well-established human expressions of physical pain, rather than relying on affective pain experiences or cognitive interpretation of the sensory experience. Therefore, the nurse making the clinical judgments about pain based on these changes needs to know more about the observational pain tools in dementia. Observational pain tools capture changes in behaviors to assess pain in PWD. Assessment of behavioral changes is a process used by a nurse to detect when a person with dementia has may have an 
unmet need such as pain (Kovach et al., 2006). These behaviors include scratching, pushing (Fuchs-Lacelle \& Hadjistavropoulos, 2004), pain words, pain noises, pain faces, rubbing, bracing, and restlessness (Horgas et al., 2012). Findings by Horgas et al. revealed that facial expression is the most common behavior indicator for pain frequently assessed in PWD.

In general, changes in facial expression may help in pain assessment by showing that pain is being experienced (Husebo et al., 2009). The facial changes that arise during pain have been studied for many years (Van Iersel, 2006). Although some variation has been reported, there is substantial agreement across studies that facial changes are likely to occur more frequently and intensively when people experience pain (Cervo et al., 2007; Fuchs-Lacelle, et al., 2004; Hadjistavropoulos et al., 2012; Horgas et al., 2007; Lin et al., 2011; Zwakhalen et al., 2012). Studies of pain and aging have demonstrated that the pain expression remains consistent, even among older adults with mild dementia. Moreover, older adults with advanced dementia have an enhanced pain expression because of diminished inhibitory controls (Mahoney et al., 2008).

The main observational scales used by nurses to assess pain in older adults with dementia are: The Doloplus-2 scale (Chen et al., 2010; Hadjistavropoulos et al., 2012); Certified Nursing Assistant Pain Assessment Tool (CAPT) (Cervo et al., 2007); Pain Assessment in Advanced Dementia (PAINAD) (Lin et al., 2011; van Iersel, 2006); Pain Assessment Checklist for Seniors with Limited Ability to Communicate (PACSLAC) (Fuchs-Lacelle \& Hadjistavropoulos, 2004); Pain Assessment Checklist for Seniors with Limited Ability to Communicate-Dutch PACSLAC-D (Zwakhalen et al., 2012; Zwakhalen et al., 2007); Mobilization Observation Behavior Intensity Dementia (MOBID) Pain Scale (Husebo et al., 2009), Non-Communicative 
Patient's Pain Assessment Instrument (NOPPAIN) (Horgas et al., 2007); and Mahoney Pain Scale (MPS) (Mahoney \& Peters, 2008).

Despite the relatively large number of existing observational tools for the assessment of pain in PWD, some tools have been rated consistently more effective in literature reviews involving psychometric comparisons. Those tools with consistently positive evaluations include the Abbey (Van Iersel, 2006); the Doloplus-2 (Chen et al., 2010 \& Hadjistavropoulos et al., 2012); the PAINAD (Lin et al., 2011;Van Iersel, 2006); and PACSLAC (Fuchs-Lacelle, et al., 2004; Zwakhalen et al., 2007; Zwakhalen et al., 2012). Also, the National Nursing Home Pain Collaborative (NNHPC) has considered the NOPPAIN (Horgas et al., 2007) to have strong psychometric properties. However, there is still no one tool that meets all purposes, and clinicians must consider the evidence and clinical usefulness of recommended tools for their specific population and setting. Many studies have examined psychometric properties across scales and investigated unique characteristics of tools that may make them stronger or weaker from a clinical perspective.

\section{Psychometric Properties}

Reliability. All previously mentioned observational pain tools were tested in nursing homes, and the majority of them had either strong or good internal consistency with an average range $(\alpha=0.75-0.92)$ (Chen et al., 2010; Fuchs-Lacelle \& Hadjistavropoulos, 2004;

Hadjistavropoulos et al., 2012; Horgas et al., 2007; Mahoney \& Peters, 2008; Zwakhalen et al., 2007; Zwakhalen et al., 2012).

Chen at al. (2010) and Lin et al. (2011) reported moderate to very high test-retest reliability for the Doloplus-2 and the PAINAD, respectively. The inter-rater reliability of the MOBID, NOPPAIN, PACSLAC, MPS, and PACSLAC-D scales are considered moderate to 
very high with an average range $(\kappa=0.53-0.94)$, indicating that two different nurses rated the behaviors similarly. According to Chen at al. and Lin et al., inter-rater reliability of the Doloplus2 scale and PAINAD is high $(\mathrm{ICC}=0.86)$. However, the inter-rater reliability of two tools, the PAINAD and PACLAC, has been tested in nursing homes in both pleasant and unpleasant situations. It is important for the assessment tools to be administered at different times of day and during different situations because pain is not constant; it varies throughout the day and according to level of activity (Horgas \& Miller, 2008).

Validity. The validity was not established for all observational pain tools (Cervo et al., 2007; Chen et al., 2010; Hadjistavropoulos et al., 2012; Husebo et al., 2009; Lin et al., 2011; van Iersel, 2006; Zwakhalen et al., 2007; Zwakhalen et al., 2012). Since some observational tools were developed without direct input from PWD and they were used alone, it is difficult to establish their validity. However, some researchers have conducted validity tests for their observational tools, such as the PACSLAC and NOPPAIN (Fuchs-Lacelle \& Hadjistavropouls, 2004; Horgas et al, 2007). According to Fuchs-Lacelle and Hadjistavropoulos, the validity of the PACSLAC was assessed by calculating the correlation between the level of pain intensity measured by the VAS and the total PACSLAC scores $(\mathrm{r}=0.54, \mathrm{p}=.001)$. Horgas et al. assessed the validity of the NOPAIN by calculating its correlation with a Numeric Rating Scale (NRS) (r= $0.66, \mathrm{p} \leq 0.05)$ and a Verbal Descriptor Scale (VDS) $(\mathrm{r}=0.66, \mathrm{p} \leq 0.05)$.

No study has tested the sensitivity and specificity of the NOPPAIN, PACSLAC, and Abbey on nursing home residents. Jordan, Regnard, O’Brien, and Hughes (2012) state clearly that although the PAINAD tool has a high sensitivity (92\%), it has a low specificity (62\%) for pain. The low specificity is due to the fact that the PAINAD tool also measures distress not caused by pain. Although Chen et al. (2010) address the sensitivity and specificity of the 
Doloplus-2 scale, they do not report numerical values of both tests. According to Chen et al, the Doloplus-2 scale had high sensitivity and low specificity in pain assessment.

\section{Remaining Controversy}

Pain Assessment in PWD is still controversial. It is not clear if self-report tools are sufficient to assess pain in PWD if the patient can still self-report. Nurses often use either a selfreported scale or an observational scale to assess pain in PWD. However, some studies show that nurses use a combination of both scales to assess pain and compare the observational scale scores with self-report scale scores to validate them (Kovach, 2013). For those PWD with severe cognitive impairment or communication problem such as aphasia, observational scales are the only reasonable way to assess pain (Cervo et al., 2007; Mahoney \& Peters, 2008). However, no study identifies specific behaviors that may almost certainly capture pain in PWD. Likewise, no study examines the relationship between scores on observational pain tools and self-reported pain.

Self-report is considered the gold standard of pain assessment. Self-report tools are the most reliable measure of pain as long as the person is able to report pain. For PWD, it is recommended that self-report of pain should be attempted regardless of their cognitive impairment (McAuliffe, Nay, O’Donnell, \& Fetherstonhaugh, 2009; Kovach, 2013). Nurses should be responsible for fostering productive discussions about pain with PWD. For example, he or she should be trained in how to determine the patient's preferred pain terminology. PWD, regardless of their ability to self-report pain, could be frequently asked about some pain related term such as discomfort, aching, or soreness. Hence, PWD no longer deny their pain after nurses reword the question with other pain related terms. For example, nurses could ask, "Do you hurt anywhere?" or "Are you uncomfortable?" to rule out pain or discomfort. Once a nurse 
establishes the preferred pain terminology for the PWD they need to document it, communicate it to other healthcare providers, and use it throughout the treatment trajectory (Herr \& Garand, 2001).

It is highly recommended to start self-report pain tools even if a patient has cognitive or communication deficits. Although the ability of PWD to self-report pain may be less reliable over time, recent studies suggest that many older adults with moderate to severe dementia are able to self-report pain reliably when provoked. Another factor that could help foster pain selfreporting is to allow sufficient time for PWD to process the question asked and then formulate a response (Herr \& Garand, 2001). Findings by Snow et al. (2004) indicated that $83 \%$ of PWD in a nursing home were able to respond to a self-report tool, regardless of their ability to self-report pain. According to the study, self-report tools alone are more sufficient to assess pain in communicating PWD. In this particular case, using additional types of pain assessment may cause confusion and inconsistencies among nurses. Also, self-report pain tools are easier to use by nurses, more understandable by patients, and practical and known across the clinical settings (Gregory \& Richardson, 2014).

Still, nursing and medical scientists recognize that self-report alone is insufficient, and observational pain assessment tools are needed for PWD even if they are communicating. Impaired memory and language disabilities caused by dementia impede the ability of PWD to meaningfully self-report their painful experiences. Other confounding factors include delirium, altered level of consciousness, presence of an endotracheal tube, sedatives, and neuromuscular blocking agents (Herr, Coyne, McCaffery, Manworren, \& Merkel, 2011). Furthermore the pathologic changes in dementia seriously affect the ability of those with advanced stages of disease to communicate pain effectively and meaningfully. Damage to the central nervous 
system affects memory, language, and higher-order cognitive processing necessary to communicate the experience (Herr et al., 2011).

When it comes to assessing pain in nonverbal patients, the American Society for Pain Management (ASPM) indicates there is no single pain assessment method sufficient by itself. Therefore, both self-report and observation pain tools are recommended by the ASPM for PWD, regardless of their ability to self-report. Also, according to Huffman and Kunik (2000), pain management is a multidimensional clinical process requiring thorough and systematic assessment. Finally, according to (Gilmore-Bykovskyi \& Bowers, 2013) the use of observational tools for PWD who are able to self-report pain could lead to delayed treatment of pain. In this case, nurses should pursue further, multidimensional assessments and take additional clinical judgment steps.

\section{Conclusion}

Pain assessment in PWD is a critical problem. Since PWD are not able to self-report their pain, they are at great risk for risk for poor treatment of pain. This issue is very serious because it has the potential for multiple negative consequences on health, functioning, and quality of life. For nurses, pain assessment is the first step to manage pain; once they properly perceive and interpret their patients' pain, they are able to commence with the required actions. There are many assessment tools developed which have potential use in the assessment and management process of pain in people with dementia. However, It is not clear if either selfreport tools or observational tools are sufficient alone to assess pain in PWD if the patient can still self-report. Nurses often use either a self-reported scale or an observational scale to assess pain in PWD. 


\section{Manuscript 2: Does Uncertainty Explain Poor Pain Management in PWD? Introduction}

Pain is one of the most prevalent problems in the elderly; pain affects approximately $20 \%$ of the elderly population (Fine, 2009; Gibson \& Lussier, 2012). Chronic pain in patients aged 85 years or older is a common problem, occurring in $40-79 \%$ of individuals (Chen \& Lin, 2008). Fifty-six percent of community-dwelling older adults and $70 \%$ of nursing home residents have pain (Kruger \& Stone, 2008; Takai, Yamamoto-Mitani, Okamoto, Koyama, \& Honda, 2010). This high prevalence of pain among elderly patients is explained in part by physiological agerelated changes and comorbid problems that happen in later life (Fine; Gibson \& Lussier). According to Achterberg et al. (2013), elderly people are more prone to receive poor pain management.

Despite the high prevalence of pain among elderly people, people with dementia (PWD) appear to be at great risk for poor treatment of pain because they have difficulty communicating their unmet comfort needs (Bachino, Snow, Kunrk, Cody, \& Wristers, 2001; Horgas \& Elliot, 2004). According to Husebo et al. (2011), pain affects $50 \%$ to $80 \%$ of Nursing Home (NH) residents with dementia. Several studies have also consistently shown the high prevalence of poor assessment and treatment of pain in NH residents with dementia (Reynolds, Hanson, DeVellis, Henderson, \& Steinhauser, 2008; Williams, Zimmerman, Sloane, \& Reed, 2005; Wu, Miller, Lapane, Roy, \& Mor, 2005). For example, it has been reported that PWD are prescribed significantly less scheduled analgesics as well as received less analgesics than cognitively intact older adults even with controlling for number of painful situations (Achterberg et al., 2013; Kaasalainen, Coker, Dolovich, Papaioannou, Hadjistavropoulos, Emili, \& Ploeg, 2007).

Treatment of pain in PWD goes awry because of: (a) poor assessment, (b) poor treatment, 
or (c) factors relating to nurses' critical thinking and decision-making skills. The purpose of this paper is to provide a brief overview of evidence related to poor assessment and poor treatment, and then to describe in more detail nurses' critical thinking and decision making, with a description and explanation of how the Response to Certainty of Pain (RCP) model (GilmoreBykovskyi \& Bowers, 2013) may help elucidate the how nurse uncertainty regarding suspected pain drives assessment and predicts patient outcomes. The RCP model may help researchers to understand factors that predict poor pain management and may aid in the development of interventions to improve treatment.

\section{Assessment}

There cannot be effective treatment without accurate assessment of pain (Kovach, Cashin, \& Sauer, 2006). Nurse scientists need to give much more attention to developing assessment tools that could improve practice and eliminate barriers to optimal pain management (Kovach, Griffie, Muchka, Noonan, \& Weissman, 2000). A study done by Kovach et al. (2006) identified the need to develop more critical ways to properly assess pain in PWD. It is important that healthcare professionals conduct more appropriate and accurate pain assessment in order to accurately identify pain and provide effective treatment using a range of comforting interventions (Kovach et al., 2008). With a better understanding of the problem of pain, healthcare providers may be able to improve the quality of life for PWD. Over the past few years scientists have made significant progress in this area by developing, evaluating, and introducing a variety of observational assessment tools used to detect pain in PWD (Herr, Bjoro, \& Decker, 2006; Herr, Bursch, \& Black, 2008; Herr, Bursch, Ersek, Miller, \& Swafford, 2010). However, pain is still unrelieved in PWD (Hadjistavropoulos et al., 2011).

Because of the complexity of pain assessment in PWD and the time needed to assess pain 
in this population, nurses may spend a few hours rather than minutes to assess their patients' pain (Prkachin, Solomon, \& Ross, 2007). Nurses may be reluctant to perform a thorough assessment. Instead, these nurses may overly simplify the assessment process by relying on the rapid data processing evident when using intuition to discern pain sufferers from others (Prkachin et al., 2007).

\section{Treatment}

Unrelieved pain in PWD could also be explained in part by poor treatment (McLachlan et al., 2011). Nurses are often concerned about drug addiction and other consequences of analgesic use, such as respiratory depression (McLachlan et al.). Also, nurses may have both knowledge deficits and prejudice with regard to analgesics use and pain in PWD (Kovach et al., 2000). Nurses often struggle with understanding the nature of pain in PWD (Krumm, Larkin, Connolly, Rode, \& Elsner, 2014). They cannot differentiate between the pure sensation of pain and other unpleasant emotions (Krumm et al., 2014). Even if nurses identify suspected pain in a PWD, determining pain location, intensity, and duration is far from a simple exercise (Kaasalainen et al., 2007). According to a recent study, 30\% of PWD residing in nursing homes report pain daily, but $25 \%$ of those nursing home residents with daily pain do not receive analgesics (Adunsky et al., 2002). Even if analgesics are prescribed and available for PWD, nurses often fail to administer those medications, or tend to administer only a portion of the prescribed amount (Adunsky et al.). Moreover, a recent study showed that nurses' analgesic choices are mainly determined by the ability of patients to self-report their pain. As a result, non-verbal patients like PWD have little effect on the choice of analgesics (Gustavsson, \& Sandman, 2015).

Pharmacological treatment is considered to be the first option for pain management (McLachlan et al., 2011). However, nurses may start with non-pharmacological treatment to 
address pain in PWD and delay analgesics. The reason for that is nurses may have misconceptions about behavioral symptoms of pain in PWD. Nurses may conceptualize these behavioral symptoms from a psychological perspective rather than physical perspective. As a result, nurses may not prioritize treatment of physical problems and try instead to treat the nonphysical underlying cause of behavioral changes, such as psychosocial causes (Kaasalainen et 1., 2007; Krumm et al., 2014).

Also, even though nurses use the pharmacological treatment to manage pain in PWD, they may use antipsychotic drugs instead of analgesics. The use of antipsychotic drugs is often associated with decreased level of functioning, unmet care needs, increased serious cardiovascular events, and decreased quality of life (Salzman et al., 2008). Furthermore, some nurses are unclear whether PWD truly have pain or just want to be medicated. In spite of nurses' efforts to manage pain in PWD, they may feel helpless with this group of older adults who have difficulty verbalizing their pain (Liu, Briggs, \& Closs, 2010).

\section{Critical Thinking and Decision Making}

The underestimation of pain assessments and the failure of nurses and others to appropriately treat pain are well known. Far less is known about how nurses think about and make their decision about pain assessment and management in PWD. The problem of pain in PWD may be explained partly by poor critical thinking and decision-making skills on the part of nurses. To make an accurate decision about pain management in PWD, nurses should clearly identify the behavioral indicators of pain, such as facial grimacing, withdrawal, restless behaviors, and negative vocalizations. However, nurses do not have a clear understanding of what the behavior indicators in PWD should look like even in those who could self-report their pain. Instead, nurses often think of autonomic nervous system changes in vital signs, evident in 
acute pain conditions, and observable physical changes as cues to pain in PWD who are having acute or chronic pain (Herr, Coyne, McCaffery, Manworren, \& Merkel, 2011; Herr \& Garand, 2001).

Nursing homes in the U.S. have a high ratio of unlicensed-to-licensed nursing staff (Clark, Jones, \& Pennington, 2004). According to the same study, $80 \%$ to $90 \%$ of care in nursing homes is delivered by Certified Nursing Assistants (CNAs). CNAs often do not receive high quality education and clinical training. Furthermore, the problem of a lack of staffing in nursing homes overwhelms many CNAs and CNAs are not responsible for assessment or clinical decision-making (Buffum, \& Haberfelde, 2007). Consistently, a recent study by Kovach et al. showed that nursing homes are heavily staffed with Licensed Practical Nurses (LPNs) and associate-degree RNs, who, when confronted with the complexity of caring for PWD, may lack adequate training to make decisions regarding appropriate care (Kovach et al., 2012).

According to Kaasalainen et al. (2007), nurses acknowledged that a major problem of pain management in PWD lies in the decision-making process they undergo until they get analgesics prescribed. Nurses believe that the way they interact with pain in PWD is mainly based on a trial and error approach. Nurses often became confused when they were uncertain regarding suspected pain in PWD.

To overcome uncertainties of pain assessment and ambiguities of the pain experience, it has been recommended that nurses identify a variety of behavioral cues to formulate their decisions about pain assessment. These cues are based on the nurses' understanding of the usual behaviors of PWD (Kaasalainen et al.). Unfortunately current pain assessment strategies rarely incorporate formalized decision-making processes. Nurses often look at pain symptoms in PWD as just a manifestation of a behavior change associated with the dementing illness. Regardless of 
how different PWD show pain, it is discussed as and responded to as a sign of a dementing illness. The current body of evidence suggests that nurses often do not have a clear understanding of what pain looks like in PWD, which then leads to a state of uncertainty. This uncertainty impacts the nurses' decision-making in regards to pain treatment (Kaasalainen et al.). This paper will present theoretical and empirical evidence supporting the premise that nurses' uncertainty regarding suspected pain in PWD may explain inadequate assessment, which then leads to under-treatment of pain.

\section{Background to Decision Making Theory}

\section{Background of Field of Healthcare Decision-Making}

Decision-making about treatment is an essential part of modern healthcare (Lally \& Tullo, 2012). Dementia causes a gradual decline in decision-making ability. Therefore, PWD may be unable to make decisions related to their treatment (Smebye, Kirkevold, \& Engedal, 2012). Healthcare providers play a significant role in making decisions about different treatment options, based on information regarding risks and benefits (Lally \& Tullo). In the case of dementia, the role of healthcare providers is even more significant. Many researchers have described how nurses acquire new knowledge, apply acquired knowledge to clinical practice, integrate past learning with new learning, acquire knowledge derived from clinical practice, make decisions and use decision-making processes to transfer theoretical knowledge into practice (Smebye et al., 2012). In the following paragraphs, this paper will provide an overview of some of the main decision-making models used in the field of healthcare.

\section{Overview of Core Nurse Decision-Making Models}

The implementation of evidence-based interventions in healthcare settings has increased the importance of understanding the nursing decision-making process. A review of the literature 
revealed that many decision-making theories have been applied in healthcare settings. In the nursing field, several theoretical frameworks have been used to study the nurses' decisionmaking process, since there is a noticeable knowledge deficit among nurses about how to make decisions to improve quality of healthcare.

Cognitive continuum theory (CCT). The Cognitive Continuum Theory (CCT) has been used to facilitate nursing decision-making processes in multiple clinical settings (Cader, Campbell, \& Watson, 2005). The CCT is a descriptive theory that explains the relationship between a decision-making task and an individual's cognition. The CCT consists of several concepts, including modes of cognition, task properties, modes of inquiry, pattern recognition, functional relations, oscillation, and alternation. The concept of modes of cognition is associated with three main components, including analysis, intuition and quasi-rationality. Analysis has been defined as slow data processing, high conscious awareness, and a consistent process that includes a task-specific organizing principle and high confidence in the method. Intuition is defined as a rapid data processing, low conscious awareness, and low consist process that includes an average task-specific organizing principle and low confidence in method. Quasirationality is considered to be the central area in the cognitive continuum that moderates the interaction between the other two modes of cognition: intuition and analysis (Cader et al., 2005).

The concept of task properties has three components, including complexity, ambiguity, and presentation. Complexity concerns a number of information cues, redundancy of cues, and the principle for combining information. Ambiguity concerns the existence of a principle to organize information, familiarity with content, and potential for accuracy in judgment. Presentation concerns the potential for decomposition into sub-tasks, visual or quantitative presentation, and time available to undertake the task. There are two dimensions for task 
properties: well-structured and ill-structured task. Well-structured tasks induce analysis through decomposing the tasks into many sections. Although this dimension takes time to resolve the task, it has a high degree of certainty. On the other hand, ill-structured tasks induce intuition through decomposing the task into a few sections. Although this dimension does not take time to resolve the task, it has a low level of certainty. Finally, the CCT consists of six modes of inquiry. The first three are related to analysis and the last three relate to intuition (Cader et al., 2005).

While the CCT theory does a good job of differentiating between analytical and intuitive processes needed to make decisions, the processes described are quite linear and fail to accomodate the multi-factorial nature of pain in PWD. Although analytical process is justifiable, it is complicated. On the other hand, although intuitive process is quick and flexible, it is imprecise and irretraceable. Intuitive errors happen unexpectedly and are often hard to identify (Dhami \& Thomson, 2012). Also, The mode of cognition that CCT use lacks rationality and overly relies on intuition, which has been viewed as the basis for irrational acts or guessing. The cognitive complexity of nurse prescribers' decision-making suggests that intuition is insufficient for nurse prescribing. Futhermore, the model does not account for or capture uncertain or ambiguous thinking in the decision making process (Offredy, Kendall, \& Goodman, 2008). Research using the CCT has involved small samples, with weak designs and no research has examined the association between the nurses' decision making certainty and assessment type with agitation and pain levels in PWD.

Adaptive pain management (APM). Even though more treatment options and new medications have been developed, it is still unclear how healthcare providers decide what is the most clinically effective treatment plan for elderly patients. Such decision-making processes can 
be subjective and depend on patients' information and health care providers' experiences. In pain management, patients will experience different pain outcomes depending on the time of diagnosis and initiation of treatment. Adaptive Pain Management (APM) is a decision-making framework that aims to frequently review patients' past and current information to identify the best choice of treatment (Lin, LeBoulluec, Zeng, Chen, \& Gatchel, 2014).

The APM demonstrates a proof-of-concept and develops the basic process for creating an adaptive treatment of pain by demonstrating a decision support system based on dynamic programming. The APM has two main stages that end with DP formulation and solution process presentation. The first stage, prior to treatment, includes an evaluation of the patient's pain characteristics, related health parameters and medical history, and ends with the determination of a treatment regime. The second stage determines whether an adjustment in the treatment should be made or not, and comes up with the final outcomes. The decision in each stage is the available treatment plans, which could be a combination of a number of different pharmacological or non-pharmacological interventions (Lin et al., 2014).

While the APM theory does a good job of identifing the best choice of pain treatment in eldelry patinets and demonstrating a decision support system based on dynamic programming process, the processe implies that nurse should continually adapt and readapt the treatment to the patient (Lin et al., 2014). This continouse adaptation and readaptation of treatment relies on patient feedback which means that patents should be able to verbalize their unmet needs. Therefore, the model does not account for cognitive impairemnt and communication deficit in PWD (Dawson \& Lavori, 2008). Also, the model does not account for or capture uncertain or ambiguous thinking in the decision making process. Research using the APM has involved small samples, with weak designs and no research has examined the association between the 
nurses' decision making certainty and assessment type with agitation and pain levels in PWD (Dawson \& Lavori, 2008).

\section{Uncertainty and Pain Assessment}

Two theories have directly addressed the role of uncertainty in pain management in PWD. A model by Shields et al. states that the decision-making process is a complex intellectual process of clinical reasoning or judgment that includes formulating interpretations regarding what level of certainty nurses have in taking action. Action refers to the interventions the nurse chooses or does not choose to do. To take no action is a conscious decision not to initiate an intervention. Both actions and non-actions are the result of the decision-making process, and both involve a process of interpretation (Shields et al., 2013). While the work of Shields et al. posits that when recall of nursing education and training, theoretical and experiential knowledge, personal experiences, culture, values, and beliefs to the situation may increase or decrease the level a nurse's level of certainty about pain in PWD, explanations within the model are not well developed, and lack direct applicability to clinical situations of complex assessment of pain in PWD.

\section{Introduction to the Response to Certainty of Pain Model (RCP)}

The Response to Certainty of Pain model (RCP) by Gilmore-Bykovskyi and Bowers (2013) (figure 1) illustrates two trajectories for nurses' assessment and treatment of pain for nursing home residents: one when the nurse is certain the patient is in pain and one when the nurse is uncertain whether the patient is in pain. When the nurse is certain the patient is in pain the trajectory moves in a timely fashion from suspected pain to treatment. When the nurse is uncertain the patient is in pain the trajectory from suspected pain to treatment is delayed or never accomplished (Gilmore-Bykovskyi \& Bowers). 
The uncertainty trajectory is often slowed down by a time consuming assessment process based on trial and error. Certainty of pain is quickly confirmed by prompt treatment in the certainty trajectory. In the uncertainty trajectory, although various treatments may be tried, the nurse may never be certain that the patient was in pain because the path from suspected pain to treatment is more convoluted. Furthermore, in many cases the path never leads to treatment so there is no treatment outcome to confirm the presence of pain (Gilmore-Bykovskyi \& Bowers, 2013).

Figure 2: The Response to Certainty of Pain model (RCP)

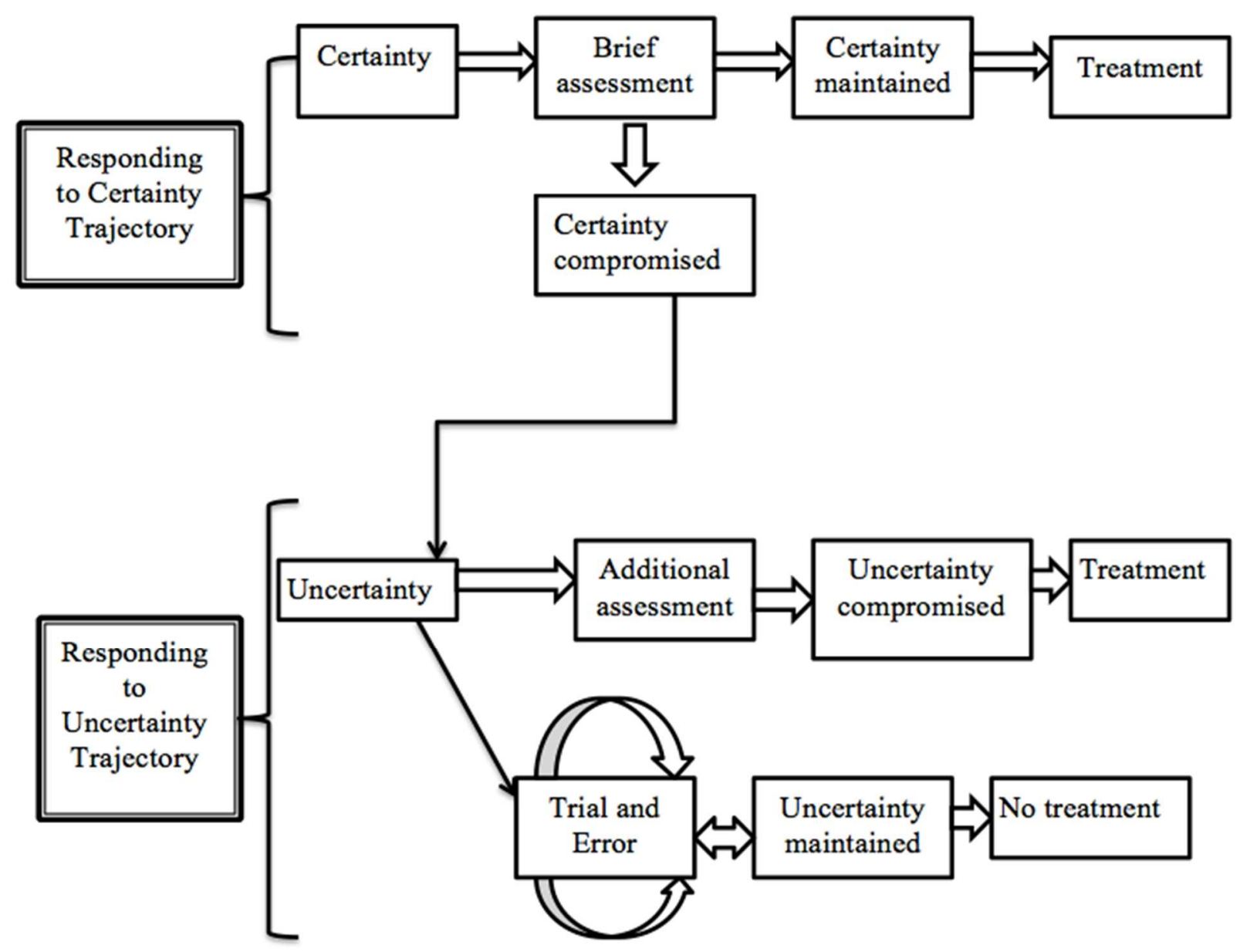


Gilmore-Bykovskyi and Bowers' model of how certainty and uncertainty create different trajectories for nurses' decision-making in pain management for nursing home patients could lay the foundation for much needed translational research to improve nursing practice for patients with dementia (Gilmore-Bykovskyi \& Bowers, 2013). The RCP model demonstrates that the role of certainty should be a key nursing factor in future gerontological pain research.

\section{Nurses' Certainty Trajectory}

When nurses are certain regarding suspected pain they do a brief self-report assessment and provide timely pain management. According to the RCP model, nurses implement timely pain management if residents do not have dementia because their behaviors are highly suggestive of pain. Also, nurses respond with a high level of certainty regarding suspected pain in patients with a short-term stay in the nursing home, even though nurses know that not all short-term stay residents are cognitively intact.

According to Gilmore-Bykovskyi and Bowers (2013), nurses try to confirm suspected pain in older adult patients who do not have dementia by asking the patient for a self-report. In case of PWD, nurses become more certain regarding suspected pain and provide timely treatment if PWD show obvious pain indicators. When nurses feel a high level of certainty about pain in their PWD they are more likely to quickly validate their assessments and to confirm their certainty about the presence of pain.

\section{Nurses' Uncertainty Trajectory}

Nurses are uncertain regarding suspected pain in PWD for a number of reasons. The complexity of pain assessment in PWD does not enable nurses to suspect pain in their patients. Furthermore, the severity of dementia strongly affects the level of a nurse's certainty. As the severity of dementia increases, a nurse's level of certainty regarding suspected pain decreases 
(Gilmore-Bykovskyi \& Bowers, 2013). Moreover, nurses may not suspect pain in PWD because of the inconsistency of their behavior and because pain may not be the primary cause of their behavior.

According to the RCP model, when nurses are uncertain regarding suspected pain in PWD, they do not confirm certainty and evaluate the effectiveness of the pain treatment given to PWD. Nurses do not try to ease their uncertainty by asking PWD if they are in pain, because PWD cannot verbalize their pain. Instead, nurses try to ease their uncertainty by conducting additional assessment to increase certainty and doing trial and error process. In this case, treatment is focused on reversing behavioral changes and consequently the treatment of pain may be delayed or not provided at all. Also according to the model, nurses are consistently uncertain regarding suspected pain in elderly with long-term stays. Those elderly usually have many comorbid problems that make nurses unable to suspect pain because they believe pain generally is not anticipated in a long-term stay. In this situations nurses also do further assessments, and include additional decision-making processes. As a result, the treatment of pain may be delayed.

\section{Other Theoretical References}

PWD are unable to provide reliable verbal information about their pain experience. Therefore, they are unable to confirm nurses' certainty or uncertainty regarding suspected pain. As a result nurses might refer to their interpretations of clinical data obtained from observing, listening, and evaluating pain cues. Pain cues are defined as changes in behaviors such as aggression and restlessness/agitation, sounds such as vocalization, or appearance such as facial expressions (Tait et al., 2009). Recognizing these behavioral changes needs additional pain assessment and more decision-making steps. The nurses' ability to interpret these changes is limited, impeding them to make certain decisions about pain treatment (Parke, 1998). 
Nurse also may unable to discriminate between pain and dementia (Kaasalainen et al., 2007). Nurses acknowledge that they are often uncertain about pain in PWD, as they are unsure whether behavioral changes are pain-related or dementia-related. In most cases, nurses believed these behavioral changes are caused by something other than pain. Indeed, the last thing that nurses think about when they care for PWD who show change in behaviors is pain. Consequently, nurses would try to manage these behavioral changes based on a pragmatic approach rather than theoretical considerations. As nurses often do not have a definitive diagnosis of pain in PWD, they struggle with treatment options and frequently question their decisions about chosen treatment of pain.

When nurses are uncertain regarding suspected pain in PWD, they tend to use their intuition. In this case, nurses not only cannot recognize the behavioral changes in PWD but they are also unable to clearly translate their intuition into meaningful data, facilitating clinical decisions about pain (Shega et al., 2006). For example, nurses may report that they just feel inside something is wrong because the patient is not being herself. In this example, nurses' intuition is not enough to develop certainty regarding suspected pain in PWD (Shega et al). When nurses use their intuition to assess pain in PWD, they are uncertain if the behavioral changes they observe represent pain.

Also, nurses do not have clear understanding of decision-making processes and other relevant information. Understanding other relevant information includes diagnoses and medical histories, the nurses' knowledge of pathophysiology, and the nurses' personal experiences with painful conditions. The decision-making process involves recognizing the PWD's specific pain cues, effectively assessing pain, administering analgesic in a timely manner, and evaluating the effectiveness of the intervention. However, nurses use a process of trial and error process 
instead. This process involves administering nonpharmachological intervention and observing PWD's responses to these interventions or just looking for the reversal of behavioral changes (Sheilds et al., 2013).

\section{Evidence That Relates to Uncertainty and Pain Treatment}

Because healthcare providers are uncertain regarding suspected pain in PWD, PWD are prescribed significantly less analgesics (Achterberg et al., 2013). For example, when physicians prescribe analgesics for PWD after hip fracture surgery, they put them at a dosage that is onethird of that prescribed for cognitively intact patients (Achterberg et al.). Furthermore, when physicians are uncertain about pain in PWD, they start with a very low dose of analgesics and then increase the dosage if a low dose does not work. However, physicians and nurses are unclear about whether and how much they should increase the dosage of analgesics. They may start to think that maybe it is not actually pain; maybe this is part of dementia, not pain (Kaasalainen et al., 2007).

Furthermore, even though physicians may prescribe analgesics for PWD, nurses may sometimes be uncertain about pain, and consequently, PWD may not receive adequate pain treatment. A recent study by McCaffery, Ferrell, and Pasero found that nurses' uncertainty regarding suspected pain in PWD influenced the choice of opioid analgesics dose and contributed to poor treatment of pain. Similarly, a study by Kaasalainen et al. (2007) found that nurses were not always certain about their ratings of pain, which were not significantly correlated with the administration of analgesics. Consequently, even though PWD have pain in the past, they do not always receive analgesics (Kaasalainen et al.).

When nurses are uncertain about pain in PWD, they feel uncomfortable and hesitant to use analgesics to treat pain. They tend to administer analgesics only after all other non- 
pharmacological interventions have been tried and proven ineffective. According to a recent study, nurses who were uncertain whether a patient's behavior indicated pain were less likely to start pharmacological treatment than nurses who had higher levels of certainty (Achterberg et a., 2013). Nurses who were uncertain about their patient's pain chose other pain interventions, delaying more effective pharmacological treatment. These other pain interventions include toileting, changing the room to fit to resident preferences, providing acetaminophen, changing position, regulating the temperature of environment, reducing stimulation, doing outdoor activities, and providing food. Nurses and physicians are not willing to take the risks to treat pain with opioid analgesics. This reluctance to use analgesics results from their uncertainty regarding suspected pain in PWD (Kaasalainen et al., 2007).

\section{Future Research and Further Theoretical Development}

Further theoretical development is needed to understand the role of experience on nurses' certainty. It unclear yet whether nurses who have more experience are more certain about pain in PWD or not. According to Kovach et al. (2000), nurses who have more experience in caring for PWD clearly understand behavior changes in PWD, and are more certain about suspected pain. Also, Parke examined nurses' certainty about pain in PWD and found that having relationships and experiences with PWD results in a certain clinical decision about pain. However, According to Gilmore-Bykovskyi and Bowers (2013), nurses are more certain about pain in PWD who have short stay. Therefore, the relationship that nurses could establish with PWD will be not too long. So as nurses have more experience with PWD, they become less certain about the pain that PWD have.

When making a clinical decision on pain assessment or treatment in PWD based on intuition, it is important to be aware of biases that may confound nurses' certainty. Unfortunately 
some recent studies show nurses may often introduce bias into the decision-making process about pain, which then leads to uncertainty. These studies indicate there is a tendency for experienced nurses to rely more heavily on their intuition, and thus have more bias than those with less experience. This bias is enhanced among observers with clinical experience with pain because of overexposure to evidence of pain in others. The effect of experience suggests a researchable question. Future research is needed to elaborate more about the possible causal pathway that connects nurses' experience and their certainty about pain in PWD (Pretz, \& Folse, 2011).

\section{Conclusion}

PWD are at great risk for unrelieved pain due to poor assessment, inadequate management, or problems with decision-making process. Many decision-making theories including the CTT and APM have been developed on pain management recently. However, The $\mathrm{RCP}$ is the only theoretical framework that has examined the association between the nurses' decision making certainty and assessment type with agitation and pain levels in PWD. The RCP provides an understanding of treatment decisions regarding pain in PWD. This article provides further evidence that nurses' uncertainty may explain or predict poor treatment of pain in PWD. 


\section{Chapter Summery}

A detailed literature review of pain assessment in PWD as well as explanation of the impact of uncertainty on pain treatment in PWD are presented in this chapter. An overview of core nurse decision-making models is also provided in this. Also, this chapter provides overview of other theoretical references and empirical evidence on uncertainty and pain assessment and treatment. This chapter provides the familiarity necessary to fully understand the concepts under investigation in this study. 


\section{CHAPTER 3}

\section{Introduction to Chapter}

The purpose of this study is to examine the association between the nurses' decisionmaking certainty, assessment scope and the outcomes of agitation and pain in nursing home residents with dementia who display a change in behavior. The research methods used to gain information about nurses' certainty/uncertainty regarding suspected pain, assessment scope, and the level of pain and agitation in PWD are described in this chapter. The design, sample selection procedure, details of instruments utilized, data collection methods, and data analysis techniques are explicated. The hypotheses and research questions that guide this study can be found on pages 16 to 17 in this chapter.

\section{Method}

\section{Research Design}

A descriptive-correlational design was used to understand the factors that are associated with the assessment and treatment of pain in nursing home residents with dementia in Jordan. The researcher does not want to apply control over or manipulate the variables being studied. The study also does not measure a causal relationship between variables. At this point, the main focus of the study is to describe the problem of pain assessment and management in PWD. A descriptive-correlational design allows the researcher to describe selected variables and study associations between groups. It was chosen for this study to compare the characteristics of groups according to selected variables. The variables of this study were selected on the basis of theory and previous studies where assessment scope and nurses' certainty/uncertainty have been associated with pain and agitation (Chen et al., 2010; Gilmore-Bykovskyi \& Bowers, 2013; Shega et al., 2006; Shields et al., 2013; Tait et al., 2009). This study would add to nursing 
knowledge of PWD by examining how clinical variables that predict pain and agitation in people who have known pain or a painful diagnosis and a change in behavior or condition. Results of this study would help determine whether nurses' uncertainty regarding suspected pain can explain poor management of pain in PWD. This study could inform the development of an intervention, a standard guideline of pain care for PWD, changes in practice or policy related to the assessment and treatment of pain in PWD in Jordan.

\section{Setting}

This study took place at five different nursing homes, located in the capital of Jordan, Amman. The average bed capacity of these nursing homes is 250 beds with $55 \%$ of residents having dementia. All five nursing homes are regulated by the same administrative director who is hired by the social welfare minister of Jordan. According to Jordanian health social welfare ministry, the main goal of all five nursing homes is to ensure highly effective palliative care for a group of Jordanian older adults who suffer from many comorbid problems. These five nursing homes are located in an ethnically and culturally homogenous area in Amman city, Jordan. The city of Amman is chosen by convenience for geographic closeness to the researcher and also because this is the target population of interest. Use of nursing homes provides feasible access to people with dementia and known pain or a known pain diagnosis. Drawing from the same ethnic and cultural population limits generalizability but controls some potential confounding effects.

\section{Sample}

A convenience sample of nursing home residents who have dementia and known pain or a known pain diagnosis was recruited to participate in this study. According to Burns and Grove (2007), subjects participating in a study through the convenience sampling method, 
happen to be in the right place, at the right time; they are available solely by accident. Given time, access and logistical constraints, collecting meaningful information from a random sample from nursing home residents in Jordan would not be feasible. This study uses the method of convenience sampling because it is faster, easier, more readily available, and more cost effective.

Sample size was determined after meeting with a statistician and doing a power analysis. By using power analysis to determine the sample size, the probability to commit Type II error is minimized (Polit \& Beck, 2012). The statistician used A-priori Sample Size Calculator for Multiple Regression (Cohen, Cohen, West, \& Aiken, 2003) to calculate the sample size. Given an alpha level of 0.05 , three predictor variables, an anticipated effect size of 0.15 , and the desired statistical power level of 0.8 , the minimum required sample size is 75 .

The inclusion criteria of the study are: (1) NH residents who are aged 55 and above. Older adulthood in Jordan is most commonly defined at age of 55 years (Al-btoush, 2012; BaniIssa, Almomani, \& Eldeirawi, 2014). (2) NH residents with known pain or known pain diagnosis. Known pain was determined if older adults have any disease, injuries, or problems that usually cause pain for older adult people. These problems include degenerative joint disease, fractures, pressure ulcers, neuropathic pain, urinary retention, post-stroke syndrome, improper positioning, cancer pain, contractures, oral/dental sources, and constipation. (3) $\mathrm{NH}$ residents whose family members or caregivers live in the same geographical area of the study in Jordan, Amman to facilitate the consent form procedures. (4) NH residents with any degree of memory loss or cognitive impairment as documented in medical charts or reported by nurses in case medical charts are incomplete. (5) Nurses with a minimum of one-year experience in nursing homes and working full time (at least 30 hours a week). 


\section{Instruments and Operational Definitions}

\section{Outcome instruments.}

Pain. Pain is defined as "A negative emotional and/or physical state subject to variation in magnitude in response to internal or environmental conditions" (Hurley et al., 1992, p. 374). The sensation is associated with actual or potential tissue damage and is mediated by specific nerve fibers. Pain is operationally defined as behavioral indicators expressed by a negative emotional (Affective) and/or physical status such as noisy breathing, negative vocalization, sad facial expression, and frown. This operational definition is measured by the Discomfort ScaleDementia Alzheimer Type (DS-DAT) scale. The scale uses the same conceptual and operational definitions that this study adopts. The assumption of the tool is that pain can be measured by observed markers (behavioral indicators) of internal state (negative physical and emotional status). The DS-DAT enables the researcher to operationalize pain using the best method available to capture the conceptual definition of pain. Since we are interested in measuring generalized pain, this tool is an appropriate conceptual fit.

The Discomfort-DAT is a nine-item observational measure that uses a 4-point Likert scale. Since PWD often cannot accurately complete self-report tools, we chose to use an observational tool. The Discomfort-DAT was designed for use with this population and yielded ordinal level data. The tool has 7 negative items (e.g. 'tense body language'), and 2 positive items (e.g. 'content facial expression'). The score of each item ranges from 0 to 3 , and the total score ranges from 0 (no discomfort) to 27 (high discomfort level). Scores are based on frequency, intensity, and duration of the observed behavior over 5 min following a 15 minute washout period from stressful events. Its complexity requires training in proper use of the instrument (Hurley, Volicer, Hanrahan, Houde, \& Volicer, 1992). The administration of the tool 
was done two times for each resident.

Several studies have demonstrated acceptable reliability and validity of the tool (Van Der Steen, Pasman, Ribbe, Van Der Wal, Onwuteaka-Philipsen, 2009). Internal consistency, alpha coefficients of the tool ranged between .77 and .82 and the interrater reliability was kappa .85 (Kovach, 2012). To minimize measurement error, the researcher was strictly be consistent in all data collection methods and obtain interrater reliability checks for every $10 \%$ of data collection.

Agitation. Agitation is not considered a diagnostic term, but is used by clinicians to describe a group of behavioral symptoms that may reflect an underlying disorder. According to Cohen-Mansfield (1997), agitated behavior can be manifested in three ways: (1) abuse or aggression toward self or others; (2) appropriate behavior performed with inappropriate frequency, such as constantly asking questions; or (3) inappropriate according to social standards for the specific situation, as in taking off clothes in the activity room (Cohen-Mansfield, Marx, \& Rosenthal, 1989). Cohen-Mansfield has defined 30 agitated behaviors that are measured by the Wisconsin Agitation Inventory (WAI) scale (Kovach et al., 2004). The scale uses the same conceptual and operational definitions that this study adopts. The assumption of the tool is that agitation could be measured by observed verbal and behavioral markers. The scale enables the observer to operationalize the concept using a highly reliable method to capture the range of agitated behaviors defined by Cohen-Mansfield.

Agitation was measured with the Wisconsin Agitation Inventory (WAI), a visual analog scale, with scores ranging from 0 to 100 . This tool assesses agitation through direct observation of agitated behaviors. The observational approach decreases the measurement error associated with retrospective recall bias. Several studies have demonstrated acceptable reliability and validity of the tool. According to Kovach et al. (2012), the interrater reliability achieved for 
WAI was kappa $=.87($ Kovach, 2012). The administration of the tool was done at four times for each resident. This tool requires a 3-minute observation period following a 15-minute washout period from stressful events. To minimize measurement error, the researcher was consistent in all data collection methods and obtain interrater reliability checks for every $10 \%$ of data collection.

\section{Predictor instruments.}

Tracking forms are used by nurses to record date and time of behavior or condition change, describe the change, rate pre and post-assessment level of certainty and document assessments and treatments delivered (Appendix X). To assure valid and complete tracking of information, the tracking forms were crosschecked with the nurse's reports and resident medical records to investigate and resolve discrepancies.

Nurses' certainty. Certainty/uncertainty regarding suspected pain is defined operationally as the amount of certainty or uncertainty that nurses perceive when they assess PWD for suspected pain. There is no standard tool available that specifically measures nurses' certainty of pain. Nurses' certainty was measured with a 1-item scale (score1-5) with the following responses: "very certain", “certain", "don't know”, "uncertain”, and "very uncertain". This type of scaling is an extremely popular means for measuring attitudes, positions, and decisions. Nurses indicate their own attitude, position, decision about suspected pain in PWD along the scale. The scale enables the observer to operationalize the concept using the best method available to capture the conceptual definition of certainty; self-report.

Assessment scope. Assessment scope is defined operationally as a range of types of pain assessment provided to PWD. This operational definition was measured by reviewing the nurses' Tracking form assessment notes to identify the range of tools and types of assessments nurses 
used to determine their patients' pain levels, from no assessment to behavioral and functional assessment. Kovach (Kovach, Logan, Simpson, \& Reynolds, 2010) has used this measurement technique successfully in other studies. Though time-intensive, good inter-rater reliability has been achieved and the measure has been sensitive to change. Inter-rater reliability by two raters during the Kovach 2015 study for assessment quality was .84 for 60 assessments. Assessment scope was coded based on the following coding rubric:

$$
\begin{aligned}
& 0=\text { no assessment } \\
& 1 \text { = brief self-report } \\
& 2 \text { = numerical scale } \\
& 3 \text { = face or color scale } \\
& 4=\text { behavioral assessment (with or without self-report) } \\
& 5 \text { = functional assessment (with or without self-report) } \\
& 6 \text { = combined behavioral and functional assessment (with or without self-report) } \\
& \text { Self-report alone is the defining characteristic of unidimensional tools for pain }
\end{aligned}
$$
assessment. Hence, scores of 1-3 was classified as unidimensional when nominal level comparisons of differences between unidimensional and multidimensional assessment are completed. Multidimensional assessment may or may not utilize self-report but is distinguished by nurses' observation of behavioral changes in their patients (assessed with or without movement) and/or functional changes that are common signs of pain (Gilmore-Bykovskyi \& Bowers, 2013). Hence, scores of 4-6 was classified as multidimensional when treated as a nominal level variable. To minimize measurement error, the researcher was consistent in all data collection methods and obtained inter-rater reliability checks for every $10 \%$ of data collection. 


\section{Other instruments.}

Severity of dementia. Severity of dementia was measured with the Mini Mental Status

Exam. It consists of 30 questions, measuring orientation, registration, recall, attention, calculation, and language (Ellis, 2006). The range of scores is $0-30$. Higher scores indicate greater cognitive ability. This tool offers a quick and simple way to quantify cognitive function and screen for cognitive loss. Several studies have demonstrated acceptable reliability and validity of the tool supporting its use (Ellis).

Comorbid burden. Comorbid burden was measured using the Cumulative Illness Rating Scale for Geriatrics (CIRS-G) (Lin, Lin \&, Gurel, 1968). The scale captures 15 of the more prevalent clinical conditions that are graded on 0-4 disease severity scale to yield ordinal level data (Borson, Scanlan, Lessig, \& DeMers, 2010). The CIRS-G was measured one time following consent. It is designed to provide a good evaluation of all clinical data pertinent to illnesses in all body systems. In addition, it is designed to rate the severity of illnesses. The scale has high inter-rater reliability. It is also considered as 'gold standard' for rating total burden of comorbid illnesses in older adults. The CIRS-G is much more sensitive to the effects of age and cognitive impairment than other comorbidity measures. Also, it is the most reliable and valid comorbidity tool in the presence of dementia (Borson et al., 2010).

Ability to verbally self-report symptoms. The ability of PWD to whether or not still verbally self-report symptoms was measured by Communication of Need-Dementia Alzheimer's Type (CON-AD) tool (Kovach \& Venes, 2003; Personal Communication, 2015). Content validity of the scale has been established and a Cronbach alpha was calculated for this scale. Additional possible variables including gender and age were measured by looking in medical records. 


\section{Human Subjects Considerations}

Approval for the study was obtained from the Institutional, Review Board (IRB) of the University of Wisconsin-Milwaukee (UWM). Jordan does not have an IRB and we have been working with the UWM IRB to develop procedures that met IRB requirements and protect human subjects. To access nursing homes in Jordan, a letter of support is needed from the Social Welfare Ministry of Jordan, showing their interest to host the researcher at their nursing homes and provide him with the space and access to recruit the study participants. In order to obtain IRB approval for this international study, the researcher obtained a letter of support from the minister of Social Welfare in Jordan and a letter of support from Jordan University of Science and Technology, the researcher's sponsoring institution. The minister of Social Welfare in Jordan also issued a letter to the directors of nursing homes to facilitate access to selected nursing homes for this research. The directors of five nursing homes have been contacted by the researcher via phone call to explain the study objectives and research process and they have expressed their willingness to cooperate in the study. At their request, the researcher emailed each director the proposal for the study. After this research proposal was approved, the researcher did seek additional IRB approval from UWM.

Written informed consent was obtained from the participants following UWM IRB approval and prior to completing any study-related information. Both residents and nurses were research participants in this study and provided informed consent. Those residents who legally provide their own consent had the consent form read and explained to them. For those NH residents who were unable to make their own decision, the power of attorney provided written consent and the resident provided verbal assent. All people providing consent or assent had an opportunity to have their questions answered. The consent form emphasized the privacy 
protections afforded participants in this study. The voluntary nature of the study was emphasized. Protected Health Information (PHI) was not disclosed to anyone by the researcher without written consent from the participant.

The participants were assigned an identification number (ID). Confidentiality was maintained by using ID numbers instead of participant's name. No identifying information was written on the data collection sheets. The researcher kept records in a locked and secured location which was accessible only to him. Computer data was coded by ID number and was not contain any identifying information. Data was stored on a password-protected computer. Only the researcher and research assistant had access to code numbers that link information to a participant's name.

\section{Consent and Research Procedures}

Table 1 provides a summary of on-site consent and research procedures. The orientation for this study involved a presentation about the study process and significance to the nursing staff of the nursing homes. The orientation also involved setting up the schedule for the onsite visits. Visits to the study sites were scheduled at the convenience of the nursing home directors and nursing staff. These site visits were at least twice a week ranging from about 6 to 8 hours per visit.

Table 1 Summary of On-Site Consent and Research Procedures

\begin{tabular}{|l|l|}
\hline Timing, Procedures/Measures & By Whom \\
\hline Orientation & -Mohammad \\
- The study process and significance were presented to staff & \& NH \\
- Set up date/time of visits & $\begin{array}{l}\text { administrative } \\
\text { staff }\end{array}$ \\
\hline
\end{tabular}




\begin{tabular}{|c|c|}
\hline Timing, Procedures/Measures & By Whom \\
\hline $\begin{array}{l}\text { Eligibility and demographic description: } \\
\text { - Staff conduct chart review for diagnoses and home addresses } \\
\text { - Year of nurses' experience and workload from interview with NH director. } \\
\text {-List of nurses and residents was created } \\
\text {-Send out and get consents---after consent } \\
\text {-Demographics: length of stay, age, gender from medical record } \\
\text { - Mental status exam from interview. } \\
\text { - CIRS-G----any time is convenient not eligibility; form medical record } \\
\text { - CON-AD ----any time is convenient not eligibility; from nurse report }\end{array}$ & $\begin{array}{l}\text {-Staff } \\
\text {-Mohammad }\end{array}$ \\
\hline $\begin{array}{l}\text { Data Collection } \\
\text { (A)Change Tracking forms } \\
\text { - Training of nurses in completion of Tracking Forms. } \\
\text { - Preassessment level of certainty was recorded by nurses once they notice a } \\
\text { change in resident's behavior or condition. Then, type of assessment provided to } \\
\text { the resident was recorded. After that, post assessment level of certainty was } \\
\text { recorded. } \\
\text { (B) Pain and agitation outcomes: } \\
\text { Observational measures were completed after } 3 \text { and } 7 \text { days of change in } \\
\text { behavior or condition using time collecting for } 2 \text { collections of Discomfort-DAT } \\
\& 4 \text { collections of Wisconsin Agitation Inventory. Both tools were collected in } \\
\text { midday at least } 15 \text { minutes after the time of any potentially discomfort- or } \\
\text { stress-producing event. }\end{array}$ & $\begin{array}{l}\text {-Mohammad } \\
\text { and RA }\end{array}$ \\
\hline $\begin{array}{l}\text { - Two } 3 \text { minutes observations of agitation were done with at least } 10 \text { minutes } \\
\text { apart. } \\
\text { - The } 1^{\text {st }} \text { observation of agitation was measured and while waiting for } 10 \\
\text { minutes to do the second observation of agitation, the } 1^{\text {st }} \text { observation of pain } \\
\text { was measured. } \\
\text { - After doing the } 2^{\text {nd }} \text { observation, the researcher waited for } 30 \text { minutes to do the } \\
2^{\text {nd }} \text { round of pain and agitation observations. }\end{array}$ & \\
\hline
\end{tabular}

Eligibility. The researcher discussed the eligibility criteria with the directors of the

nursing homes to determine eligible clients to be included in the study based on clients' history, medical record, and measurement tools. Consent forms were distributed to the participants, caregivers, family members, or a legally authorized representative of residents with a DPOA.

The researcher enlisted nurses to explain the research process. The researcher requested and receive the list of the eligible residents from the directors of all three nursing homes. Also, a list of RNs who work with these eligible residents was obtained. 
Demographic description. After obtaining informed consent, the researcher collected demographics such as age, gender, and length of stay from the residents' medical records.

\section{Data collection.}

Change tracking forms. Following consent, the researcher met with nurses' individually or in small groups to train them in completing the Tracking Forms and CON-DAT. The researcher showed the nurses samples of the Tracking form and CON-DAT tool and explain in detail each item of the form and measurement tool. The researcher filled out a tracking form and CON-DAT in front of the nurses to demonstrate how the forms and CON-DAT should be filled out. The researcher answered all questions that the nurses asked during the training session.

To extend the orientation, the researcher was present in each nursing home on the first day of data collection welcoming both nurses and clients and distributing the tools. Eligibility criteria were re-checked and un-eligible subjects were removed. An offer was made to read all questionnaire items and provide assistance filling out the forms. All study procedures took place in a quiet, private room with adequate lighting and a flat writing surface. Upon completion, the researcher checked all data collection sheets for missing data. The researcher was available to assist both nurses and residents throughout the study if they might have questions about data collection.

The researcher asked nurses to start filling Change Tracking forms (Appendix E) once they noticed change in residents' behavior or condition. Nurses recorded their level of certainty that the resident had physical pain and discomfort as a result of changes prior to doing their assessment of suspected pain. Then the nurses conducted an assessment of pain determined by their initial certainty level. After that, nurses recorded again their level of certainty the resident has physical pain and discomfort. 
Pain and agitation outcomes. Pain and agitation outcomes were measured by the researcher and research assistant using the DS-DAT and WAI respectively. The researcher trained a research assistant to administer the DS-DAT and WAI tools and check for completeness. An attempt was made to complete any missing items. In 3 and 7 days after change in residents' condition, the researcher or research assistant measured the residents' level of pain. This timing of measures for pain was used to capture both short and longer-term changes, while keeping the study feasible and avoiding some other confounding events that could occur if a longer time period was used. Both measurements were done midday because it was more convenient time for both residents and nurses. Since it is unethical to insist nurses to complete pre-assessment level of certainty before doing their assessment of suspect pain so they would complete the pre-assessment level of certainty by recalling. This recalling bias might lead to measurement error.

The researcher administered the measurement tools for pain and agitation following a stressful event. The observation of outcomes was done twice for pain and four times for agitation, based on suggestions by Kovach (Personal communication, 2015) who has extensive experience using both tools. The WAI tool needs a three minutes observation period following a 10-minute washout period from the stressful event. Therefore, two 3 minutes observations of agitation were done with at least 10 minutes apart. While waiting for 10 minutes to do the second observation of agitation, the researcher or research assistant administered D-DAT tool for pain. After doing the second observation, the researcher waited for 30 minutes to the second round of pain and agitation observations. 
Table 2: Data Analysis Plan

\begin{tabular}{|c|c|c|c|c|c|}
\hline $\begin{array}{l}\text { Research Questions } \\
\text { /Hypotheses }\end{array}$ & $\begin{array}{l}\text { Unit of } \\
\text { Analysis }\end{array}$ & Variable & $\begin{array}{l}\text { Measureme } \\
\text { nt tool }\end{array}$ & $\begin{array}{l}\text { Level of } \\
\text { Measurement }\end{array}$ & $\begin{array}{l}\text { Statistical } \\
\text { Test }\end{array}$ \\
\hline \multicolumn{6}{|l|}{ Descriptive Questions: } \\
\hline $\begin{array}{l}\text { 1. What is the level of nurses' certainty about } \\
\text { suspected pain in nursing home residents with } \\
\text { dementia in Jordan before and after assessment? }\end{array}$ & Residents & DV: nurses' certainty & -Likert scale & -Ordinal & $\begin{array}{l}\text { Descriptive } \\
\text { (frequency, } \\
\text { mean, SD, }\end{array}$ \\
\hline $\begin{array}{l}\text { 3. Is there a difference in nurses' certainty of } \\
\text { suspected pain in residents who do and do not } \\
\text { verbally report symptoms? }\end{array}$ & Residents & $\begin{array}{l}\text { IV: ability to verbally } \\
\text { self report symptoms } \\
\text { DVs: Nurses' } \\
\text { certainty }\end{array}$ & $\begin{array}{l}\text {-CON-DAT } \\
\text {-Likert scale }\end{array}$ & $\begin{array}{l}\text {-Nominal } \\
\text {-Ordinal }\end{array}$ & T-test \\
\hline $\begin{array}{l}\text { 6. Is there a difference in nurses' use of } \\
\text { unidimensional and multidimensional assessment } \\
\text { between residents who do and do not verbally } \\
\text { report symptoms? }\end{array}$ & Residents & $\begin{array}{l}\text { IV: ability to verbally } \\
\text { self report symptoms } \\
\text { DV: Assessment } \\
\text { unidimensional or } \\
\text { multidimensional }\end{array}$ & $\begin{array}{l}-\mathrm{CON}-\mathrm{DAT} \\
\text {-Medical } \\
\text { record }\end{array}$ & $\begin{array}{l}\text {-Nominal } \\
\text {-Nominal }\end{array}$ & $\begin{array}{l}\text { Chi-square } \\
\text { test }\end{array}$ \\
\hline
\end{tabular}




\section{Primary Hypotheses}

(1) Certainty of suspected pain by the nurse will be associated with scope of pain assessment provided to a PWD who has a change in condition.

(2) Pre-assessment level of nurses' certainty, assessment scope, and post-assessment level of certainty will predict pain and agitation outcomes. Rationale: Step 3 of this model will test if, consistent with the RCP model, post assessment certainty is a unique significant predictor of resident outcomes.

(3) The relationship between pre-assessment level of nurses' certainty and outcomes is mediated by assessment scope. Rationale: Step 3 of this model will test mediating effects that are somewhat contrary to the RCP model by hypothesizing that it is assessment scope regardless of level of certainty that drives outcomes.

\begin{tabular}{|l|l|l|l|l|} 
Residents & $\begin{array}{l}\text { IV: nurses' certainty } \\
\text { DV: Assessment } \\
\text { scope }\end{array}$ & $\begin{array}{l}\text {-Likert scale } \\
\text {-Medical } \\
\text { record }\end{array}$ & $\begin{array}{l}\text {-Ordinal } \\
\text {-Ordinal }\end{array}$ & $\begin{array}{l}\text { Pearson r } \\
\text { correlation }\end{array}$ \\
\hline Residents & $\begin{array}{l}\text { IVs: Assessment } \\
\text { scope, Pre and post } \\
\text { assess certainty } \\
\text { DVs: pain/ Agitation }\end{array}$ & $\begin{array}{l}\text {-Medical } \\
\text { Record } \\
\text {-Likert scale } \\
\text {-D-DAT } \\
\text {-WAI }\end{array}$ & $\begin{array}{l}\text {-Ordinal } \\
\text {-Ordinal } \\
\text {-Ordinal } \\
\text {-Ordinal }\end{array}$ & $\begin{array}{l}\text { Hierarchical } \\
\text { regression }\end{array}$ \\
\hline Residents & $\begin{array}{l}\text { IVs: Assessment } \\
\text { scope, preassessment } \\
\text { level of certainty } \\
\text { DVs: pain/ Agitation }\end{array}$ & $\begin{array}{l}\text {-Medical } \\
\text { Record } \\
\text {-D-DAT } \\
\text {-WAI } \\
\text {-Likert scale }\end{array}$ & $\begin{array}{l}\text {-Ordinal } \\
\text {-Ordinal }\end{array}$ & $\begin{array}{l}\text { Hierarchical } \\
\text { regression }\end{array}$ \\
& & & & \\
\hline
\end{tabular}

Secondary Hypotheses: based on subgroup of residents treated by a nurse who has an initial high level of certainty of suspected pain:

(1) The relationship between assessment scope and outcomes will be mediated by post assessment level of certainty.

\begin{tabular}{|l|l}
\hline Residents & IVs: Nurses \\
& Certainty, \\
& Assessment Scope \\
& DVs: Pain \\
& Agitation
\end{tabular}

\begin{tabular}{l|l}
-Likert scale & -Ordinal \\
-Medical & -Ordinal \\
Record & -Ordinal \\
-D-DAT & -Ordinal \\
-WAI & \\
\hline
\end{tabular}

Hierarchical regression

Secondary Hypotheses: based on subgroup of residents treated by a nurse who has an initial low level of certainty of suspected pain

(2) Assessment scope and post-assessment certainty will predict outcomes.

\begin{tabular}{|l|l} 
Residents & IVs: Nurses \\
& Certainty, \\
& Assessment Scope \\
& DVs: Pain \\
& Agitation
\end{tabular}

Multiple

regression

\begin{tabular}{|l|l}
-Likert scale & -Ordinal \\
-Medical & -Ordinal \\
Record & -Ordinal \\
-D-DAT & -Ordinal \\
-WAI & \\
\hline
\end{tabular}




\section{Data Management Plan}

The researcher hired a research assistant and statistician to work all together on the data management plan. The selected statistician was qualified and did not diverge from the proposed plan developed by Dr. Kovach and Dr. Ke. Data were stored on a hard drive and backed up on the server of the researcher's computer. The computer is password protected. No identifying data were included in the stored computer data. The research stored hard copies of the completed data sheets in a locked file cabinet in a locked office. Only the researcher and research assistant had access to the locked office. The researcher and research assistant reviewed, double checked, and coded the data before entering them into SPSS file. Once at least same six sheets were completed by each of the researcher and research assistant, the inter-rater reliability was calculated to assess the consistency of the score measured. Inter-rater reliability was used to assess the degree to which different raters/observers give consistent estimates of the same phenomenon from the same participant (Polit \& Beck, 2012). In the following paragraphs, the data management plans will discuss in detail.

The researcher, research assistant, and statistician cleaned any missing data to ensure data accuracy. Missing data is a very common problem for many research endeavors, which may limit the credibility and generalizability of results sometimes (Palmer \& Royall, 2010). The plan for managing any potential missing data in this study was done by excluding observations with more than $10 \%$ missing data from the analysis, using case-wise or pair-wise deletion methods. Data cleansing is the process that helps in detecting and correcting inaccurate values that can result from entry process, which can affect the results (Palmer \& Royall).

When $25 \%$ of the data had been collected, data were checked to ensure that assumptions

for planned statistical analyses could be met. The researcher and research assistant checked all 
continuous variables for skew. Also, the researcher checked the scatterplots between each IV and DV for possible nonlinear relationships and any multicollinearity between IVs were checked. It is very important to check for multicollinearity because it may cause problems in interpreting the results. Multicollinearity increases the standard errors of the coefficients. It makes some variables statistically insignificant when they should be significant (Polit \& Beck, 2012). If problems arise, the statistical teams at the Jordan University of Science and Technology, the methodological experts, Dr. Kovach, and the researcher would work together to make decisions about any needed modifications. The researcher kept a log by tracing the history and rationale for any needed modifications.

\section{Data Analysis Plan}

Data analysis was conducted using the version 21 of SPSS, statistical software program. Descriptive statistics, t-test, one-way ANOVA and regressions were computed. An alpha level of 0.05 was used to determine statistical significance of all inferential tests used. The assumptions of each statistical test used were checked before running the tests. Even though the dependent variables were probably all ordinal, the interval level statistics were used if there is no severe skew. Therefore, the scatterplots between each IV and DV for possible nonlinear relationships and any multicollinearity between IVs were checked. Also, independence of observations was checked using the Durbin-Watson statistic, which is a simple test to run using SPSS Statistics.

Initially, the distributions of each dependent and independent variables were analyzed using frequency distributions, means, and standard deviations. Transformations were used in the presence of skewed distributions. Descriptive statistics were used to describe and summarize sample characteristics. Continuous variables such as age and length of stay were described using 
the mean and standard deviation. Categorical variables such as gender and race were described using frequencies, frequency distributions and percentages.

The data analysis plan for questions and hypotheses is summarized in Table 2. The first research question about the level of nurses' certainty was answered using descriptive data analysis including frequencies, means, and standard deviations. Means and standard deviations were calculated for each individual item and for each of the five constructs or categories. The second question was answered using one-way ANOVA to see if there is a difference in nurses' certainty of suspected pain when they care for residents with mild, moderate or severe dementia. T-test analysis was used to see if there is a difference in nurses' certainty of suspected pain when they care for residents who do or do not verbally report symptoms. The fourth question described frequencies of unidimensional and multidimensional assessment. Chi square tests was used to answer both the fifth and sixth questions, as all variables are nominal level.

To test the first main hypothesis, Pearson correlation was used to analyze the effect of level of nurses' certainty on the assessment scope (whether is unidimensional or multidimensional) provided to a PWD who has a change in condition. To test the second main hypothesis, hierarchical regression was used to examine the effect of the key independent variable. In this analysis, pre-assessment level of nurses' certainty variable was entered into the model first, then assessment scope variable, and post-assessment level of nurses' certainty variable was entered last. This order of variables is consistent with the RCP model. The third step of this model tested if post assessment certainty is a unique significant predictor of resident outcomes.

The third main hypothesis was analyzed using hierarchical regression to test the mediating effect of assessment scope on the relationship between pre-assessment level of nurses' 
certainty and pain and agitation level in PWD. The third step of this model tested mediating effects that are somewhat contrary to the RCP model by hypothesizing that it is assessment scope regardless of level of certainty that drives outcomes.

The secondary hypotheses involve subgroups from the main sample and are exploratory. Based on the subgroup of residents treated by a nurse who has an initial high level of certainty of suspected pain, hierarchical regression analysis was used to test the mediating effect of post assessment level of certainty on the relationship between assessment scope and outcomes in PWD after controlling for pre-assessment level of certainty. Next, based on the subgroup of residents treated by a nurse who has an initial low level of certainty of suspected pain, two separate multiple regressions analyzed the effect of assessment scope and post-assessment certainty on the level of pain and agitation in PWD after controlling for pre-assessment level certainty.

\section{Limitations}

This study has limitations associated with the study design, sample size, sampling method, measurement, and extraction information about the clients. The first limitation was the use of a descriptive, correlational design that does not permit causality to be inferred. Moreover, according to Polit and Beck (2012), nonexperimental studies are the weakest ones in comparison with experimental or quasi-experimental research. Second, the use of self-report method to collect data could increase the possibility of recall bias among the participants. Third, sample size might also be another limitation since the study was limited to one geographical area, which might cause low participation rate and limit the generalizability of the findings to other nursing home residents from different geographical area. Fourth, the non-probability convenience sampling method of this study might introduce selection bias to the internal validity of the study 
(Polit \& Beck). Also, the convenience sampling method used in this study might limit the generalizability of findings. Probability sampling method would be too costly to administer and unfeasible. Measurement error can occur in any study. To minimize measurement error, the researcher and research assistant consistently did interrater reliability checks for every $20 \%$ of data collection. If interrater reliability was $>.85$, the researcher would approve that the data collected in the study are correct representations of the variables measured.

Another limitation could be the low response rate and incomplete filling the questionnaires or the tools due to difficulty getting the information about the participants. One reason might be due to unstructured filing system of home visiting clients due to the new application of nursing homes in Jordan. Uncontrolled variables that contributed to the pain and agitation levels could also be a limitation of this study. These uncontrolled variables may include different cultural background or religious affiliation. Using only complete data and excluding missing data in the analysis may degrade the final analytical sample, reducing power and causing selection bias. Finally, since several PWD are cared for by the same nurse, it might be difficult for nurses to recall clearly all needed information about each resident. This recall bias could affect the reliability of self-report data from nurses and in turns may influence the results. 


\section{Chapter Summary}

The goal of this descriptive, correlational study was to fill gaps currently existing in available literature. This chapter provided an overview of the sample, procedures for data collection, and instrumentation employed in this study. Also it provides a review of data analysis techniques used in this study. Finally, this chapter included the identified research design limitations in this study. 


\section{CHAPTER 4}

This chapter includes the third manuscript required for this dissertation. This paper is the research study manuscript and describes the sample and presents the statistical results for all research questions and hypotheses. Also this chapter includes a study report of additional research questions and hypotheses about other variables because of the very high percentages of variance accounted for by a limited number of variables in the original study.

\section{Manuscript 3: The Association of Nurses' Assessment and Certainty to Pain Management and Outcomes for Nursing Home Residents in Jordan \\ Introduction to the Problem}

Pain is one of the most prevalent problems in the older adult population. Nearly $80 \%$ of older adults living in nursing homes $(\mathrm{NH})$ and up to 50\% living at home suffer pain (Lukas et al., 2012). People with dementia (PWD) are at even greater risk for unrelieved pain (Lukas et al.). Studies have consistently shown the high prevalence of poor assessment and inadequate treatment of pain in NH residents with dementia (Reynolds, Hanson, DeVellis, Henderson, \& Steinhauser, 2008; Williams, Zimmerman, Sloane, \& Reed, 2005; Wu, Miller, Lapane, Roy, \& Mor, 2005). In one study of NH residents a researcher found that nearly $65 \%$ of residents with dementia had chronic pain; however, the nurses caring for them had detected less than $50 \%$ of these painful conditions (Huffman \& Kunik, 2000).

PWD often have both cognitive and communication problems that make them unable to verbally self-report their symptoms and comfort needs clearly (Kovach, Noonan, Griffie, Muchka, \& Weissman, 2001). It is challenging to assess pain in PWD if nurses misunderstand the needs or misinterpret the behaviors of PWD (Horgas \& Miller, 2006). Consequently, nurses may become uncertain regarding suspected pain in PWD and fail to provide timely treatment of 
pain or even any treatment at all (Gilmore-Bykovskyi \& Bowers, 2013). Unrelieved pain in PWD can lead to serious negative health consequences, such as agitation, depression, weight loss, dehydration, sleep and activity pattern disturbance, functional and cognitive impairment, and prolonged hospitalization (Horgas \& Miller; Kovach et al.).

\section{Background}

Uncertainty regarding suspected pain may be a major barrier to adequately assess and treat pain in nursing homes throughout the world (Almomani, McDowd, Bani-Issa, \& Almomani, 2014: Gilmore-Bykovskyi \& Bowers, 2013). Although a first line treatment for pain is often pharmacological, nurses who are uncertain regarding suspected pain in PWD may delay effective treatment by using additional decision-making steps or may not provide any pain relief at all (Gilmore-Bykovskyi \& Bowers). Theory and evidence suggest that uncertainty regarding suspected pain in PWD is primarily caused by: (1) under-recognition, misunderstanding, and misinterpreting of behavior changes in PWD by nurses; (2) complexity of pain assessment due to inconsistency of behavior changes; (3) inability of PWD to clearly communicate with nurses (Kaasalainen et al., 2007; Shega, Hougham, Stocking, Cox $\square$ Hayley, \& Sachs, 2006; Sheilds et al., 2013).

Gilmore-Bykovskyi and Bowers (2013) developed the Responses to Certainty of Pain (RCP) model to describe the concept of nurses' certainty regarding suspected pain and how it relates to the problem of underassessment and under-treatment of pain in PWD. This model provides an understanding of the decision making process that nurses employ when caring for PWD in pain. The RCP model is the first model to posit relationships between nurses' level of pain certainty, scope of pain assessment, and health outcomes of NH residents with dementia. When a person with dementia has a change in behavior or condition, nurses may try out several 
critical thinking and decision-making trajectories (Chang, Oh, Park, Kim, \& Kil, 2011; GilmoreBykovskyi \& Bowers, 2013).

The RCP model (Figure 1) describes two trajectories that are hypothesized to occur when a nurse has either a high or a low level of certainty of suspected pain. Both trajectories have three possible decision-making branches. The RCP model explains that when a nurse has a high level of certainty of suspected pain, the nurse follows the Response to Certainty Trajectory of decisions: in the first decision-making branch, the nurse decides to conduct a unidimensional assessment rather than a multidimensional assessment; in the second decision-making branch, if the unidimensional assessment leads to a high level of certainty of pain, the nurse decides to provide analgesics in a timely manner and the person with dementia will have better outcomes; in the third decision-making branch, if the unidimensional assessment leads to a low level of certainty of pain, the nurse decides to delay or forgo treatment and the person with dementia will have worse outcomes.

In the RCP model, when a nurse has a low level of certainty of suspected pain, the nurse follows the Response to Uncertainty Trajectory of decisions: (1) the nurse decides to conduct a multidimensional assessment or uses a trial and error approach. (2) if the multidimensional assessment leads to a high level of certainty of pain, the nurse decides to provide analgesics and the person with dementia will have better outcomes. (3) if the nurse decides to use a trial and error approach, the nurse will forgo treatment of pain and, the person with dementia will have worse outcomes. 
Figure 1: The Response to Certainty Model

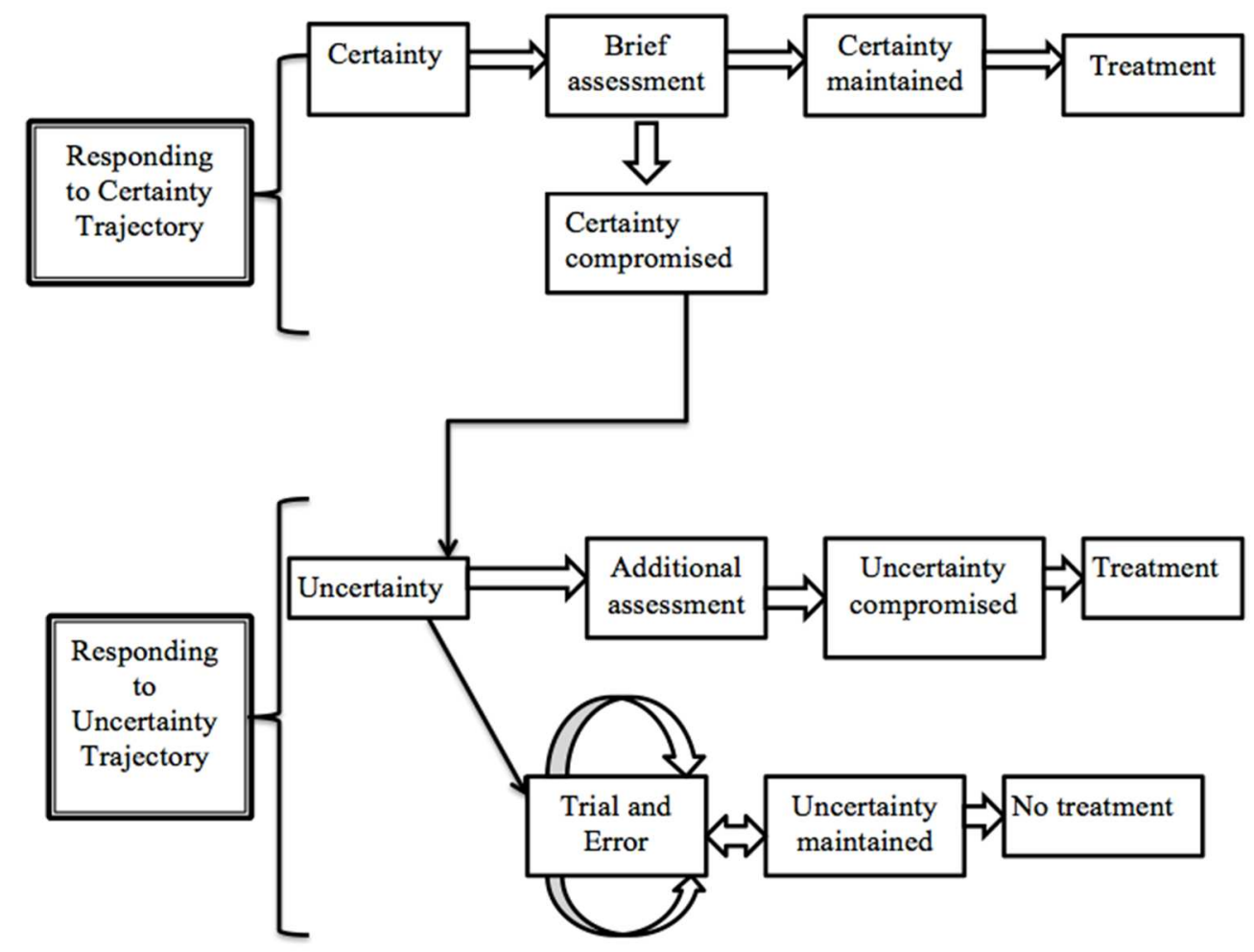

The RCP model has never been tested empirically. No previous study has directly examined the relationships between nurses' level of pain certainty, assessment scope, and patient outcomes. Therefore, the purpose of the study is to test the RCP model by examining the relationships between nurses' level of pain certainty, scope of pain assessment, and health outcomes of NH residents with dementia. All hypotheses and questions of the study are outlined in Table 1.

\section{Table 1: Research Questions and Hypotheses}

\section{Research Questions}

1. What is the level of nurses' certainty about suspected pain in nursing home residents with dementia in Jordan before and after assessment?

2. Is there a difference in nurses' certainty of suspected pain between residents with mild, moderate and severe dementia?

3. Is there a difference in nurses' certainty of suspected pain in residents who do and do not verbally report symptoms? 
4. What is the frequency of using unidimensional and multidimensional assessment?

5. Is there a difference in nurses' use of unidimensional and multidimensional assessment between residents with mild, moderate and severe dementia?

6. Is there a difference in nurses' use of unidimensional and multidimensional assessment between residents who do and do not verbally report symptoms?

\section{Research Primary Hypotheses}

(4) Certainty of suspected pain by the nurse will be associated with scope of pain assessment provided to a PWD who has a change in condition.

(5) Pre-assessment level of nurses' certainty, assessment scope, and post-assessment level of certainty will predict pain and agitation outcomes. Rationale: Step 3 of this model will test if, consistent with the RCP model, post assessment certainty is a unique significant predictor of resident outcomes.

(6) The relationship between pre-assessment level of nurses' certainty and outcomes is mediated by assessment scope. Rationale: Step 3 of this model will test mediating effects that are somewhat contrary to the RCP model by hypothesizing that it is assessment scope regardless of level of certainty that drives outcomes.

\section{Research Secondary Hypotheses}

(3) The relationship between assessment scope and outcomes will be mediated by post assessment level of certainty.

(4) Assessment scope and post-assessment certainty will predict outcomes.

\section{METHODS}

\section{Research Design, Sample, Setting}

A descriptive-correlational design was used in this study, which was conducted in five nursing homes in Amman, Jordan. A convenience sample of NH residents who have dementia and known pain or a known pain diagnosis were included in the study and yielded a sample size of 76. The inclusion criteria of the study were: (1) NH residents who are aged 55 and above. (2) NH residents with known pain or a known pain diagnosis. (3) NH residents whose family members or caregivers live in the same geographical area of the study in Jordan, Amman. (4) NH residents with any degree of memory loss or cognitive impairment as documented in medical charts or reported by nurses in case medical charts are incomplete. (5) NH residents who are cared by nurses with a minimum of one-year experience in nursing homes and working full time (at least 30 hours a week). The eligibility criteria were discussed with nursing staff and all 
eligible participants were included. This study was approved by the Institutional Review Board (IRB) of the University of Wisconsin-Milwaukee (UWM) and the designated IRB for each site. Written informed consent was obtained from the participants. For those NH residents who were unable to make their own decision, the power of attorney was used. All of the information collected was de-identified and securely stored.

\section{Measurements}

Outcome measures. Observational visual analog tools were used to measure pain (Discomfort-DAT) (Hurley, Volicer, Hanrahan, Houde, \& Volicer, 1992) and agitation (Wisconsin Agitation Inventory (WAI) (Kovach et al., 2004). The Discomfort-DAT, with a possible range of 0 to 75 , requires a 5 -minute observation period to measure the overall level of pain and the WAI, with a possible range of 0 to 100 , measures the number, duration and intensity of 30 behaviors through multiple 3-minute observations. Items in the Discomfort-DAT assess facial expression, body tension, fidgeting, and negative vocalization. Examples of agitated behaviors included in the WAI are hitting, pacing, repetitive questioning, and verbal combativeness. Internal consistency alpha coefficients ranged between .76 and .88 for the Discomfort-DAT third day measurements and between .73 and .87 for the seventh day measurements. Interrater reliability was achieved for both the Discomfort-DAT and WAI (kappa .90 and .93 respectively), and retested every $10 \%$ of data collection to prevent measurement error. Both the Discomfort-DAT and WAI have been responsive to change in previous intervention studies (Kovach, Logan, et al., 2006; Kovach et al., 2004).

Predictor measures. Predictor measures were extracted from tracking forms completed by the nurses during the data collection period. The tracking forms included areas for recording changes in behavior or condition, pain assessment, and nurses' certainty. Nurses were taught to 
complete change tracking using case studies until interrater reliability was .85 or greater. In order to assure valid and complete tracking of information, the tracking forms were crosschecked with the nurses' reports and resident medical records to investigate and resolve discrepancies.

Nurses' certainty was measured with a 1-item scale (score1-5) with the following responses: "very certain," "certain," "don't know," "uncertain," and "very uncertain." Assessment scope was measured by reviewing the nurses' tracking form assessment notes to identify the range of tools and types of assessments nurses used to determine their patients' pain levels, from no assessment to behavioral and functional assessment. When assessment scope was treated as an ordinal variable, it was coded based on the following coding rubric: $0=$ no assessment; 1 = brief self-report; 2 = numerical scale; $3=$ face or color scale; $4=$ behavioral assessment (with or without self-report); 5 = functional assessment (with or without self-report); $6=$ combined behavioral and functional assessment (with or without self-report). When assessment scope was treated as a nominal variable, it was coded as: $0=$ Unidimensional assessment (includes scores of 1-3 from the previous coding rubric), and 1= multidimensional assessment (includes scores of 4-6). Interrater reliability by two raters for assessment scope was 80.

Other measures. The Mini Mental Status Exam (MMSE) was used to measure the level of dementia. It consists of 30 questions, measuring orientation, registration, recall, attention, calculation, and language (Ellis, 2006). The range of scores is 0-30. Higher scores indicate greater cognitive ability. The Cumulative Illness Rating Scale for Geriatrics (CIRS-G) was used to measure Comorbid burden (Lin et al., 1968). The scale captures 15 of the more prevalent clinical conditions that are graded on 0-4 disease severity scale to yield ordinal level data (Borson et al., 2010). The CIRS-G was administered one time following consent. The 
Communication of Need-Dementia Alzheimer's Type (CON-AD) tool was used to measure the ability of PWD whether or not still to verbally self-report symptoms (Kovach \& Venes, 2003).

\section{Procedures}

Nurses received 7 hours of instruction from the researcher regarding: a) filling out the Change Tracking sheet; and b) administering the CON-DAT tool. Information provided during the training included common behavior changes associated with pain (e.g., facial grimacing, restless body movement) as well as changes in behavior that are less commonly associated with pain (e.g., change in appetite, decreased interest in activities). Four case study booklets were used to train and test the accuracy of filling out the tracking forms. Further training and retesting were done when accuracy was less than $80 \%$.

Following the training session, the tracking form sheets were initiated when a participant had changes in behavior or condition. In the tracking forms nurses recorded their level of certainty that the participant had physical pain and discomfort as a result of changes prior to doing their assessment of suspected pain. Then the nurses conducted an assessment of pain determined by their initial certainty level. After that, nurses recorded again their level of certainty that the resident has physical pain and discomfort. The researcher met with the nurses twice a week to answer their questions. The researcher was also contacted by phone several times by nurses when particularly challenging questions arose. To assure valid and complete tracking of information, the tracking forms were crosschecked with the nurses' reports and resident medical records to investigate and resolve discrepancies. In 3 and 7 days after a change in the residents' condition, the researcher or research assistant measured the residents' level of pain and agitation. Both measurements were done midday following a stressful event. The observation of outcomes was done twice for pain and four times for agitation, based on 
suggestions by Kovach (Personal communication, 2015) who has extensive experience using both tools.

\section{Data Analysis}

The SPSS 20 for Windows (SPSS Inc., Chicago, IL, USA) was used for the statistical analyses. The assumptions of normal distribution, homogeneity of variances, and linearity and independence of data were tested and outliers were excluded. Descriptive statistics were used to describe nurses' level of certainty, assessment type, and demographic data. A chi-square test was used to compare for differences in nominal level variables. A one-way ANOVA was used to analyze for differences in nurses' certainty among the residents by the severity of dementia. The chi-square test was used to analyze for differences in nurses' use of assessment type among the residents by severity of dementia and ability to report symptoms. The t-test was used to analyze for differences in nurses' certainty between residents who could and could not verbally report symptoms. A Pearson correlation was used to test the first primary hypothesis. A hierarchical regression method was used to examine the significant predictors of residents' outcomes and mediating effects of variables. Tolerance values of less than 0.1 for independent variables indicated that multicollinearity was not present. In hierarchical multiple regression equations the order of entry of the variables is important. Since this study is all about testing the RCP model, the order of entry the variables was based on their temporal order in the RCP model. Postassessment nurses' level of certainty is the main dependent variable and was entered into the regression models in the final step. The order of entry into the model yielded the variability in the levels of pain and agitation that was uniquely accounted for by Postassessment nurses' level of certainty. A Cronbach's alpha test was run for each time point when the Discomfort scale was administered. Cronbach alpha reliability indices for the Discomfort-DAT ranged from .89 to .92. 
Cronbach alpha reliability for the CON-DAT was .94. Interrater reliability for items in 10 Discomfort-DAT's and 10 WAI's were .88 and .89 respectively.

\section{Result}

\section{Description of Participants}

The mean age for the 76 residents was 72.52 years (SD 9.45, range 55-95) and 56.0\% were men. The average length of stay for the residents was 41.7 months (SD 10.33, range 3593). Twenty-two percent $(n=17)$ of the residents had severe dementia; the mean MMSE score was 14.44 (SD 6.54, range $0-23$ ). Nurses were certain regarding suspected pain in $23.6 \%$ of 76 PWD before assessment and 33\% $(n=76)$ after assessment. In this sample, $68 \%$ of nurses $(n=$ 10) used multidimensional assessment of pain in PWD.

\section{Comparisons based on Level of Dementia and Communication Deficit}

The 76 residents were divided into three groups according to severity of dementia: Severe $(\mathrm{MMSE}<10, \mathrm{n}=17 ; \mathrm{M}=2.1, \mathrm{SD}=1.01)$, moderate $(11>\mathrm{MMSE}<18, \mathrm{n}=41 ; \mathrm{M}=2.85, \mathrm{SD}=1.09)$ and mild $(19>\mathrm{MMSE}<25, \mathrm{n}=18 ; \mathrm{M}=3.32, \mathrm{SD}=1.10)$ for comparison with the associated scores of nurses on the Likert scale of certainty before and after assessment. Analysis with oneway ANOVA indicated a statistically significant difference among the groups $\left(\mathrm{F}_{(2,75)}=4.34, \mathrm{p}=\right.$ $0.017)$ and $\left(\mathrm{F}_{(2,75)}=3.94, \mathrm{p}=0.027\right)$ respectively. As PWD become severely demented, their nurses become less certain regarding suspected pain. Also, the 76 residents with dementia were divided into two groups according to their ability to verbally self-report symptoms: able to selfreport $(n=50 ; M=3.03, S D=1.12)$ and unable to self-report $(n=26 ; M=2.20, S D=.95)$ for comparison with the associated scores of nurses on the Likert scale of certainty before and after assessment. Analysis with t-test indicated a statistically significant difference among the groups $\left(\mathrm{t}_{74}=3.31, \mathrm{p}=0.002\right)$ and $\left(\mathrm{t}_{74}=2.22, \mathrm{p}<0.029\right)$ respectively. Based on these analyses, nurses 
were less certain regarding suspected pain in PWD who are unable to self-report pain.

A chi-square test was performed to examine the relationship between the pain assessment type and the ability to verbally self-report pain. The relation between these variables was significant, $\mathrm{X}^{2}(1, \mathrm{~N}=75)=9.80, \mathrm{p}<.05$. PWD who were unable to self-report pain were less likely to get a unidimensional assessment than those who were able. A chi-square test was also performed to examine the relationship between pain assessment type and severity of dementia. The relationship between these variables was significant, $\mathrm{X}^{2}(2, \mathrm{~N}=75)=8.68, \mathrm{p}<.01$. As PWD became severely demented, they were less likely to receive a unidimensional assessment and more likely to receive a multidimensional assessment.

\section{Primary Hypotheses}

Primary hypothesis one. Bivariate correlation was used to examine the association between the certainty of suspected pain and the assessment scope. The certainty of suspected pain by the nurse and the scope of pain assessment provided to a PWD were moderately negatively correlated, $\mathrm{r}=-.554, \mathrm{p}<.001$. As the level of dementia became more severe, the scope of assessment increased.

Primary hypothesis two. Tables 1-3 describe the relative contribution of pre assessment level of nurses' certainty, assessment scope, and post assessment level of nurses' certainty as predictors of agitation and pain outcomes. Primary hypothesis two was supported. As seen in Tables 1-3, at Step 1 pre assessment level of certainty significantly predicted agitation and pain 1 and pain 2 scores. The percentage of variance accounted for was $40 \%$ for agitation, $41.5 \%$ for pain 1 and $41.9 \%$ for pain 2 . At step 2 assessment scope was a significant predictor for all outcomes and added $1.9 \%$ of the variance to agitation, $1.2 \%$ to pain 1 and $1 \%$ to pain 2 . At Step 3 post assessment level of nurses' certainty uniquely predicted a statistically significant 
percentage of variance in all outcomes $(22.5 \%$ for agitation, $20.8 \%$ for pain 1 and $19.1 \%$ for pain

2). Overall, pre assessment level of nurses' certainty, assessment scope and post assessment level of nurses' certainty accounted for $64 \%$ of the variance in agitation, $62 \%$ of the variance in pain 1 and $60 \%$ of the variance in pain 2.

Table 1. Summary of Hierarchical Regression Analysis with Agitation Scores as Criterion $(\mathbf{N}=\mathbf{7 6})$.

\begin{tabular}{lccccc}
\hline Step and Predictor Variable & $\mathrm{R}^{2}$ & $\Delta \mathrm{R}^{2}$ & $\beta$ & $\mathrm{t}$ & $\mathrm{p}$ \\
\hline Step 1: & & & & & \\
Pre Assessment level of nurses Certainty & .400 & $.400^{*}$ & -.638 & -7.182 & $<.001^{*}$ \\
Step 2: & & & & & \\
Pre Assessment level of nurses Certainty & & & -.541 & -5.312 & $<.001^{*}$ \\
Assessment Scope & .419 & .019 & .190 & 1.865 & .066 \\
Step 3: & & & & & \\
Pre Assessment level of nurses Certainty & & & -.276 & -3.182 & $.002^{*}$ \\
Assessment Scope & & & .185 & 2.357 & $.021^{*}$ \\
Post Assessment level of nurses Certainty & .644 & $.225^{*}$ & -.577 & -7.160 & $<.001^{*}$ \\
$F_{(3,72)}=48.86, p<.0001$ & & & & & \\
\hline$* p<.05$ & & & & &
\end{tabular}

Table 2. Summary of Hierarchical Regression Analysis with Pain 1 Scores as Criterion (N $=76)$.

\begin{tabular}{lccccc}
\hline Step and Predictor Variable & $\mathrm{R}^{2}$ & $\Delta \mathrm{R}^{2}$ & $\beta$ & $\mathrm{t}$ & $\mathrm{p}$ \\
\hline $\begin{array}{l}\text { Step 1: } \\
\text { Pre Assessment level of nurses Certainty }\end{array}$ & .415 & $.415^{*}$ & -.644 & -7.30 & $<.01^{*}$ \\
Step 2: & & & & & \\
Pre Assessment level of nurses Certainty & & & -.618 & -5.98 & $<.001^{*}$ \\
Assessment Scope & .427 & .012 & .051 & .492 & .624 \\
Step 3: & & & & & \\
Pre Assessment level of nurses Certainty & & & -.365 & -3.98 & $<.001^{*}$ \\
Assessment Scope & & & .046 & .561 & .577 \\
Post Assessment level of nurses Certainty & .635 & $.208^{*}$ & -.529 & -6.51 & $<.001^{*}$ \\
$F_{(3,72)}=41.63, p<.0001$ & & & & & \\
\hline$* p<.05$ & & & & &
\end{tabular}

Table 3. Summary of Hierarchical Regression Analysis with Pain 2 Scores as Criterion (N $=76)$.

\begin{tabular}{lccccc}
\hline Step and Predictor Variable & $\mathrm{R}^{2}$ & $\Delta \mathrm{R}^{2}$ & $\beta$ & $\mathrm{t}$ & $\mathrm{p}$ \\
\hline Step 1: & & & & & \\
Pre Assessment level of nurses Certainty & .419 & $.419^{*}$ & -.653 & -7.474 & $<.001^{*}$ \\
Step 2: & & & & & \\
Pre Assessment level of nurses Certainty & & & -.658 & -6.417 & $<.001^{*}$ \\
Assessment Scope & .429 & .010 & .008 & -.083 & .734 \\
Step 3: & & & -.410 & -2.456 & $<.001^{*}$ \\
Pre Assessment level of nurses Certainty & .620 & $.191^{*}$ & .013 & .150 & .880 \\
\hline
\end{tabular}


Assessment Scope

$-.506-5.986<.001^{*}$

Post Assessment level of nurses Certainty

$F_{(3,72)}=38.97, p<.0001$

$* p<.05$

Primary hypothesis three. The notion that assessment scope mediates the relationship between pre assessment level of nurses' certainty and outcomes was not supported. As seen in the betas in Step 2 of Tables 4-6, when assessment scope was added to the models, the contribution of the nurses' certainty prior to assessment changed by only small amounts and remained statistically significant.

Table 4. Summary of Hierarchical Regression Analysis with Agitation Scores as Criterion $(\mathbf{N}=\mathbf{7 6})$.

\begin{tabular}{lccccc}
\hline Step and Predictor Variable & $\mathrm{R}^{2}$ & $\Delta \mathrm{R}^{2}$ & $\beta$ & $\mathrm{t}$ & $\mathrm{p}$ \\
\hline $\begin{array}{l}\text { Step 1: } \\
\text { Pre Assessment level of nurses Certainty }\end{array}$ & .400 & $.400^{*}$ & -.638 & -7.18 & $<.001^{*}$ \\
Step 2: & & & & & \\
Pre Assessment level of nurses Certainty & & & -.541 & -5.31 & $<.001^{*}$ \\
Assessment Scope & .419 & .019 & .190 & 1.87 & .066 \\
$F_{(2,73)}=28.38, p<.0001$ & & & & & \\
$* p<.05$ & & & & &
\end{tabular}

Table 5. Summary of Hierarchical Regression Analysis with Pain 1 Scores as Criterion (N $=76$ ).

\begin{tabular}{lccccc}
\hline Step and Predictor Variable & $\mathrm{R}^{2}$ & $\Delta \mathrm{R}^{2}$ & $\beta$ & $\mathrm{t}$ & $\mathrm{p}$ \\
\hline Step 1: & & & & & \\
Pre Assessment level of nurses Certainty & .415 & $.415^{*}$ & -.644 & -7.02 & $<.001^{*}$ \\
Step 2: & & & & & \\
Pre Assessment level of nurses Certainty & & & -.618 & -5.98 & $<.001^{*}$ \\
Assessment Scope & .427 & .012 & .015 & .492 & .624 \\
$F_{(2,73)}=26.46, p<.0001$ & & & & & \\
$* p<.05$ & & & & &
\end{tabular}

Table 6. Summary of Hierarchical Regression Analysis with Pain 2 Scores as Criterion (N $=76$ ).

\begin{tabular}{lccccc}
\hline Step and Predictor Variable & $\mathrm{R}^{2}$ & $\Delta \mathrm{R}^{2}$ & $\beta$ & $\mathrm{t}$ & $\mathrm{p}$ \\
\hline Step 1: & & & & & \\
Pre Assessment level of nurses Certainty & .419 & $.419^{*}$ & -.653 & -7.474 & $<.001^{*}$ \\
Step 2: & & & & & \\
Pre Assessment level of nurses Certainty & & & -.658 & -6.417 & $<.001^{*}$ \\
Assessment Scope & .429 & .010 & .008 & -0.83 & .73 \\
$F_{(2,73)}=27.56, p<.0001$ & & & & & \\
\hline$* p<.05$ & & & & &
\end{tabular}




\section{Secondary Hypotheses}

Secondary hypothesis one. The hypothesis that certainty after assessment will mediate the relationship between assessment scope and outcomes was supported. It is noteworthy that at Step 1 assessment scope accounted for a large percentage of variance in agitation, pain 1 and pain 2 outcomes $(30.6 \%, 14.4 \%$ and $15 \%$, respectively). However, as seen in Tables $7-9$, the betas for assessment scope changed from significant in Step 1 to not significant in Step 2 when post assessment level of certainty was added to the model.

Table 7. Summary of Hierarchical Regression Analysis with Agitation Scores as Criterion $(\mathbf{N}=19)$.

\begin{tabular}{lccccc}
\hline Step and Predictor Variable & $\mathrm{R}^{2}$ & $\Delta \mathrm{R}^{2}$ & $\beta$ & $\mathrm{t}$ & $\mathrm{p}$ \\
\hline Step 1: & & & & & \\
Assessment Scope & .306 & $.306^{*}$ & .585 & 3.06 & $.007^{*}$ \\
Step 2: & & & & & \\
Assessment Scope & & & .362 & 2.205 & .061 \\
Post Assessment level of nurses Certainty & .559 & $.253^{*}$ & -.567 & -3.46 & $.003^{*}$ \\
$F_{(2,16)}=13.54, p<.0001$ & & & & & \\
\hline$* p<.05$ & & & & &
\end{tabular}

Table 8. Summary of Hierarchical Regression Analysis with Pain 1 Scores as Criterion (N =19).

\begin{tabular}{lccccc}
\hline Step and Predictor Variable & $\mathrm{R}^{2}$ & $\Delta \mathrm{R}^{2}$ & $\beta$ & $\mathrm{t}$ & $\mathrm{p}$ \\
\hline Step 1: & & & & & \\
Assessment Scope & .144 & $.144^{*}$ & .435 & 2.05 & $.054^{*}$ \\
Step 2: & & & & & \\
Assessment Scope & & & .150 & .932 & .364 \\
Post Assessment level of nurses Certainty & .547 & $.403^{*}$ & -.723 & -4.501 & $.001^{*}$ \\
$F_{(2,16)}=14.477, p<.0001$ & & & & & \\
\hline$*<<.05$ & & & & &
\end{tabular}

Table 9. Summary of Hierarchical Regression Analysis with Pain 2 Scores as Criterion (N =19).

\begin{tabular}{lccccc}
\hline Step and Predictor Variable & $\mathrm{R}^{2}$ & $\Delta \mathrm{R}^{2}$ & $\beta$ & $\mathrm{t}$ & $\mathrm{p}$ \\
\hline Step 1: & & & & & \\
Assessment Scope & .150 & $.150^{*}$ & .442 & 3.08 & $.001^{*}$ \\
Step 2: & & & & & \\
Assessment Scope & & & .147 & .963 & .349 \\
Post Assessment level of nurses Certainty & .620 & $.470^{*}$ & -.740 & -4.86 & $.001^{*}$ \\
$F_{(2,16)}=16.744, p<.0001$ & & & & & \\
\hline$* p<.05$ & & & & &
\end{tabular}


Secondary hypothesis two. A multiple linear regression was calculated to predict patients' outcomes based on assessment scope and post-assessment certainty when nurses have an initial low level of certainty of suspected pain. As seen in Table 10-12, a significant regression equation was found (pain $1 ; \mathrm{F}_{(2,54)}=14.53, \mathrm{p}<.0001$, with an $\mathrm{R}^{2}$ of .326), (pain $2 ; \mathrm{F}$ $(2,54)=17.14, \mathrm{p}<.0001$, with an $\mathrm{R}^{2}$ of .366), and (agitation; $\mathrm{F}_{(2,54)}=22.04, p<.0001$, with an $\mathrm{R}^{2}$ of .429). Post assessment level of nurses' certainty is statistically significant while the beta for assessment scope is insignificant in the level of pain after 7 days $(\mathrm{p}=.404)$, and the level of pain after 3 days $(\mathrm{p}=.370)$. This result indicates that the differences in the level of pain after 3 days, and the level of pain after 7 days in PWD were mainly dependent on post assessment level of nurses' certainty.

Table 10. Summary of Multiple Regression Analysis with Agitation Scores as Criterion (N =57).

\begin{tabular}{lcccc}
\hline Predictor Variable & $\mathrm{R} 2$ & $\beta$ & $\mathrm{t}$ & $\mathrm{p}$ \\
\hline Assessment Scope & & .262 & 2.592 & $.012^{*}$ \\
Post Assessment level of nurses Certainty & .429 & $-.602^{*}$ & -5.947 & $<.001^{*}$ \\
$\mathrm{~F}_{(2,54)}=22.04, p<.0001$ & & & & \\
\hline$* p<.05$ & & & &
\end{tabular}

Table 11. Summary of Multiple Regression Analysis with Pain1 Scores as Criterion ( $\mathbf{N}=$ 57).

\begin{tabular}{lcccc}
\hline Predictor Variable & $\mathrm{R} 2$ & \multicolumn{1}{c}{$\beta$} & $\mathrm{t}$ & $\mathrm{p}$ \\
\hline Assessment Scope & & .092 & .904 & .370 \\
Post Assessment level of nurses Certainty & .366 & $-.610^{*}$ & -5.72 & $<.001^{*}$ \\
$\mathrm{~F}(2,54)=17.14, \mathrm{p}<.0001$ & & & & \\
\hline$* p<.05$ & & & &
\end{tabular}

Table 12. Summary of Multiple Regression Analysis with Pain2 Scores as Criterion ( $\mathbf{N}=$ 57).

\begin{tabular}{lcccc}
\hline Predictor Variable & $\mathrm{R} 2$ & $\beta$ & $\mathrm{t}$ & $\mathrm{p}$ \\
\hline Assessment Scope & & .090 & .841 & .404 \\
Post Assessment level of nurses Certainty & .326 & $-.579 *$ & -5.266 & $<.001^{*}$ \\
$\mathrm{~F}_{(2,54)}=14.53, \mathrm{p}<.0001$ & & & & \\
\hline$* p<.05$ & & & &
\end{tabular}




\section{Discussion}

Our study tested the RCP model in four nursing homes in Jordan. All hypotheses about the relationship between nurses' certainty regarding suspected pain, scope of pain assessment, and health outcomes of NH residents with dementia were supported. These findings are overwhelmingly consistent with the RCP model, but conflict with findings from other research on pain in PWD in several critical and fundamental areas that will be discussed in this section. Findings in this study need to be interpreted with caution and replicated before being applied to practice or theory derivation.

This study also found relationships between the severity of dementia and the ability to self-report symptoms in PWD with nurses' certainty regarding suspected pain and scope of pain assessment. For example, the more severe the dementia and the less ability the residents had to verbally self-report symptoms, the more uncertain the nurses were regarding suspected pain and the less likely the nurses would be to use unidimensional assessment. In contrast, the less severe the dementia and the greater ability the residents had to verbally self-report symptoms, the more certain the nurses were regarding suspected pain and the more likely the nurses would be to use unidimensional assessment.

These findings about the severity of dementia, the ability to self-report symptoms, and nurses' use of unidimensional versus multidimensional assessment are consistent with previous studies (Gilmore-Bykovskyi \& Bowers, 2013; Hadjistavropoulos et al., 2012; Horgas et al., 2007; Lin et al.; Zwakhalen et al., 2007). Researchers have found that unidimensional assessment tools are still a reasonable and accurate way to assess pain in older adults with mild to moderate dementia (Herr, Coyne, McCaffery, Manworren, \& Merkel, 2011). Nurses caring for NH residents with severe dementia who are unable to verbally report pain struggle with 
recognizing their comfort needs. These nurses tend to use a multidimensional pain assessment tool to interpret observed behavioral changes (Herr et al., 2011). Nurses who are unable to establish their certainty regarding suspected pain might be stuck in a trial and error approach to treatment (Tait et al., 2009). Given the complexity of the pain cues in PWD, nurses tend to do additional pain assessment to establish certainty. The inability to discriminate between pain and dementia leaves nurses uncertain about whether behavioral changes are pain-related or dementiarelated (Kaasalainen et al., 2007).

The findings that support the hypotheses about predictors of residents' outcomes suggest that pre-assessment level of nurses' certainty, assessment scope, and post-assessment level of certainty are significant predictors. These results are consistent with studies that examined nursing assessment and studies that examined nurses' decision certainty and found these variables to be critical factors in improving nursing home residents' outcomes (Achterberg et al., 2013; Kovach, 2013). Achterberg et al. found that when healthcare providers are initially certain about pain in PWD, they tend to validate their certainty with additional assessments, such as interviewing family members, taking vital signs, or checking medical records. If certainty of pain in PWD is confirmed, nurses provide PWD with analgesics. However, when nurses are initially not certain about pain in PWD, they may start to think that the observed change in behavior is an aspect of dementia, rather than pain. In that case, the nurse may forgo gathering additional information and administering analgesics. Instead, nurses may respond to these behavioral changes in a way that does not address the resident's pain (Kaasalainen et al., 2007).

A study by Kaasalainen et al. (2007) found that the level of nurses' confidence about pain in PWD was significantly correlated with their self-assessment of pain. The finding of this study that shows nurses' certainty of suspected pain was associated with the scope of pain assessment, 
such as a self-report of pain, is consistent with Kaasalainen et al. findings and the RCP model.

This descriptive study, although profoundly limited, is the first study to explore the concept of certainty and how it relates to the problem of pain assessment and management in PWD. It is also the first study constructed to test the RCP model. It is also the first nursing research study in Jordan that was conducted in nursing home settings. Up until now, the limited number of nurse researchers who are working in Jordan focused their programs of research on hospital settings. Due to the preliminary nature of this study, the findings may not add to our understanding of pain management. However, this study does contribute to nursing science in so far as it highlights potential flaws in research design that could result in unreliable data when studying nurses' certainty of pain, especially in a Middle Eastern country such as Jordan. The flaws in this study that led to unreliable data include a number of unexpected uncontrolled confounding variables.

All the evidence of pain management science indicates that objective pain assessment by nurses and subjective self-report by patients are vitally important to patient outcomes. This study found that assessment scope does not mediate the relationship between pre-assessment certainty and patient outcomes among the nurse participants in the study. This finding contributes to nursing science by suggesting that if nurses' pre-assessment uncertainty does not change after assessment and patient outcomes do not improve after assessment, then there is something wrong with the way the nurses conducted the assessment of pain. An interventional study could be developed to test this explanation. In this kind of follow-up study nurses could be trained on assessment and certainty and patient outcomes could be measured in order to determine whether a lack of assessment skills contributed to this questionable finding.

Another way to understand this doubtful finding is to note that it is widely accepted in the 
scientific pain community that patients' signs and symptoms, as well as patients' self-reports, are reliable indicators for pain treatment and ensure better patient outcomes. Nurses are taught to do objective assessment of pain and to believe the subjective nature of a patient's self-report. The nurses in this study turned this well supported hierarchy of pain management upside down, which may explain the poor patient outcomes and lack of certainty. Exploring the reasons why these nurses turned this hierarchy upside down is a productive line of inquiry.

One reason for this finding may have stemmed from the fact that prior to the recent emergence of nursing homes in Jordan, PWD had been an unrecognized population since they were cared for in individual homes. Since the assessment of pain in PWD is complicated and because nurses in Jordan likely have a knowledge deficit about the mechanism of pain in PWD, nurses in this study may think that no matter how they care for their PWD they will still be in pain. If the nurse participants underestimated the ability of nurses to accurately assess and treat pain in PWD, they may have just gone through the motions of assessment for this study rather than skillfully employing assessment as a valuable nursing strategy. Therefore, a knowledge deficit about pain assessment in PWD could be an unexpected uncontrolled variable. A future study could include an evaluation of nurses' knowledge of pain in PWD in order to explore this variable's influence on the mediating effect of assessment scope.

A second reason for this finding may stem from a lack of nurses' autonomy in Jordanian nursing home settings. The inability to independently choose assessment scope could have added to the nurses' potential lack of engagement in the assessment process. In the three public nursing homes in this study it was noted that administrators, rather than nurses, determined the assessment scope to be routinely used with patients with severe dementia. Nurses had more autonomy in the two private nursing homes in this study to determine assessment scope on their 
own. However, in the private nursing homes it was noted in some instances that even if the nurse experienced certainty based on the patient's self-report of pain, family members insisted on additional assessments because they equate more extensive assessment with greater caring. A few instances were also noted where even if a nurse was uncertain about a patient's pain, family members insisted that nurses conduct only a brief assessment to avoid bothering the patient. In addition, after self-reports of pain some patients were observed to be uncooperative with additional assessment attempts.

One of the assumptions when this study was designed was that the scope of pain assessment would be determined by the nurse participants, so therefore it would be a valid variable to mediate the relationship between pre-assessment certainty and patient outcome. Observation during the study suggests that this assumption might have been false and therefore the findings need to be interpreted with caution. It appears that pre and post assessment certainty may have been the only variables that some of the nurses had control over. Therefore, it is reasonable to assume that the scope of assessment in this study was contaminated by external factors and does not mediate the relationship between pre-assessment certainty and outcomes. Therefore, a lack of autonomy in choosing assessment scope could be another unexpected uncontrolled variable. A future study could compare nurses in private and public nursing homes in Jordan to explore the variable of autonomy on the assessment choice in regards to family and administrator influence.

The many patients who had diagnoses of mental illness reported in their medical charts might be a possible reason for the very high percentages of variance accounted for by a limited number of variables in this study. There were no exclusion criteria for participants in this study. In retrospect, mental illness, such as depression or schizophrenia, should have been an exclusion 
criterion because it elicits agitated behavior of the participants, which is also a consequence of dementia. Being observed eight times by a stranger (the researcher) has the potential to worsen agitation in those PWD who also had untreated mental illness. When the researcher measured the comorbid burden of the PWD in the study it was discovered that a number of them had mental illness. If the agitation observed in patients after pain treatment was caused by mental illness it would be impossible to accurately measure the outcome of pain treatment in this study. There may be a higher percentage of PWD who have untreated mental illness in nursing homes in the Middle East, including Jordan, than in Western countries because there is more stigma and fewer resources for mental illness; therefore, it would be hard to generalize the results of this study. Consequently, comorbidities, including mental illness, could be another unexpected uncontrolled variable. Future research should control for this variable in order to obtain a more reliable measure of the outcomes of pain treatment in order to explore the relationship between nurses' certainty, the complexity of pain assessment and patient outcomes.

The observers of the patients in this study were both male and the majority of the patients were female. Being observed eight times by a male researcher who is also a stranger may itself have elicited agitation in women with dementia who were socialized to avoid the gaze of men, especially those women who were Muslim. In retrospect, the gender of the observer should have been considered in the research design. If the agitation observed in patients after pain treatment was caused by a socially constructed reaction to a male observer, it would be impossible to accurately measure the outcome of pain treatment in this study. Therefore, the gender of the observer was another unexpected uncontrolled variable in this study. Future research should control for gender differences in order to obtain a more reliable measure of the outcomes of pain 
treatment in order to explore the relationship between nurses' certainty, the complexity of pain assessment and patient outcomes.

This study found that for nurses with high initial levels of certainty, the post assessment level of certainty mediates the relationship between the assessment scope and patient outcomes. Patients of nurses with a high initial level of certainty and high levels of post assessment certainty--regardless of the scope of pain assessment--had better outcomes than patients of nurses with a lower level of post assessment certainty. This finding is consistent with the certainty trajectory in the RCP model. This finding agrees in part with the finding from a previous study by Herr et al. (2011) that nurses who conducted pain assessment in PWD without validating their certainty about the presence of pain did not significantly decrease the level of discomfort of PWD. These corroborating findings suggest the importance of post assessment certainty in the trajectory of decision-making regarding pain treatment in PWD.

This study found that for nurses with low initial levels of certainty, assessment scope and post-assessment certainty are significant predictors of residents' outcomes. There is no previous study examining these variables; however, this finding is consistent with the uncertainty trajectory in the RCP model.

\section{Limitations}

The very high percentages of variance accounted for by a limited number of variables in this study, and the overwhelmingly high support for all hypotheses consistent with the RCP model, raise questions about possible conceptual or methodological limitations in this study.

\section{Conceptual limitations}

While methodological limitations may influence the interpretation of the findings of the study, conceptual limitations may influence the attitude and behavior of the PWD participants. 
The RCP model does not take into account the possible cultural differences in the concept of certainty. It assumes that everyone conceptualizes certainty in the same way. If Jordanian nurses conceptualize certainty differently, the results of this study cannot be generalized crossculturally. One explanation for the very high percentages of variance accounted for by a limited number of variables in this study may be that the concept of certainty itself is culture-bound. If certainty is not culture-bound, what might be culture-bound is the behavior that results form certainty. Nurses in this study may have been more comfortable reporting uncertainty, even if they felt certainty. Self-doubt may have come into play, especially in a culture where women are socialized to be less assertive than women in Western cultures. A future qualitative study to explore the intercultural conceptualizations of certainty is needed.

\section{Methodological limitation}

This study has limitations associated with the study design, the sampling method, and the measurements. The descriptive, correlational design does not permit the inference of causality. Also, the non-probability convenience sampling method of this study may introduce selection bias to the internal validity of the study. The use of the self-report method to collect data may increase the possibility of recall bias, which could affect the reliability of the data and which may influence the results. In addition, since several PWD are cared for by the same nurse, it may be difficult for nurses to recall clearly all needed information about each resident.

When comparing the finding in this study with other studies conducted in the United States, it is important to note that the patient/nurse ratio in Jordan is far higher than in the United States. Although the nurses were instructed to fill out their responses throughout their shift, due to the unusually heavy workload that nurses experience in Jordan, they may have been so busy 
that they left this task to the end of their shift when they were fatigued. This work scenario could have introduced inaccuracies.

Measures of certainty and resident outcomes were clustered within nurses and this clustering was not accounted for in the analyses. It is unknown how this clustering may have influenced the research results. Future research with bigger sample sizes that can account for the possible nesting of findings within nurses or units of nursing homes needs to be conducted.

The nursing education in Jordan is significantly different from the education in the U.S. in terms of exposure to research. In other words, a nurse educated in Jordan may never have had experience in college with self-reporting measurement instruments. Even though the Jordanian nurses were trained on how to use the instruments prior to the study, their basic unfamiliarity with Likert scales could be another limitation to the generalizability of the study findings.

Design contamination may be an additional methodological limitation of this study that likely influenced the findings. Although the researcher made appropriate efforts to keep nurse participants blinded, the nursing home administrators may have unintentionally disclosed the premise of the study. Because there has never been nursing research conducted in Jordan before this study, this researcher had to explain the intention of this project at length in order to gain access to conduct the study. While this researcher requested that the administrators keep the nurse blinded to the premise of the study, this researcher questions whether the administrators followed through on that request. After reflecting on how unusually consistent the data are in supporting the model, this researcher wonders whether the nurse participants were providing the responses that the researcher anticipated rather than honest responses. In retrospect, this researcher reflected that the general atmosphere of work in nursing homes in Jordan is so friendly that there are permeable barriers between nurses and administrators. During the course 
of the study this researcher observed the norm of nurses having frequent friendly chats with administrators in their offices. As all five administrators and the nurse participants were unfamiliar with the nature of nursing research, they may have consulted with each other.

Taking care of elders in the home has been a cultural value in Jordan for many generations. However, nursing homes are gaining prominence in Jordan as life expectancy has increased dramatically and more women are employed outside the home. As a result of increased need, nursing homes have strived to build their reputations. The $\mathrm{NH}$ administrators contacted to participate in this study expressed the hope that that by having research conducted in their facility they would appear superior to their competitors and qualify for more funding opportunities offered by the Ministry of Social Welfare in Jordan. In an effort to encourage the researcher to return and conduct additional studies in their facilities, administrators may have mistakenly tried to get the nurses to respond in a way that supported the researcher's hypotheses.

In addition to subtle pressure from their administrators, the novelty of research initiatives in nursing home settings in Jordan might have influenced the way that nurse participants engaged in the study. Nurse participants may value the research initiative and mistakenly think they are helping the researcher by providing the "right" answer. If particular trajectories of pain assessment and treatment are elicited only in a research context, then the results cannot be generalized to more natural settings. Once nurses in Jordan become more familiar with the scientific approach to nursing research, the same results may fail to appear.

This study may also have suffered from the "Hawthorne Effect." The nurses in this study were aware they were participating in a study and changed their behavior. Because they knew their performance was being measured in some way, they may have given inaccurate responses about their certainty of pain. 


\section{Implications}

If findings of this study can be replicated, results could inform the development of a standard guideline of pain care for PWD, contribute to the development of an intervention, contribute to the design of an evidence-based nursing curriculum in Jordan, and initiate changes in practice or policy related to the assessment and treatment of pain in PWD in Jordan. The findings of this study could yield recommendations to be used by the Ministry of Health in Jordan to redesign the health care delivery and assessment protocol, while also taking into consideration the factors that influence levels of pain and agitation in $\mathrm{NH}$ residents with dementia in Jordan.

Future research should examine the influence of nurses' clinical experience and level of education on their certainty regarding suspected pain in PWD. There is a need for further research to understand the role of the comorbid burden in PWD on the level of nurse' certainty regarding suspected pain and the type of assessment provided to PWD. More translational research is needed to examine structured approaches to widespread application of the RCP model in decision assessment tools for improving the skills of nurses in Jordan caring for NH residents with dementia.

\section{Conclusion}

The RCP model was overwhelmingly supported by findings from this study and may enhance nurses' decision-making and ensure prompt treatment of pain. While findings must be replicated with other samples, this study may provide a new understanding of the relationship between nurses' certainty, assessment scope, and patient outcomes for PWD. 


\section{Study Report: The Association of Comorbid Burden to Patients Outcomes of Nursing \\ Home Residents with Dementia in Jordan}

\section{Introduction to the Problem}

Medical and psychiatric comorbidity is a significant problem for many older patients (Black et al., 2006). For example, $10 \%$ of the older adult population has more than one of the following problems: arthritis, hypertension, heart disease, cerebrovascular diseases, and diabetes (Gloth, 2000). The rates of reported depression among community dwelling older population and nursing home residents ranged from $13.5 \%$ to $14.4 \%$ (Shega et al., 2007). A recent study conducted by Hung, Liu, and Boockvar (2010) on 76 nursing home residents found that $38 \%$ had depression, $25 \%$ had congestive heart failure, $27 \%$ had chronic obstructive lung, and $15 \%$ had stroke. No study has been conducted regarding the comorbid burden among NH residents with dementia in Jordan.

Dementia is a very prevalent problem among the older adults. It is projected that by 2050 , 115.4 million older adults will be affected by dementia worldwide (LoGiudice \&Watson, 2014; Sternberg et al., 2014). As dementia commonly occurs later in life, PWD may also suffer from other medical and psychiatric problems associated with aging. (Black et al., 2006). Compared to community dwelling older adults, nursing home residents with dementia are prone to frailty and at greater risk for increased comorbid burden (Shega, Hougham, Stocking, Cox-Hayley, \& Sachs, 2005). However, there is limited information about the consequences of the comorbid burden among nursing home residents with dementia. The comorbid burden in PWD is often associated with serious adverse health outcomes, such as functional disability, institutionalization, and death. Death among NH residents with dementia may be caused by comorbidities involving any of the following conditions: malnutrition, urinary incontinence, 
pressure ulcers, delirium, cardiovascular diseases, depression, chronic obstructive pulmonary disease, diabetes mellitus, cerebrovascular disease, or respiratory disease (Black et al.).

According to Hung, Liu, and Boockvar (2010), some medical conditions, such as cardiovascular disease, could be a risk factor for dementia. Conversely, dementia negatively impacts many aspects of the individuals' daily life, such as the ability to adhere to lifestyle recommendations, which may put the PWD at a greater risk for medical and psychiatric conditions. Conditions such as degenerative joint disease, fractures, pressure ulcers, post-stroke syndrome, depression and anxiety, and cancer could lead to pain and agitation, which are typical problems in PWD (Hung et al.). Nurses need to be aware of and understand the impact that comorbidities have on patient outcomes in order to effectively assess and treat pain and agitation in PWD.

Many studies have shown that PWD have difficulty communicating with nurses and may be unable to verbally self-report symptoms due to cognitive impairment and aphasia (Kovach, 2013; Rudich, Lerman, Gurevich, \& Shahar, 2010; Wilson, Uhelski, \& Fuchs, 2008). However, relationships between dementia, the ability to verbally self-report symptoms, and comorbid burden are very complex and it is not clearly known how these variables contribute to patient outcomes of PWD. It is not clearly known whether PWD who have comorbidities experience worse outcomes because of their inability to verbally self-report symptoms or because of comorbid burden itself.

It has been reported that agitated behaviors can interfere with the accurate diagnosis and effective treatment of comorbidities (Snow et al., 2005). In addition, it is known that the inability to verbally self-report symptoms in PWD may also complicate the accurate diagnosis of comorbidities (Black et al., 2006). However, it is not clearly known if comorbid burden can 
predict the outcomes of pain and agitation in PWD. Further, it is not known if the inability to verbally self-report symptoms predicts worse health outcomes in PWD regardless of the degree of comorbid burden in PWD.

Therefore, this study first describes the comorbid burden of people with dementia in Jordanian nursing homes and their ability to verbally report symptom, then examines whether comorbid burden predicts pain and agitation in PWD, and also examines whether the ability of PWD to verbally self-report symptoms mediates the relationship between comorbid burden and patient outcomes.

We hypothesized that severity of dementia and comorbid burden are predictors of the level of pain and agitation in PWD. We also hypothesized that the relationship between comorbid burden and patient outcomes of pain and agitation in PWD could be explained by their ability to verbally self-report symptoms (Table A). We expected that NH residents with greater comorbid burden and more severe dementia would be more likely to have high levels of pain and agitation. The identification of comorbid burden associated with pain and agitation can alert nurses to the potential need for pain management in PWD.

Table A: Research Questions and Hypothesis.

\section{Research Questions}

1. What is the comorbid burden of nursing home residents with dementia in Jordan?

2. What is the ability of people with dementia residing in Jordanian nursing homes to verbally report symptoms?

\section{Research Hypotheses}

1. Controlling for age, do severity of dementia and comorbid burden predict patient outcomes of pain and agitation in PWD?

2. Controlling for age and severity of dementia, does ability to verbally self-report symptoms mediate the relationship between comorbid burden and patient outcomes of pain and agitation in PWD? 


\section{Method}

\section{Data Analysis}

The SPSS 20 for Windows (SPSS Inc., Chicago, IL, USA) was used for the statistical analyses. The assumptions of normal distribution, homogeneity of variances, linearity and independence of data were tested and outliers were excluded. Descriptive statistics were used to describe comorbid burden, ability to verbally self-report symptoms, and demographic data. A hierarchical regression method was used to examine the significant predictors of residents' outcomes and mediating effects of variables. Tolerance values of less than 0.1 for independent variables indicated that multicollinearity was not present. In hierarchical multiple regression equations the order of entry of the variables is important. The order of entry the variables was based on their clinical importance as well as the interest of the researcher. Ability to verbally self-report symptoms is the main dependent variable and was entered into the regression models in the final step. The order of entry into the model yielded the variability in the levels of pain and agitation that was uniquely accounted for by the ability to verbally self-report symptoms.

\section{Results}

\section{Description of Participants}

The mean age for the 76 residents was 72.52 years (SD 9.45, range 55-95) and 56.0\% were men. The average length of stay for the residents was 41.7 months (SD 10.33, range 3593). Twenty-two percent $(n=17)$ of the residents had severe dementia; the mean MMSE score was 14.44 (SD 6.54, range 0-23).

\section{Description of Comorbid Conditions and Ability to Verbally Report Symptoms}

Thirty seven percent of the 76 residents had Hypertension, $14.4 \%$ had visual impairment, $32.8 \%$ had musculoskeletal disorders, $30.5 \%$ had diabetes mellitus, $16.4 \%$ had and 
gastro $\square$ intestinal disorders, $9.2 \%$ had chronic heart failure $25 \%$ had chronic obstructive lung disease, $13 \%$ had genitourinary problems, and $17 \%$ had stroke, $11 \%$ had cancer, $10 \%$ had depression, and $11 \%$ had schizophrenia. Twenty one percent of the residents suffered from comorbid problems that are considered severe based on the comorbid burden scale. Twenty-two percent $(n=17)$ of the residents had severe dementia; the mean MMSE score was 14.44 (SD 6.54 , range $0-23$ ). Thirty six percent of the 76 residents with dementia were able to verbally selfreport symptoms $(\mathrm{n}=26)$ and $64 \%$ were unable to self-report $(\mathrm{n}=50)$.

\section{Hypothesis One}

Tables 13-15 describe the relative contribution of severity of dementia and comorbid burden as predictors of agitation and pain outcomes. Hypothesis one was supported. As seen in Tables 13-15, after controlling for age, at Step 2 severity of dementia significantly predicted agitation and pain 1 and pain 2 scores. The percentage of variance accounted for was $10.9 \%$ for agitation, $6.3 \%$ for pain 1 and $9.5 \%$ for pain 2 . At Step 3 comorbid burden uniquely predicted a statistically significant percentage of variance in all outcomes ( $13.1 \%$ for agitation, $11.2 \%$ for pain 1 and $12.7 \%$ for pain 2). Overall age, severity of dementia, and comorbid burden predicted $32.3 \%$ of the variance in agitation, $22.9 \%$ of the variance in pain 1 , and $30.7 \%$ of the variance in pain2.

Table 13. Summary of Hierarchical Regression Analysis with Agitation Scores as Criterion $(\mathbf{N}=76)$.

\begin{tabular}{lccccc}
\hline Step and Predictor Variable & $\mathrm{R}^{2}$ & $\Delta \mathrm{R}^{2}$ & $\beta$ & $\mathrm{t}$ & $\mathrm{p}$ \\
\hline Step 1: & & & & & \\
Age & .083 & $.083^{*}$ & .387 & 3.66 & $<.001^{*}$ \\
Step 2: & & & & & \\
Age & & & .099 & .807 & .422 \\
Severity of Dementia & .192 & $.109^{*}$ & -.487 & -3.88 & $<.001^{*}$ \\
Step 3: & & & & & \\
Age & & & .149 & 1.32 & .191 \\
Severity of Dementia & & & -.390 & -3.42 & $.001^{*}$ \\
Comorbid Burden & .323 & $.131^{*}$ & .359 & 3.97 & $<.001^{*}$ \\
\hline
\end{tabular}




\begin{tabular}{lccccc}
\hline$F_{(3,72)}=17.55, p<.0001$ & & & & \\
\hline & $* 0.05$ \\
Table 14. Summary of Hierarchical Regression Analysis with Pain \\
= 76).
\end{tabular}

Table 15. Summary of Hierarchical Regression Analysis with Pain 2 Scores as Criterion (N =76).

\begin{tabular}{lccccc}
\hline Step and Predictor Variable & $\mathrm{R}^{2}$ & $\Delta \mathrm{R}^{2}$ & $\beta$ & $\mathrm{t}$ & $\mathrm{p}$ \\
\hline Step 1: & & & & & \\
Age & .085 & $.085^{*}$ & .335 & 3.10 & $.003^{*}$ \\
Step 2: & & & & & \\
Age & & & .050 & .349 & .691 \\
Severity of Dementia & .180 & $.095^{*}$ & -.418 & -3.669 & $<.001^{*}$ \\
Step 3: & & & & & \\
Age & & & .102 & .878 & .383 \\
Severity of Dementia & & & -.331 & -3.21 & $.005^{*}$ \\
Comorbid Burden & .307 & $.127^{*}$ & .370 & 3.49 & $<.001^{*}$ \\
$F_{(3,72)}=15.27, p<.0001$ & & & & & \\
\hline$* p<.05$ & & & & &
\end{tabular}

\section{Hypothesis Two}

The notion that ability to verbally report symptoms mediates the relationship between comorbid burden and patient outcomes of pain and agitation was not supported. As seen in the betas in Step 3 of Tables $16-18$, when ability to verbally report symptoms was added to the models, the betas for comorbid burden remained statistically significant. However, the ability to verbally report symptoms uniquely accounted for a significant portion of the variance in each outcome $(15.3 \%$ of the variance in agitation, $20.1 \%$ of the variance in pain 1 , and $14.1 \%$ of the 
variance in pain2). Overall age, severity of dementia, comorbid burden and ability to verbally report symptoms predicted $47.6 \%$ of the variance in agitation, $43.0 \%$ of the variance in pain 1 , and $44.8 \%$ of the variance in pain 2 .

Table 16. Summary of Hierarchical Regression Analysis with Agitation Scores as Criterion $(\mathbf{N}=\mathbf{7 6})$.

\begin{tabular}{lccccc}
\hline Step and Predictor Variable & $\mathrm{R}^{2}$ & $\Delta \mathrm{R}^{2}$ & $\beta$ & $\mathrm{t}$ & $\mathrm{p}$ \\
\hline Step 1: & & & & & \\
Age & & & .099 & .807 & .422 \\
Severity of Dementia & .192 & $.192^{*}$ & -.487 & -3.88 & $<.001^{*}$ \\
Step 2: & & & & & \\
Age & & & .149 & 1.32 & .191 \\
Severity of Dementia & .323 & $.131^{*}$ &. .390 & -3.42 & $.001^{*}$ \\
Comorbid Burden & & & & 3.97 & $<.001^{*}$ \\
Step 3: & & & .062 & .632 & .529 \\
Age & & & -.035 & -.297 & .767 \\
Severity of Dementia & & & .315 & 3.19 & $.009^{*}$ \\
Comorbid Burden & .476 & $.153^{*}$ & -.584 & -5.33 & $<.001^{*}$ \\
Ability to Verbally Self-Report & & & & & \\
$F(4,71)=25.15, p<.0001$ & & & & & \\
\hline$* p<.05$ & & & & & \\
Table 17. Summary & & & & & \\
\hline
\end{tabular}

Table 17. Summary of Hierarchical Regression Analysis with Pain 1 Scores as Criterion (N = 76).

\begin{tabular}{lccccc}
\hline Step and Predictor Variable & $\mathrm{R}^{2}$ & $\Delta \mathrm{R}^{2}$ & $\beta$ & $\mathrm{t}$ & $\mathrm{p}$ \\
\hline Step 1: & & & & & \\
Age & & & .009 & .067 & .947 \\
Severity of Dementia & .117 & $.117^{*}$ & -.389 & -3.36 & $.001^{*}$ \\
Step 2: & & & & & \\
Age & & & .052 & .442 & .659 \\
Severity of Dementia & .229 & $.112^{*}$ &. .337 & -2.86 & $.006^{*}$ \\
Comorbid Burden & & & & & \\
Step 3: & & & .026 & .249 & $<.001^{*}$ \\
Age & & & -.019 & -.146 & .804 \\
Severity of Dementia & & & .346 & 4.19 & $.010^{*}$ \\
Comorbid Burden & .430 & $.201^{*}$ & -.524 & -4.41 & $<.001^{*}$ \\
Ability to Verbally Self-Report & & & & & \\
$F(4,71)=18.48, p<.0001$ & & & & & \\
\hline$* p<.05$ & & & & &
\end{tabular}

Table 18. Summary of Hierarchical Regression Analysis with Pain 2 Scores as Criterion (N $=76)$.

\begin{tabular}{lccccc}
\hline Step and Predictor Variable & $\mathrm{R}^{2}$ & $\Delta \mathrm{R}^{2}$ & $\beta$ & $\mathrm{t}$ & $\mathrm{p}$ \\
\hline Step 1: & & & & & \\
Age & & & .050 & .349 & .691 \\
Severity of Dementia & .180 & $.180^{*}$ & -.418 & -3.669 & $<.001^{*}$
\end{tabular}




\begin{tabular}{|c|c|c|c|c|c|}
\hline \multicolumn{6}{|l|}{ Step 2: } \\
\hline Age & & & .102 & .878 & 383 \\
\hline Severity of Dementia & & & -.331 & -3.21 & $.006^{*}$ \\
\hline $\begin{array}{l}\text { Comorbid Burden } \\
\text { Step 3: }\end{array}$ & .307 & $.127^{*}$ & .370 & 3.49 & $<.001 *$ \\
\hline Age & & & .023 & .224 & .823 \\
\hline Severity of Dementia & & & -.062 & -.498 & 626 \\
\hline Comorbid Burden & & & 290 & 3.56 & $.008^{*}$ \\
\hline $\begin{array}{l}\text { Ability to Verbally Self-Report } \\
F_{(4,71)}=19.34, p<.0001\end{array}$ & .448 & $.141^{*}$ & -.529 & -4.51 & $<.001 *$ \\
\hline
\end{tabular}

\section{Discussion}

This study showed a high prevalence of medical and psychiatric comorbidity among PWD residing in Jordanian nursing homes. The most prevalent comorbidities in this study were cardiovascular disorders, diabetes mellitus, gastrointestinal disorders, chronic obstructive lung diseases, musculoskeletal disorder, and cancers. Most residents had at least four medical conditions at the time of the study. This finding is consistent with previous studies that have shown the high prevalence of comorbidities among NH residents (Bell et al., 2015: Black et al., 2006; Hung et al., 2010).

The finding that the majority of residents in this study were unable to verbally self-report symptoms is consistent with other studies. For example, when Fox et al. (2000) reviewed the literature on assessment and treatment of pain in American nursing homes, they found that pain is still under-reported by the majority of PWD. Many previous studies have reported that PWD experience pain differently than cognitively intact older adults (Kovach, 2013; Rudich, Lerman, Gurevich, \& Shahar, 2010; Wilson, Uhelski, \& Fuchs, 2008). For example, a recent study found that as the severity of dementia advanced, the affective experience of pain becomes more distorted (Wilson et al., 2008). This distortion interferes with the ability of PWD to verbally selfreport pain (Kovach, 2013), suggesting that interventions designed to encourage PWD to 
verbally self-report pain could result in better patients outcomes.

Results from this study support the hypothesis that comorbid burden and severity of dementia are significant predictors of patient outcomes, a finding consistent with previous studies examining these variables and finding them critical factors in determining the outcomes of nursing home residents (McLachlan et al., 2011). Monroe et al. (2012) found that severity of dementia was negatively associated with the level of pain in PWD. In addition, differences in experience of pain were due to variance in severity of dementia. Similarly a recent study by Sternberg, Bentur, and Shuldiner (2014) found that NH residents with dementia had poor health outcomes due to their cognitive impairment. It is understood that cognitive impairment distorts the experience of pain and limits the ability of PWD to understand treatment options and articulate their emotional needs.

Some painful medical comorbidities, such as cancer and dementia, may lead to severe unrelieved pain. Unrelieved pain is also associated with psychiatric comorbidities, such as depression, which may worsen patient outcomes of pain and agitation (Monroe, Carter, Feldt, Tolley, \& Cowan, 2012). According to Damush, et al. (2016), teaching patients how to cope with and manage the symptoms of their medical and psychiatric comorbidities can improve painrelated outcomes. Similarly, LoGiudice and Watson (2014) found that effective management of psychiatric and medical comorbidities is an essential component of a better quality of life (Damush, et al.). Consistent with these findings, another study examining how to optimize patient outcomes of PWD, found that managing psychiatric conditions, such as depression, would improve analgesia (Shega et al., 2007). Indeed, lower comorbid burden was one of the significant predictors of better patient outcomes and quality of end life in PWD (Sternberg et al., 2014). 
This study found that the relationship between comorbid burden and patient outcomes could not be explained by ability to verbally self-report symptoms. This means that variations in both comorbid burden and ability to verbally self-report symptoms significantly account for variations in the level of pain and agitation in PWD. However, variations in comorbid burden insignificantly account for variations in the ability of PWD to verbally self-report symptoms. The finding regarding the insignificant mediation effect of ability to verbally self-report symptoms is consistent with two previous studies: one found medical and psychiatric comorbidities themselves can cause pain and agitation (Black et al. (2006); the other study found that comorbidity was not a significant predictor of ability to verbally self-report pain in PWD (Cohen-Mansfield and Lipson, 2003).

PWD who had greater comorbid burden had worse outcomes of pain agitation and PWD who had less comorbid burden had better outcomes of pain, although not necessarily because of ability to verbally self-report pain. This finding is consistent with a previous study that found the unrelieved pain in PWD who had multiple chronic conditions could not be merely explained by their communication problems (Black et al., 2006). Further, it was found that medical comorbidity could complicate the accurate assessment and effective treatment of pain in PWD (Black et al.). This finding suggests that nurses should be particularly alert in assessing pain in PWD who have multiple medical and psychiatric conditions. Nursing home residents with dementia with a greater comorbid burden are at risk for inadequate medication reconciliation. The interruption of analgesics and anti-psychotic medication can lead to withdrawal symptoms including discomfort, restlessness, anxiety, and agitation (Hung, Liu, \& Boockvar, 2010). Health care providers are cautious about overmedicating frail and cognitively impaired PWD because some analgesics may cause life-threatening adverse effects due to drug-drug and disease-drug 
interactions (McLachlan et al., 2011). Often PWD tend to receive less analgesia and are more likely to have worse outcomes of pain and agitation.

\section{Limitations}

This study has several limitations related to the study sample, the sampling method, design, and measurement. First, This study was conducted in one geographical area, which may limit the generalizability of study findings. Second, this study was conducted in nursing homes, therefore the findings of this study may not be applicable to community-dwelling PWD. Further, the descriptive, correlational design does not permit the inference of causality. Also, the nonprobability convenience sampling method of this study may introduce selection bias to the internal validity of the study. The fact that two raters collected the data of the study could results in measurement errors, which could be an additional threat to the internal validity of the study. It has been reported that agitation could interfere with the accurate diagnosis of comorbidity. Therefore, the sample may have had more comorbid conditions than had been diagnosed and measured for this analysis.

\section{Implications}

The findings of this study could contribute to the development of an intervention study where nurses receive educational sessions about comorbid burden in PWD. Another intervention study where nurses receive educational sessions on how they encourage PWD to verbally and meaningfully self-report symptoms could be developed. Also the findings of this study could contribute to the redesign of nursing curriculum related to the assessment and treatment of pain, while also taking into consideration the cultural and environmental factors that influence experience of pain and agitation in PWD in Jordan. 


\section{Future recommendation for Research}

Future research should examine the influence of residents' length of stay, severity of dementia, and level of patient education and self-management on their comorbid burden scores. A Future intervention study on dealing with greater assessment of non-verbal PWD should be conducted. Future research with bigger sample sizes that can account for the possible nesting of findings within units of nursing homes needs to be conducted. Replication studies in other Middle Eastern countries are needed to validate the findings of this study.

\section{Conclusion}

Comorbid burden is a significant problem among NH residents with dementia and significantly predicts patient outcomes of pain and agitation. PWD often have difficulty selfreporting their symptoms to nurses and the ability to verbally self-report symptoms significantly predicts patient outcomes. Given the projected increase of the percentage of PWD in the next few decades, improved understanding of comorbid burden and the ability of PWD to verbally self-report symptoms and how they relate to the problem of unrelieved pain in PWD is crucial.

\section{Chapter Summary}

This chapter reported the design, methods, setting and participants of the study. The results of the nurses' certainty about pain in PWD, type of pain assessment, severity of dementia, comorbid burden, communication deficit, and patient outcomes were reported and the findings of the associations of these variables were discussed. Limitations, implications, and recommendations for future research were presented. The findings of this descriptive correlation study filled gaps in knowledge and would contribute to nursing science by highlighting some potential limitations as well as suggesting future replicating studies before being applied to practice. 


\section{CHAPTER 5}

This chapter presents a summary of the major findings of this study. Proposed implications for nursing education, nursing clinical practice, and public policy are offered. Study limitations and threats to the validity are reviewed. Recommendations for future research will be explained. This study found an excessively high percentage of variances accounted for by nurses' level of certainty due to potential measurement errors, the preliminary nature of this study, and potential unexpected uncontrolled confounding variables. Therefore, the discussion of results and the interpretation of findings should be presented with caution and replicated before being applied to practice or theory derivation.

\section{Synthesis of Findings}

Unrelieved pain in PWD is a significant problem. Pain in PWD is still under-assessed and undertreated. Findings from this study indicate that pre-assessment level of nurses' certainty, assessment scope, and post assessment level of nurses' certainty are significant predictors of patient outcomes of pain and agitation in PWD in Jordan. These findings are inconsistent with literature that shows that agitation is caused by multiple factors. Nurses' internal beliefs about a person's pain level would not logically predict this high of a percentage of variance. The way that nurses responded to Likert scale of certainty regarding suspected pain in PWD in the study was highly supportive to the researcher's hypotheses due to many reasons. First, Recall bias of nurses due to their unusually heavy workload as well as nurses' unfamiliarity with Likert scales could introduce inaccuracies. Second, the possible disclosure of the study premise by $\mathrm{NH}$ administrators as well as their subtle pressure on nurses and the novelty of research initiatives in nursing home settings in Jordan might have influenced the way that nurse participants engaged in the study. However, consistent with other literature, this study shows that nurses caring for PWD 
are still uncertain regarding suspected pain, which may contribute to poor outcomes of pain and agitation. Furthermore, this study found that nurses are not well educated on how they should conduct the assessment of pain in PWD. In this study, nurses underestimated the reliability of patients' signs and symptoms and their self-reports to predict patient outcomes. Instead, nurses did believe their internal feelings about a person's pain level and then they did objective assessment of pain and did believe the subjective nature of a patient's self-report. The nurses in this study turned the hierarchy of pain assessment upside down, which may explain the poor patient outcomes and lack of certainty.

Because of the very high percentages of variance accounted for by a limited number of variables in this study, the researcher examined other variables including comorbid burden and ability to verbally self-report to see whether they predicts the outcomes of pain and agitation in PWD. Findings from this study indicate that comorbid burden and ability to verbally self-report symptoms are significant predictors of patient outcomes of pain and agitation in PWD in Jordan. These findings are consistent with previous studies examining these variables and finding them critical factors in determining the outcomes of nursing home residents. These findings suggest that nurses should be particularly alert in assessing pain in PWD who have multiple medical and psychiatric conditions and/or are unable to verbally self-report symptoms. Also, these findings suggest that interventions designed to encourage PWD to verbally self-report pain could result in better patients outcomes.

\section{Potential Implications}

If findings of this study can be replicated, results could inform the development of a standard guideline of pain care for PWD, contribute to the development of an intervention, contribute to the design of an evidence-based nursing curriculum in Jordan, and initiate changes 
in practice or policy related to the assessment and treatment of pain in PWD in Jordan. The findings of this study could yield recommendations to be used by the Ministry of Health in Jordan to redesign the health care delivery and assessment protocol, while also taking into consideration the factors that influence levels of pain and agitation in $\mathrm{NH}$ residents with dementia in Jordan.

\section{Nursing Education}

Despite the significance of unmet comfort needs of NH residents, nursing schools in Middle Eastern countries, including Jordan, lag behind Western countries when it comes to the assessment and treatment of pain. Nursing schools in Jordan do not have gerontology departments where faculty are conducting and disseminating gerontological research and writing nursing curriculum. In fact, there are no faculty members in nursing schools in Jordan with a degree or certificate in the field of gerontology. Nursing coursework in Jordan is focused on pediatric and adult health but does not cover gerontological issues like dementia care, palliative care, or long-term care. In fact, the nursing homes in Jordan are not considered potential clinical sites for nursing students. Students are unprepared by their nursing education to assess and manage pain in PWD. Progressive nursing school deans in Jordan are beginning to develop a vision for supporting new faculty in pursuing academic research careers in gerontology.

According to Eid, Manias, Bucknall, and Almazrooa (2014), Jordanian nurses still have misconceptions, knowledge deficits, and wrong beliefs about pain assessment and management. Myths about the potential for PWD to become addicted to pain medicine persist among nurses who have insufficient information about the phenomenon of pain and the process of pain management for PWD who have a different presentation of pain. If nursing graduates lack knowledge about and clinical experience with PWD, they may feel uncertainty about pain 
assessment and delay treatment of pain when employed as nurses. The findings of this study could contribute to the development of evidence-based nursing curriculum in Jordan and other Middle Eastern countries. Specialized courses in gerontology could include a unit about the assessment and treatment of pain in PWD. Simulations could be developed that allow nursing students to experience pain treatment trajectories. The RCP model could serve as a framework for these experiential learning scenarios. Students could reflect on their own critical thinking and decision-making processes and also observe those trajectories unfold as their fellow students engage in simulation.

Nursing students exposed to curriculum rich in gerontological research may pursue graduate studies in this area. If enough Jordanian nurses pursue advanced degrees in gerontology, universities might be willing to hire faculty to develop departments of geriatric health nursing. Jordan has a history of supporting other nursing research initiatives in their universities such smoking cessation, diabetes prevention, and birth control. Because Jordanian culture values elders it seems reasonable to assume that there could be funding potential from the government for gerontological nursing research centers. Emerging programs of research will empower nurses with the professional education necessary to overcome barriers for quality pain care in nursing homes (Eid et al., 2014).

Exposing nursing student to research about nursing that is generated by nurses for nurses helps students begin to see the field of nursing as autonomous rather than dependent on medicine. This empowering experience is especially important for nursing students in Jordan and other Middle Eastern countries because nursing is an emerging discipline there.

\section{Clinical Practice in Nursing Home}

This study adds to the gathering evidence about nurses' critical thinking and decision- 
making processes, which guide their practice. Implications for practice in nursing homes are many. The findings of this study could aid administrators in developing orientation and ongoing training for their nurse employees on managing pain for PWD. Administrators could use the RCP model as a framework for discussing uncertainty about pain in PWD. It could provide a common language for nurse mangers and nurses to evaluate and improve nursing practice. Used as a tool for performance reviews nurse could demonstrate their growing self-confidence in their assessment and treatment of pain in PWD. By incorporating a standard pain management guideline, nurse may become more confidently and consistently administer analgesic to those PWD who are in pain (Gallagher \& Long, 2011). Increase job satisfaction could result from nurses becoming more aware of the value of their knowledge and experience and in delivering prompt pain relief in PWD. Increase job satisfaction and self-confidence of nurses could decrease nurses' burnout rate.

The findings of this study could be used to guide the development of a standard pain management guideline tailored to the unmet needs of PWD. This guideline could help nurses establish consistency in assessing and managing pain in PWD (Herr, Coyne, McCaffery, Manworren \& Merkel, 2011). Consistency among care providers facilitates communication and evaluation of pain management and clinical decisions (Herr et al., 2011). Consistent and effective treatment of pain helps families and patients develop trusting relationship with nurses. With this trusting relationship, families become satisfied that their loved ones are in good hands. Increase of patients' satisfaction and retention rate protect and defend the reputation of nursing homes and increase the cost-effective rate of pain treatment in PWD.

This guideline could help nurses to effectively assess and promptly treat pain in PWD. Relieved pain in PWD could improve physical function, appetite, social life, sleep and activity 
pattern, mobility, immune function, and quality of life. Improved quality of life of PWD increases their satisfaction. When family members see their loved ones satisfied with their quality of life, they will not be obligated to leave their jobs, which relieve the caregiving related financial burdens.

The RCP model of this study describes possible trajectories of the decision-making process nurses employ to assess and treat pain in nursing home residents with dementia. NH administrators may be able to evaluate nurses' critical thinking and decision-making skills and train those nurses who still are struggling. Findings of a recent study confirmed that improvements in pain management in PWD have been demonstrated following training session and workshops for nurses involved in caring for NH residents (Achterberg et al., 2013).

\section{Policies}

The findings of this study imply that there is a need to improve the quality of pain care delivered for PWD living in nursing homes in Jordan. The findings of this study could be used as a proposal given to the government or policymakers to reform the pain practice in all nursing homes in Jordan. The statistics mentioned in this study about the significant of unrelieved pain in PWD would grab the attention of the government or health policymakers in Jordan. In Jordan, older adult people are about $8 \%$ of the population. Even though the number older adults is going to multiply over the coming ten years, it has been given a little attention by health-policy makers and health researchers (Almomani, McDowd, Bani-Issa,\& Almomani, 2014). Nursing researchers and health policy makers should be afraid of increasing the life expectancy of older adults with parallel increase of their proportion and the progressive decline in their quality of life.

The findings of this study may encourage the government to propose a proportion of its 
budget for funding research and projects in pain and dementia. Inadequate financial support could impede the feasibility and sustainability of these projects (Estes et al., 2013). This study would be a strong message to the government and policymakers that the future policy in Jordan needs to be tailored to address the unmet comfort needs of $\mathrm{NH}$ residents.

\section{Limitations}

The very high percentages of variance accounted for by a limited number of variables in this study, and the overwhelmingly high support for all hypotheses consistent with the RCP model, raise questions about possible conceptual or methodological limitations in this study.

\section{Conceptual Limitations}

While methodological limitations may influence the interpretation of the findings of the study, conceptual limitations may influence the attitude and behavior of the PWD participants. The RCP model does not take into account the possible cultural differences in the concept of certainty. It assumes that everyone conceptualizes certainty in the same way. If Jordanian nurses conceptualize certainty differently, the results of this study cannot be generalized crossculturally. One explanation for the very high percentages of variance accounted for by a limited number of variables in this study may be that the concept of certainty itself is culture-bound. If certainty is not culture-bound, what might be culture-bound is the behavior that results form certainty. Nurses in this study may have been more comfortable reporting uncertainty, even if they felt certainty. Self-doubt may have come into play, especially in a culture where women are socialized to be less assertive than women in Western cultures. A future qualitative study to explore the intercultural conceptualizations of certainty is needed.

\section{Methodological Limitation}

This study has limitations associated with the study sample, design, the sampling method, 
and the measurements. This study was conducted in one geographical area, which may limit the generalizability of study findings. Also, this study was conducted in nursing homes, therefore the findings of this study may not be applicable to community-dwelling PWD. Furthermore, The study sample has NH residents a wide age range (55-90), which is different from most North American or European NH residents. Thus, health outcomes of pain and agitation may be different in this sample than in other studies that typically focus only on NH residents who are 65 years and older.

The descriptive, correlational design does not permit the inference of causality. Also, the non-probability convenience sampling method of this study may introduce selection bias to the internal validity of the study. The use of the self-report method to collect data may increase the possibility of recall bias, which could affect the reliability of the data and which may influence the results. In addition, since several PWD are cared for by the same nurse, it may be difficult for nurses to recall clearly all needed information about each resident. The fact that two raters collected the data of the study could results in measurement errors, which could be an additional threat to the internal validity of the study. It has been reported that agitation could interfere with the accurate diagnosis of comorbidity. Therefore, the sample may have had more comorbid conditions than had been diagnosed and measured for this analysis.

When comparing the finding in this study with other studies conducted in the United States, it is important to note that the patient/nurse ratio in Jordan is far higher than in the United States. Although the nurses were instructed to fill out their responses throughout their shift, due to the unusually heavy workload that nurses experience in Jordan, they may have been so busy that they left this task to the end of their shift when they were fatigued. This work scenario could have introduced inaccuracies. 
Measures of certainty and resident outcomes were clustered within nurses and this clustering was not accounted for in the analyses. It is unknown how this clustering may have influenced the research results. Future research with bigger sample sizes that can account for the possible nesting of findings within nurses or units of nursing homes needs to be conducted.

The nursing education in Jordan is significantly different from the education in the U.S. in terms of exposure to research. In other words, a nurse educated in Jordan may never have had experience in college with self-reporting measurement instruments. Even though the Jordanian nurses were trained on how to use the instruments prior to the study, their basic unfamiliarity with Likert scales could be another limitation to the generalizability of the study findings.

Design contamination may be an additional methodological limitation of this study that likely influenced the findings. Although the researcher made appropriate efforts to keep nurse participants blinded, the nursing home administrators may have unintentionally disclosed the premise of the study. Because there has never been nursing research conducted in Jordan before this study, this researcher had to explain the intention of this project at length in order to gain access to conduct the study. While this researcher requested that the administrators keep the nurse blinded to the premise of the study, this researcher questions whether the administrators followed through on that request. After reflecting on how unusually consistent the data are in supporting the model, this researcher wonders whether the nurse participants were providing the responses that the researcher anticipated rather than honest responses. In retrospect, this researcher reflected that the general atmosphere of work in nursing homes in Jordan is so friendly that there are permeable barriers between nurses and administrators. During the course of the study this researcher observed the norm of nurses having frequent friendly chats with 
administrators in their offices. As all five administrators and the nurse participants were unfamiliar with the nature of nursing research, they may have consulted with each other.

Taking care of elders in the home has been a cultural value in Jordan for many generations. However, nursing homes are gaining prominence in Jordan as life expectancy has increased dramatically and more women are employed outside the home. As a result of increased need, nursing homes have strived to build their reputations. The NH administrators contacted to participate in this study expressed the hope that that by having research conducted in their facility they would appear superior to their competitors and qualify for more funding opportunities offered by the Ministry of Social Welfare in Jordan. In an effort to encourage the researcher to return and conduct additional studies in their facilities, administrators may have mistakenly tried to get the nurses to respond in a way that supported the researcher's hypotheses. In addition to subtle pressure from their administrators, the novelty of research initiatives in nursing home settings in Jordan might have influenced the way that nurse participants engaged in the study. Nurse participants may value the research initiative and mistakenly think they are helping the researcher by providing the "right" answer. If particular trajectories of pain assessment and treatment are elicited only in a research context, then the results cannot be generalized to more natural settings. Once nurses in Jordan become more familiar with the scientific approach to nursing research, the same results may fail to appear.

This study may also have suffered from the "Hawthorne Effect." The nurses in this study were aware they were participating in a study and changed their behavior. Because they knew their performance was being measured in some way, they may have given inaccurate responses about their certainty of pain. 


\section{Recommendations for Future Research}

Future research examining more carefully intercultural conceptualizations of certainty is needed. The concept of certainty itself could be culture bond. In another words, Jordanian nurses in this study might conceptualize certainty differently than Western nurses. A future qualitative study to explore the conceptualization of certainty among Jordanian nurses caring for PWD. In this qualitative study, the research will check if what the nurses put on the Likert scale of certainty match what they really think of certainty regarding suspected pain in PWD.

Because of the excessively high percentage of variances accounted for by nurses' level of certainty due to potential unexpected uncontrolled confounding variables, future research should control for these confounding variables. Future research should control for gender differences and mental conditions in order to obtain a more reliable measure of the outcomes of pain treatment in order to explore the relationship between nurses' certainty, the complexity of pain assessment and patient outcomes. Also, a future study could compare nurses in private and public nursing homes in Jordan to explore the variable of autonomy on the assessment choice in regards to family and administrator influence. Furthermore, a future study could include an evaluation of nurses' knowledge of pain in PWD in order to explore this variable's influence on the mediating effect of assessment scope.

Future research should examine the influence of nurses' clinical experience and level of education on their certainty regarding suspected pain in PWD. There is a need for further research to understand the role of comorbid burden in PWD on the level of nurse' certainty regarding suspected pain and type of assessment provided to PWD. Future research should examine the influence of residents' length of stay, severity of dementia, and level of patient education and self-management on their comorbid burden scores. Future intervention studies on 
dealing with greater assessment of non-verbal PWD should be conducted. More translational research is needed to examine structured approaches to widespread application of the RCP model in decision assessment tools for improving the skills of nurses caring for $\mathrm{NH}$ residents with dementia.

Future research examining the effect of time to administer analgesics and the predictability of outcomes in PWD are needed. Time to administer analgesics is the time that nurses take to respond to behavior changes and suspected pain in residents with dementia and administers analgesics. This study only captured two days of observation for each participant. A study that would entail observing participants for several days may provide more robust findings. Careful consideration would need to be made when designing a study of this nature. Extended observations may produce increased levels of agitation in those $\mathrm{NH}$ residents with mild to moderate dementia who may be aware someone is watching them.

Replication studies with larger sample size and in other Middle Eastern countries are needed to validate the findings of this study. Also the findings of this study could be validated in other clinical setting with different populations who have the same communication or cognitive problem like neonates, children with disabilities, stroke and ICU patients. Specifically examining the effects of nurses' certainty and scope of pain assessment and predictability of patient outcomes are needed. For example, a recent study has reported that pain is a neglected problem in children with disabilities (Ohansson, Carlberg, \& Jylli, 2010; Massaro et al., 2014). Also according to another study, the main barrier to assessment and treatment of pain in those patients is the complexity of pain assessment. Nurses are still unconfident in their decision making process of pain assessment and treatment in children with disabilities (Breau \& Camfield, 2011). 
The findings of this study would guide future experimental research regarding nursing interventions designed to improve the comfort needs of nursing home residents with dementia in Jordan. The factors that are found in my dissertation to be significantly associated with outcomes and_are amenable to change through nursing intervention would be studied in the future. For example, the findings of this study will inform conducting an experimental study to determine the efficacy of using either self-report pain tools or the multidimensional pain assessment to improve health outcomes in nursing home residents with dementia in Jordan. Also, the findings of this study will inform conducting an experimental study to determine the efficacy of training sessions about the RCP model to improve the critical thinking and decision-making skills of nurses. For both experimental studies, pre-intervention (i.e., pretest) scores for the DS-DAT tool and WAI tool would be collected and compared with post-intervention (i.e., posttest) scores. Inferential statistical tests would then be used to determine if there were any significant differences between the two groups with respect to pain and agitation levels.

\section{Chapter Summery}

This chapter summarized the main findings of the study. This chapter also discussed the implications of this study for nursing education, clinical nursing practice and policy that could contribute to the nursing science of pain assessment and treatment in PWD. This chapter discussed the conceptual and methodological limitations of this study. Finally, this chapter

presented some recommendations for future research about the assessment and treatment of pain in PWD. 


\section{References}

Achterberg, W. P., Pieper, M. J., van Dalen-Kok, A. H., de Waal, M. W., Husebo, B. S., Lautenbacher, S., ... \& Corbett, A. (2013). Pain management in patients with dementia. Clinical Interventions in Aging, 8, 1471.

Adunsky, A., Levy, R., Mizrahi, E., \& Arad, M. (2002). Exposure to opioid analgesia in cognitively impaired and delirious elderly hip fracture patients. Archives of Gerontology and Geriatrics, 35(3), 245-251.

Al-Adawi, S., Braidy, N., Essa, M., Al-Azri, F., Hussain, S., Al-Sibani, N., ... \& Al-Mashani, A. (2014). Cognitive profiles in patients with multi-infarct dementia: an omani study. Dementia and Geriatric Cognitive Disorders Extra, 4(2), 271.

Al-btoush, A. (2012). Relation between the Psychological Impact of Presbycusis and Hearing Handicap Inventory for the Elderly Using Screening Version in Jorda. Middle East Journal of Age and Ageing, 9(5), 23-25.

Al-Harthy, M., Ohrbach, R., Michelotti, A., \& List, T. (2015). The effect of culture on pain sensitivity. Journal of Oral Rehabilitation, doi: 10.1111/joor.12346

Ali, N., Luther, S. L., Volicer, L., Algase, D., Beattie, E., Brown, L. M., ... \& Joseph, I. (2015). Risk assessment of wandering behavior in mild dementia. International Journal of Geriatric Psychiatry, doi: 10.1002/gps.4336.

Aliyu, U., Mathew, O. K., Paul, R. Y., Shinaba, S. T., Oyewole, W. R., \& Olusanya, M. O. (2014). Promoting professional nursing practice through critical thinking and attitudinal change. Journal of Nursing and Health Science, 3(5), 12-15. 
Allen, J. (2001, April 30). Untreated, Chronic Pain Is a Major Problem for Nursing Home Residents. Retrieved April 25, 2015, from http://articles.latimes.com/2001/apr/30/health/he-57458

Almomani, F., Hamasha, A. A. H., Williams, K. B., \& Almomani, M. (2013). Oral health status and physical, mental and cognitive disabilities among nursing home residents in Jordan. Gerodontology.

Almomani, F. M., McDowd, J. M., Bani-Issa, W., \& Almomani, M. (2014). Health-related quality of life and physical, mental, and cognitive disabilities among nursing home residents in Jordan. Quality of Life Research, 23(1), 155-165.

Bani-Issa, W., Almomani, F., \& Eldeirawi, K. (2014). Urinary incontinence among adult women with diabetes in Jordan: epidemiology, correlates and perceived impact on emotional and social well-being. Journal of Clinical Nursing, 23(17-18), 2451-2460.

Bankole, A., Anderson, M., Smith-Jackson, T., Knight, A., Oh, K., Brantley, J., ... \& Lach, J. (2012). Validation of noninvasive body sensor network technology in the detection of agitation in dementia. American Journal of Alzheimer's Disease and Other Dementias, $27(5), 346-354$

Barber, J. B., \& Gibson, S. J. (2009). Treatment of chronic non-malignant pain in the elderly. Drug Safety, 32(6), 457-474.

Black, B. S., Finucane, T., Baker, A., Loreck, D., Blass, D., Fogarty, L., ... \& Rabins, P. V. (2006). Health problems and correlates of pain in nursing home residents with advanced dementia. Alzheimer Disease \& Associated Disorders, 20(4), 283-290. 
Borson, S., Scanlan, J. M., Lessig, M., \& DeMers, S. (2010). Comorbidity in aging and dementia: scales differ, and the difference matters. The American Journal of Geriatric Psychiatry, 18(11), 999-1006.

Boult, C., \& Wieland, G. D. (2010). Comprehensive primary care for older patients with multiple chronic conditions:“Nobody rushes you through”. JaMa, 304(17), 1936-1943.

Bowirrat, A., Cui, J., Waraska, K., Friedland, R. P., Oscar-Berman, M., Farrer, L. A., ... \& Baldwin, C. T. (2005). Lack of association between angiotensin-converting enzyme and dementia of the Alzheimer's type in an elderly Arab population in Wadi Ara, Israel. Neuropsychiatric Disease and Treatment, 1(1), 73.

Boyd, C. M., McNabney, M. K., Brandt, N., Correa-de-Araujuo, R., Daniel, M., Epplin, J., ... \& Shega, J. W. (2012). Guiding principles for the care of older adults with multimorbidity: an approach for clinicians: American Geriatrics Society Expert Panel on the Care of Older Adults with Multimorbidity. J Am Geriatr Soc, 60(10), E1-E25

Brecher, D. B., \& West, T. L. (2014). Underrecognition and Undertreatment of Pain and Behavioral Symptoms in End-Stage Dementia. American Journal of Hospice and Palliative Medicine, 1049909114559069.

Browning, C., Sims, J., Kendig, H., \& Teshuva, K. (2009). Predictors of physical activity behavior in older community-dwelling adults. Journal of Allied Health, 38(1), 8-17.

Cader, R., Campbell, S., \& Watson, D. (2005). Cognitive Continuum Theory in nursing decision $\square$ making. Journal of Advanced Nursing, 49(4), 397-405.

Callister, L. C. (2003). Cultural influences on pain perceptions and behaviors. Home Health Care Management \& Practice, 15(3), 207-211. 
Cervo, F. A., Raggi, R. P., Bright-Long, L. E., Wright, W. K., Rows, G., Torres, A. E., ... \& Komaroff, E. (2007). Use of the certified nursing assistant pain assessment tool (CPAT) in nursing home residents with dementia. American Journal of Alzheimer's Disease and Other Dementias, 22, 112-119.

Chang, S. O., Oh, Y., Park, E. Y., Kim, G. M., \& Kil, S. Y. (2011). Concept analysis of nurses' identification of pain in demented patients in a nursing home: development of a hybrid model. Pain Management Nursing, 12(2), 61-69.

Clark, L., Jones, K., \& Pennington, K. (2004). Pain assessment practices with nursing home residents. Western Journal of Nursing Research, 26(7), 733-750.

Cohen, D. (2013). End-of-Life Issues for Caregivers of Individuals with Alzheimer's Disease and Related Dementias. In Caregiving for Alzheimer's Disease and Related Disorders (pp. 121-135). Springer New York.

Cohen-Mansfield, J., \& Billig, N. (1986). Agitated behaviors in the elderly: a conceptual review. Journal of the American Geriatrics Society.

Congedo, M., Causarano, R. I., Alberti, F., Bonito, V., Borghi, L., Colombi, L., ... \& Gasparini, M. (2010). Ethical issues in end of life treatments for patients with dementia. European Journal of Neurology, 17(6), 774-779.

Damush, T. M., Kroenke, K., Bair, M. J., Wu, J., Tu, W., Krebs, E. E., \& Poleshuck, E. (2016). Pain self-management training increases self-efficacy, self-management behaviours and pain and depression outcomes. European Journal of Pain.

Dawson, R., \& Lavori, P. W. (2008). Sequential causal inference: Application to randomized trials of adaptive treatment strategies. Statistics in Medicine, 27(10), 1626. 
Davidhizar, R., \& Giger, J. N. (2004). A review of the literature on care of clients in pain who are culturally diverse. International Nursing Review, 51(1), 47-55.

Dening, K. H. (2014). Observational pain assessment in advanced dementia. Nursing and Residential Care, 16(7), 378-382.

Dewing, J. (2010). Responding to agitation in people with dementia. Nursing Older People, $22(6), 18-25$.

Dhami, M. K., \& Thomson, M. E. (2012). On the relevance of Cognitive Continuum Theory and quasirationality for understanding management judgment and decision making. European Management Journal, 30(4), 316-326.

Eid, T., Manias, E., Bucknall, T., \& Almazrooa, A. (2014). Nurses' knowledge and attitudes regarding pain in Saudi Arabia. Pain Management Nursing, 15(4), e25-e36.

Ersek, M., Herr, K., Neradilek, M. B., Buck, H. G., \& Black, B. (2010). Comparing the psychometric properties of the checklist of nonverbal pain behaviors (CNPI) and the pain assessment in advanced dementia (PAINDAD) instruments. Pain Medicine, 11(3), 395-

404.

Feldt, K. S. (2000). The checklist of nonverbal pain indicators (CNPI). Pain Management Nursing, 1(1), 13-21.

Fine, P. G. (2009). Chronic pain management in older adults: special considerations. Journal of Pain and Symptom Management, 38(2), S4-S14.

Flaskerud, J. H. (2015). Pain and culture: the catastrophizing construct and measurement. Issues in Mental Health Nursing, 36(2), 152.

Gibson, S. J., \& Lussier, D. (2012). Prevalence and relevance of pain in older persons. Pain Medicine, 13(s2), S23-S26. 
Gilmore-Bykovskyi, A. L., \& Bowers, B. J. (2013). Understanding nurses' decisions to treat pain in nursing home residents with dementia. Research in Gerontological Nursing, 6(2), 127138.

Gloth 3rd, F. M. (2000). Geriatric pain. Factors that limit pain relief and increase complications. Geriatrics, 55(10), 46-8.

Gustavsson, E., \& Sandman, L. (2015). Health-care needs and shared decision-making in priority-setting. Medicine, Health Care and Philosophy, 18(1), 13-22.

Haasum, Y., Fastbom, J., Fratiglioni, L., Kåreholt, I., \& Johnell, K. (2011). Pain treatment in elderly persons with and without dementia. Drugs \& Aging, 28(4), 283-293.

Hadjistavropoulos, T., Herr, K., Prkachin, K. M., Craig, K. D., Gibson, S. J., Lukas, A., \& Smith, J. H. (2014). Pain assessment in elderly adults with dementia. The Lancet Neurology, 13(12), 1216-1227.

Hadjistavropoulos, T., Herr, K., Turk, D. C., Fine, P. G., Dworkin, R. H., Helme, R., ... \& Williams, J. (2007). An interdisciplinary expert consensus statement on assessment of pain in older persons. The Clinical Journal of Pain, 23, S1-S43.

Herr, K. A., \& Garand, L. (2001). Assessment and measurement of pain in older adults. Clinics in Geriatric Medicine, 17(3), 457-478.

Herr, K., Coyne, P. J., McCaffery, M., Manworren, R., \& Merkel, S. (2011). Pain assessment in the patient unable to self-report: position statement with clinical practice recommendations. Pain Management Nursing, 12(4), 230-250.

Horgas, A. L. (2003). Assessing pain in older adults with dementia. Pain, 126(3), 210-220.

Horgas, A. \& Miller, L. (2008). Pain assessment in people with dementia. AJN The American Journal of Nursing, 108(7), 62-70. 
Huffman, J. C., \& Kunik, M. E. (2000). Assessment and understanding of pain in patients with dementia. The Gerontologist, 40(5), 574-581.

Hulley, S. B., Cummings, S. R., Browner, W. S., Grady, D. G., \& Newman, T. B. (2013). Designing Clinical Research. Lippincott Williams \& Wilkins.

Hung, W. W., Liu, S., \& Boockvar, K. S. (2010). A prospective study of symptoms, function, and medication use during acute illness in nursing home residents: design, rationale and cohort description. BMC Geriatrics, 10(1), 1-8.

Hurley, A. C., Volicer, B. J., Hanrahan, P. A., Houde, S., \& Volicer, L. (1992). Assessment of discomfort in advanced Alzheimer patients. Research in Nursing \& Health, 15(5), 369377.

Husebo, B. S., Ballard, C., \& Aarsland, D. (2011). Pain treatment of agitation in patients with dementia: a systematic review. International Journal of Geriatric Psychiatry, 26(10), 1012-1018.

Inelmen, E. M., Mosele, M., Sergi, G., Toffanello, E. D., Coin, A., \& Manzato, E. (2012). Chronic pain in the elderly with advanced dementia. Are we doing our best for their suffering?. Aging Clinical and Experimental Research, 24(3), 207-212.

James, I. A. (2011). Understanding behaviour in dementia that challenges: a guide to assessment and treatment. Jessica Kingsley Publishers.

Johnson, R., \& Taylor, C. (2011). Can playing pre-recorded music at mealtimes reduce the symptoms of agitation for people with dementia?. International Journal of Therapy and Rehabilitation, 18(12), 700-708.

Kaasalainen, S. (2007). Pain assessment in older adults with dementia: using behavioral observation methods in clinical practice. Journal of Gerontological Nursing, 33(6), 6-10. 
Kaasalainen, S., Brazil, K., Ploeg, J., \& Martin, L. S. (2007). Nurses' perceptions around providing palliative care for long-term care residents with dementia. Journal of Palliative Care, 23(3), 173-180.

Kaasalainen, S., Coker, E., Dolovich, L., Papaioannou, A., Hadjistavropoulos, T., Emili, A., \& Ploeg, J. (2007). Pain management decision making among long-term care physicians and nurses. Western Journal of Nursing Research, 29(5), 561-580.

Knebel, M., Haberstroh, J., Kümmel, A., Pantel, J., \& Schröder, J. (2015). CODEMamb-an observational communication behavior assessment tool for use in ambulatory dementia care. Aging \& Mental Health, 1-11.

Koller, D. (2011). Death knell for the lecture: Technology as a passport to personalized education. New York Times, 5.

Kong, E. H. (2005). Agitation in dementia: concept clarification. Journal of Advanced Nursing, 52(5), 526-536.

Kovach, C. R. (2013). Assessing pain and unmet need in patients with advanced dementia: the role of the Serial Trial Intervention (STI). In Handbook of Pain and Palliative Care (pp. 131-144). Springer New York.

Kovach, C. R., Cashin, J. R., \& Sauer, L. (2006). Deconstruction of a complex tailored intervention to assess and treat discomfort of people with advanced dementia. Journal of Advanced Nursing, 55(6), 678-688.

Kovach, C. R., Griffie, J., Muchka, S., Noonan, P. E., \& Weissman, D. E. (2000). Nurses' perceptions of pain assessment and treatment in the cognitively impaired elderly: it's not a guessing game. Clinical Nurse Specialist, 14(5), 215-220. 
Kovach, C. R., Hekel, B., \& Rababa, M. (2015). Feasibility Testing of a Protocol to Stop Ineffective Drug and Nondrug Treatments. Western Journal of Nursing Research, 37(11), 1404-1422.

Kovach, C. R., Noonan, P. E., Griffie, J., Muchka, S., \& Weissman, D. E. (2001). Use of the assessment of discomfort in dementia protocol. Applied Nursing Research, 14(4), 193200.

Kovach, C.R., Noonan, P.E., Schlidt, A.M. \& Wells, T. (2005). A model of consequences of need-driven dementia-compromised behavior (C-NDB). Journal of Nursing Scholarship, 37, 134-140.

Kovach, C.R., Logan, B.R., Joosse, L.L., \& Noonan, P.E. (2012b). Failure to Identify Behavioral Symptoms of People with Dementia and the Need for Follow-Up Physical Assessment. Research in Gerontological Nursing, 5(2), 89-93. PMC3161167

Kovach, C.R., Logan, B., Noonan, P.E., Schlidt, A.M., Smerz, J., Simpson, M., \& Wells, T. (2006). Effects of the serial trial intervention on discomfort and behavior in demented nursing home residents. American Journal of Alzheimer's Disease \& Other Dementias, 21(3), 147-155.

Kovach, C.R., Logan, B.R., Simpson, M., \& Reynolds, S. (2010). Factors associated with time to identify physical problems of nursing home residents with dementia. American Journal of Alzheimer's Disease \& Other Dementias, 25(4), 317-323. (PMID: 20237337; PMCID: PMC2884077).

Kovach, C. R., Simpson, M. R., Joosse, L., Logan, B. R., Noonan, P. E., Reynolds, S. A., ... \& Raff, H. (2012). Comparison of the effectiveness of two protocols for treating nursing 
home residents with advanced dementia. Research in Gerontological Nursing. 5(4), 251-263. PMID: 22998656; PMCID: PMC3475742.

Krumm, N., Larkin, P., Connolly, M., Rode, P., \& Elsner, F. (2014). Improving dementia care in nursing homes: experiences with a palliative care symptom-assessment tool (MIDOS). International Journal of Palliative Nursing, 20(4), 187-192.

Kunz, M., Lautenbacher, S., LeBlanc, N., \& Rainville, P. (2012). Are both the sensory and the affective dimensions of pain encoded in the face? Pain, 153(2), 350-358.

Lally, J., \& Tullo, E. (2012). Engaging older people in decisions about their healthcare: the case for shared decision making. Reviews in Clinical Gerontology, 22(02), 99-107.

Lasch, K. E. (2000). Culture, pain, and culturally sensitive pain care. Pain Management Nursing, $1(3), 16-22$.

Lauri, S., \& Salantera, S. (2002). Developing an instrument to measure and describe clinical decision making in different nursing fields. Journal of Professional Nursing, 18(2), 93100.

Lichtner, V., Dowding, D., Esterhuizen, P., Closs, S. J., Long, A. F., Corbett, A., \& Briggs, M. (2014). Pain assessment for people with dementia: a systematic review of systematic reviews of pain assessment tools. BMC geriatrics, 14(1), 138.

Lin, C. F., LeBoulluec, A. K., Zeng, L., Chen, V. C., \& Gatchel, R. J. (2014). A decision-making framework for adaptive pain management. Health Care Management Science, 17(3), 270-283.

Lints-Martindale, A. C., Hadjistavropoulos, T., Lix, L. M., \& Thorpe, L. (2012). A comparative investigation of observational pain assessment tools for older adults with dementia. The Clinical Journal of Pain, 28(3), 226-237. 
Liu, J. Y., Briggs, M., \& Closs, S. J. (2010). The psychometric qualities of four observational pain tools (OPTs) for the assessment of pain in elderly people with osteoarthritic pain. Journal of Pain and Symptom Management, 40(4), 582-598.

LoGiudice, D., \& Watson, R. (2014). Dementia in older people: an update. Internal Medicine Journal, 44(11), 1066-1073.

Lovering, S. (2006). Cultural attitudes and beliefs about pain. Journal of Transcultural Nursing, 17(4), 389-395.

Lukas, A., Schuler, M., Fischer, T. W., Gibson, S. J., Savvas, S. M., Nikolaus, T., \& Denkinger, M. (2012). Pain and Dementia. Zeitschrift für Gerontologie und Geriatrie, 45(1), 45-49.

Machanic, M. (2001). Faculty development in higher education: "Best practices" review and planning recommendations for technology-rich learning environments. DEOSNEWS.

Malloy, D. C., \& Hadjistavropoulos, T. (2004). The problem of pain management among persons with dementia, personhood, and the ontology of relationships. Nursing Philosophy, 5(2), $147-159$.

Manfredi, P. L., Breuer, B., Wallenstein, S., Stegmann, M., Bottomley, G., \& Libow, L. (2003). Opioid treatment for agitation in patients with advanced dementia. International Journal of Geriatric Psychiatry, 18(8), 700-705.

McCaffery, M., Ferrell, B. R., \& Pasero, C. (2000). Nurses' personal opinions about patients' pain and their effect on recorded assessments and titration of opioid doses. Pain Management Nursing, 1(3), 79-87.

McDonald, D. D. (2014). Trialing to pain control: a grounded theory. Research in Nursing \& Health, 37(2), 107-116. 
McLachlan, A. J., Bath, S., Naganathan, V., Hilmer, S. N., Le Couteur, D. G., Gibson, S. J., \& Blyth, F. M. (2011). Clinical pharmacology of analgesic medicines in older people: impact of frailty and cognitive impairment. British Journal of Clinical Pharmacology, 71(3), 351-364.

McIlfatrick, S. (2015). Palliative care in dementia: literature review of nurses' knowledge andattitudes towards pain assessment. International Journal of Palliative Nursing, 21(8), 400-407.

Mohammad, H., Kassim, N., Yasir, A. (2013). Older Adult Social Needs Nursing Home. Medical Journal of Babylon, 10(3), p. 625-631. Retrieved November 6, 2014 from: http://www.uobabylon.edu.iq/uobColeges/fileshare/articles/repository1_publication16063 27 2922.pdf.

Monroe, T. B., Parish, A., \& Mion, L. C. (2015). Decision factors nurses use to assess pain in nursing home residents with dementia. Archives of Ppsychiatric Nursing, 29(5), 316-320.

Monroe, T., Carter, M., Feldt, K., Tolley, B., \& Cowan, R. L. (2012). Assessing advanced cancer pain in older adults with dementia at the end-of-life. Journal of advanced nursing, 68(9), 2070-2078.

Offredy, M., Kendall, S., \& Goodman, C. (2008). The use of cognitive continuum theory and patient scenarios to explore nurse prescribers' pharmacological knowledge and decisionmaking. International Journal of Nursing Studies, 45(6), 855-868.

Palmer, R. F., \& Royall, D. R. (2010). Missing data? Plan on it!. Journal of the American Geriatrics Society, 58(s2), S343-S348. 
Papp, K. K., Huang, G. C., Clabo, L. M. L., Delva, D., Fischer, M., Konopasek, L., ... \& Gusic, M. (2014). Milestones of critical thinking: a developmental model for medicine and nursing. Academic Medicine, 89(5), 715-720.

Parke, B. (1998). Gerontological nurses' ways of knowing. Realizing the presence of pain in cognitively impaired older adults. Journal of Gerontological Nursing, 24(6), 21-28.

Parker, T. Y. (2013). Management of chronic non-malignant pain in nursing homes residents. Journal of Student Nursing Research, 5(1), 4.

Patel, K. V., Guralnik, J. M., Dansie, E. J., \& Turk, D. C. (2013). Prevalence and impact of pain among older adults in the United States: Findings from the 2011 National Health and Aging Trends Study. PAIN, 154(12), 2649-2657.

Polit, D. F., \& Beck, C. T. (2008). Nursing research: Generating and assessing evidence for nursing practice. Lippincott Williams \& Wilkins.

Pretz, J. E., \& Folse, V. N. (2011). Nursing experience and preference for intuition in decision making. Journal of Clinical Nursing, 20(19】20), 2878-2889.

Price, B. (2015). Applying critical thinking to nursing. Nursing Standard, 29(51), 49-60.

Reynolds, K. S., Hanson, L. C., DeVellis, R. F., Henderson, M., \& Steinhauser, K. E. (2008). Disparities in pain management between cognitively intact and cognitively impaired nursing home residents. Journal of Pain and Symptom Management, 35(4), 388-396.

Rozzini, R., Frisoni, G. B., Ferrucci, L., Barbisoni, P., Sabatini, T., Ranieri, P., ... \& Trabucchi, M. (2002). Geriatric index of comorbidity: validation and comparison with other measures of comorbidity. Age and Ageing, 31(4), 277-285.

Salem, M. (2009). Health for all: Inequity in health policies in the Arab countries. Paper presented at the IUSSP international seminar. London, UK. 
Shega, J., Emanuel, L., Vargish, L., Levine, S. K., Bursch, H., Herr, K., ... \& Weiner, D. K. (2007). Pain in persons with dementia: complex, common, and challenging. The Journal of Pain, 8(5), 373-378.

Shega, J. W., Hougham, G. W., Stocking, C. B., CoxDHayley, D., \& Sachs, G. A. (2006).

Management of noncancer pain in community $\square$ dwelling persons with dementia. Journal of the American Geriatrics Society, 54(12), 1892-1897.

Shields, C. G., Finley, M. A., Elias, C. M., Coker, C. J., Griggs, J. J., Fiscella, K., \& Epstein, R. M. (2013). Pain Assessment: the roles of physician certainty and curiosity. Health Communication, 28(7), 740-746.

Smebye, K. L., Kirkevold, M., \& Engedal, K. (2012). How do persons with dementia participate in decision making related to health and daily care? A multi-case study. BMC Health Services Research, 12(1), 241.

Snow, A. L., Chandler, J. F., Kunik, M. E., Davila, J. A., Balasubramanyam, V., Steele, A. B., \& Morgan, R. O. (2009). Self-reported pain in persons with dementia predicts subsequent decreased psychosocial functioning. The American Journal of Geriatric Psychiatry, 17(10), 873-880.

Sternberg, S., Bentur, N., \& Shuldiner, J. (2014). Quality of care of older people living with advanced dementia in the community in Israel. Journal of the American Geriatrics Society, 62(2), 269-275.

Tait, R. C., Chibnall, J. T., \& Kalauokalani, D. (2009). Provider judgments of patients in pain: seeking symptom certainty. Pain Medicine, 10(1), 11-34. 
Tanner, C. A. (2006). Thinking like a nurse: A research-based model of clinical judgment in nursing. Journal of Nursing Education, 45(6), 204-211.

Thuathail, A. N., \& Welford, C. (2011). Pain assessment tools for older people with cognitive impairment. Nursing Standard, 26(6), 39-46.

Tracy, B., \& Morrison, R. S. (2013). Pain management in older adults. Clinical Therapeutics, $35(11), 1659-1668$.

Van Der Steen, J. T., Pasman, H. R. W., Ribbe, M. W., Van Der Wal, G., \& OnwuteakaPhilipsen, B. D. (2009). Discomfort in dementia patients dying from pneumonia and its relief by antibiotics. Scandinavian Journal of Infectious Diseases, 41(2), 143-151.

Victor, T. W., Jensen, M. P., Gammaitoni, A. R., Gould, E. M., White, R. E., \& Galer, B. S. (2008). The dimensions of pain quality: factor analysis of the Pain Quality Assessment Scale. The Clinical Journal of Pain, 24(6), 550-555.

White, S. M., Wójcicki, T. R., \& McAuley, E. (2012). Social cognitive influences on physical activity behavior in middle-aged and older adults. Journals of Gerontology: Series B: Psychological Sciences And Social Sciences, 67B(1), 18-26.

Wein, S. (2011). Impact of culture on the expression of pain and suffering. Journal of Pediatric Hematology/Oncology, 33, S105-S107.

Weissman, D. E., Gordon, D., \& Bidar-Sielaff, S. (2004). Cultural aspects of pain management. Journal of Palliative Medicine, 7(5), 715-717.

Woods, B., \& Moniz-Cook, E. (2012). Dementia: pain relief - a first-line response to agitation in dementia?. Nature Reviews Neurology, 8(1), 7-8.

Wu, N., Miller, S. C., Lapane, K., Roy, J., \& Mor, V. (2005). Impact of cognitive function on assessments of nursing home residents' pain. Medical Care, 43(9), 934-939. 
Zekry, D., Valle, B. H. L., Lardi, C., Graf, C., Michel, J. P., Gold, G., ... \& Herrmann, F. R. (2010). Geriatrics index of comorbidity was the most accurate predictor of death in geriatric hospital among six comorbidity scores. Journal of Clinical Epidemiology, 63(9), 1036-1044.

Zieber, C.,G.,Hagen, B., Armstrong-Esther, C., Aho, M. (2005). Pain and agitation in long-term care residents with dementia: use of the Pittsburgh Agitation Scale. International Journal of Palliative Nursing, 11 (2), 71-8.

Zimmerman, S., Williams, C. S., Reed, P. S., Boustani, M., Preisser, J. S., Heck, E., \& Sloane, P. D. (2005). Attitudes, stress, and satisfaction of staff who care for residents with dementia. The Gerontologist, 45(1), 96-105.

Zwakhalen, S. M., Hof, C. E., \& Hamers, J. P. (2012). Systematic pain assessment using an observational scale in nursing home residents with dementia: exploring feasibility and applied interventions. Journal of Clinical Nursing, 21(21-22), 3009-3017. 


\section{APPENDICES \\ Appendix A: \\ Discomfort Scale for Dementia of Alzheimer's Type (DS-DAT)}

Behavioral Indicators for Discomfort Scale Items

Rating (Circle One)

Noisy breathing: negative sounding noise on inspiration or expiration; breathing looks

strenuous, labored, or wearing; respirations sound loud, harsh, or gasping; difficulty

breathing or trying hard at attempting to achieve a good gas exchange: episodic bursts

NONE

of rapid breaths or hyperventilation.

Negative vocalization: noise or speech with a negative or disapproving quality; hushed low sounds such as constant muttering with a guttural tone; monotone, subdued, or varying pitched noise with a definite unpleasant sound; faster rate than a conversation or drawn out as in a moan or groan; repeating the same words with a mournful tone; expressing hurt or pain.

Content facial expression: pleasant calm looking face; tranquil, at ease, or serene; relaxed facial expression with a slack unclenched jaw; overall look is one of peace.

Sad facial expression: troubled looking face; looking hurt, worried, lost, or lonesome; distressed appearance; sunken, "hang dog' look with lackluster eyes; tears; crying.

Frightened facial expression: scared, concerned looking face: looking bothered. fearful or troubled; alarmed appearance with open eyes \& pleading face.

NONE

Frown: face looks strained; stern or scowling looks; displeased expression with a wrinkled brow and creases in the forehead; corners of mouth turned down.

NONE

Relaxed body language: easy openhanded position; look of being in a restful position and may be cuddled up or stretched out; muscles look of normal firmness and joints are without stress; look of idle, lazy, or "laid back:" appearance of "just killing the day"; casual.

Tense body language: extremities show tension; wringing hands, clenched fist, or knees pulled up tightly; look of being in a strained and inflexible position.

NONE 
Appendix B:

SCORING SHEET

Wisconsin Agitation Inventory

WAI

SUBJECT \#

RATER

口 PRE

DATE

\begin{tabular}{|l|l|lllll|}
\hline \multicolumn{1}{|c|}{$\begin{array}{c}\text { Time } \\
\text { Scheduled }\end{array}$} & $\begin{array}{c}\text { Time } \\
\text { Collected }\end{array}$ & \multicolumn{5}{c|}{ Agitation Scale Score } \\
\hline Breakfast & & 0 & 25 & 50 & 75 & 100 \\
\hline Breakfast & 0 & 25 & 50 & 75 & 100 \\
\hline Midmornin & & 0 & 25 & 50 & 75 & 100 \\
g & & 0 & 25 & 50 & 75 & 100 \\
\hline Midmornin & & 0 & 25 & 50 & 75 & 100 \\
g & & 0 & 25 & 50 & 75 & 100 \\
\hline Predinner & & 0 & 25 & 50 & 75 & 100 \\
\hline Predinner & & 0 & 25 & 50 & 75 & 100 \\
\hline Postdinner & & & & & & \\
\hline Postdinner & & & & & & \\
\hline
\end{tabular}

Agitation Intensity Parameters

\begin{tabular}{|c|c|c|c|l|}
\hline $\begin{array}{c}\text { Scor } \\
\mathbf{e}\end{array}$ & $\begin{array}{c}\text { \# of } \\
\text { Behavior } \\
\mathbf{s}\end{array}$ & $\begin{array}{c}\text { Duration of } \\
\text { Behaviors }\end{array}$ & $\begin{array}{c}\text { And } / \\
\text { Or }\end{array}$ & \multicolumn{1}{|c|}{ Intensity } \\
\hline 0 & 0 & --- & --- & The person was entirely calm. \\
\hline 25 & 1 & $\leq 15$ seconds & AND & Minimal motor, verbal or vocal behavior. \\
\hline 50 & 1 & $16-59$ seconds & OR & $>$ Minimal motor, verbal or vocal behavior. \\
\hline 50 & 2 & $\leq 15$ seconds & AND & Minimal motor, verbal or vocal behavior. \\
\hline 75 & 1 & $60-119$ seconds & AND & Minimal motor, verbal or vocal behavior. \\
\hline 75 & 2 & $16-59$ seconds & AND & Minimal motor, verbal or vocal behavior. \\
\hline 100 & 1 & $\geq 120$ seconds & OR & High motor, verbal or vocal behavior. \\
\hline 100 & $\geq 2$ & $\geq 60$ seconds & OR & High motor, verbal or vocal behavior. \\
\hline
\end{tabular}




\section{Measuring Agitation}

Agitated behavior is verbal, vocal, or motor behavior that is not explained by the events (e.g. need of the situation) per se. In other words, in a non-demented person in a similar situation, the behavior would not be expected. Agitation is an excited or aroused state.

Look at the random order table to determine the sequence of observing residents. Since we don't observe residents in the toilet, or behind a closed privacy curtain, you may adapt this sequence to ensure feasible data collection. Observe the subject for 3 minutes. Please try to capture all behaviors and don't underestimate behaviors that you can explain away or potentially "fix."

Place a slash mark on the line to indicate how agitated the subject has been over the previous 3 minutes. Here are some definitions of what marks at various points along the line indicate:

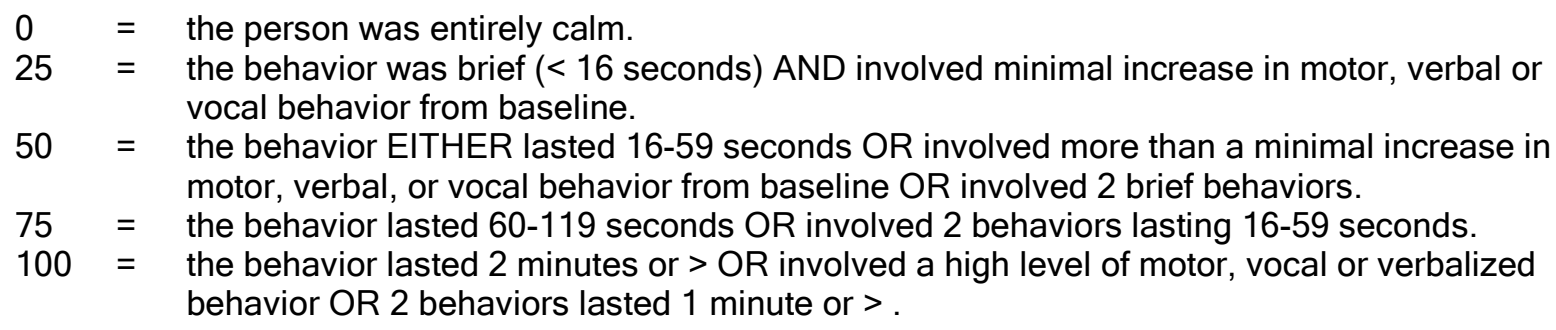

The maximum score for each line is a 100. For example, if a subject displayed two behaviors (i.e. move repetitively for 10 seconds and scratch for 50 seconds) the score would be a 75 since the 2 behaviors lasted 60 seconds. If the person bit another person for 10 seconds the score would be 100 since this is a high level of motor activity that is not usual.

EXAMPLES OF AGITATED BEHAVIORS

1. Complaining- whining, complaining about self, physical complaints, personal gripes or complaining about external things or other people.

2. Negativism-bad attitude, doesn't like anything, nothing is right

3. General Restlessness- fidgeting, always moving around in seat, getting up and sitting down, inability to sit still

4. Pacing and Aimless Wandering- constantly walking back and forth, does not indicate normal purposeful walk, include wandering when done in a wheelchair

5. Cursing or Verbal Aggression- swearing, unkind speech or criticism, verbal anger, verbal combativeness

6. Constant Unwarranted Request for Attention or Help- verbal or nonverbal unreasonable nagging, pleading, demanding

7. Repetitive Sentences or Questions- repeating the same sentence or question one right after the other

8. Inappropriate Dressing or Disrobing- putting on too many clothes, putting on clothing in a strange manner, taking off clothing in public or when it is inappropriate

\footnotetext{
- Used with permission of J. Cohen-Mansfield.
} 
9. Spitting- spitting onto floor or other people, include spitting while feeding. Do not include salivation over which the person has no control or spitting into tissue, the toilet, or onto the ground outside

10. Hitting (including self)- physical abuse, striking, pinching, banging self/furniture

11. Kicking- strike forcefully with feet at people or objects

12. Grabbing Onto People or Things Inappropriately- snatching, seizing roughly, taking firmly, or yanking

13. Pushing- forcefully thrusting, shoving, moving, putting pressure against

14. Throwing Things- hurl, violently tossing in the air

15. Making Strange Noises- including crying, weeping, moaning, weird laughter, grinding teeth

16. Screaming- loud, shrill, shouting, piercing howl

17. Biting- chomp, gnash, gnaw (people or self)

18. Eating or Drinking Inappropriate Substance- putting into mouth and trying to swallow items that are inappropriate

19. Scratching- clawing, scraping with fingernails (people or self)

20. Trying to Get to a Different Place- trying to get out of the building, off the property, sneaking out of the room, leaving inappropriately, trying to get into locked areas, trespassing within unit, other people's room or closet

21. Intentional Falling- purposefully falling onto floor, include from wheelchair, floor or bed

22. Hurting Self or Other- burning self or other, cutting self or other, touching self or other with harmful objects

23. Handling Things Inappropriately- picking up things that don't belong to them, rummaging through drawers, moving furniture, playing with food, fecal smearing

24. Hiding Things- putting objects under or behind something

25. Tearing Things or Destroying Property- shredding, ripping, breaking, stomping on something

26. Performing Repetitious Mannerisms- patting, tapping, rocking self, fiddling with something, rubbing self or object, sucking fingers, taking shoes on and off, picking at self, clothing or objects, picking at imaginary things out of air or off floor, manipulation of nearby objects in a repetitious manner

27. Making Verbal Sexual Advances- sexual propositions, sexual innuendo, or "dirty" talk

28. Making Physical Sexual Advances or Exposing Genitals- touching a person in an inappropriate sexual way, rubbing genital area, inappropriate masturbation when not alone in own room or bathroom, unwanted fondling or kissing

29. Hoarding Things- putting many or inappropriate objects in purse or pockets, keeping too many of an ite 


\title{
Appendix C:
}

\section{The Mini-Mental State Examination \\ Score

\section{Orientation}

1. What is the:

\author{
Year? \\ Season? \\ Date? \\ Day? \\ Month?
}

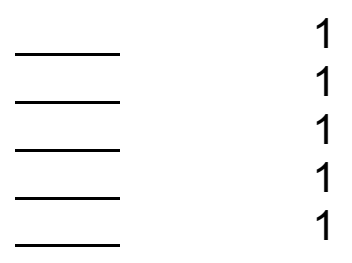

2. Where are we?
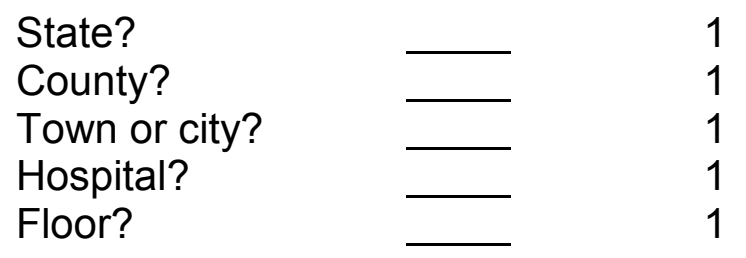

\section{Registration}

3. Name three objects, taking one second to say each. Then ask the patient all three after you have said them. Give one point for each correct answer. Repeat the answers until the patient learns all three.

\section{Attention and calculation}

4. Serial sevens. Give one point for each correct answer. Stop after five answers. Alternate: Spell WORLD backwards.

\section{Recall}

5. Ask for names of three objects learned in Question 3. Give one point for each correct answer.

\section{Language}

6. Point to a pencil and a watch. Have the patient name them as you point. 
7. Have the patient repeat "No ifs, ands, or buts."

8. Have the patient follow a three-stage command: "Take the paper in your right hand. Fold the paper in half. Put the paper on the floor." paper on the floor."

Have the patient read and obey the following: "CLOSE YOUR. EYES." (Write in large letters.)

10. Have the patient write a sentence of his or her own choice. (The sentence should contain a subject and a verb and should make sense. Ignore spelling errors when scoring.)

11. Enlarge the design printed below to 1 inch per side and have the patient copy it. (Give one point if all sides and angles are preserved and if the intersecting sides form a quadrangle.)

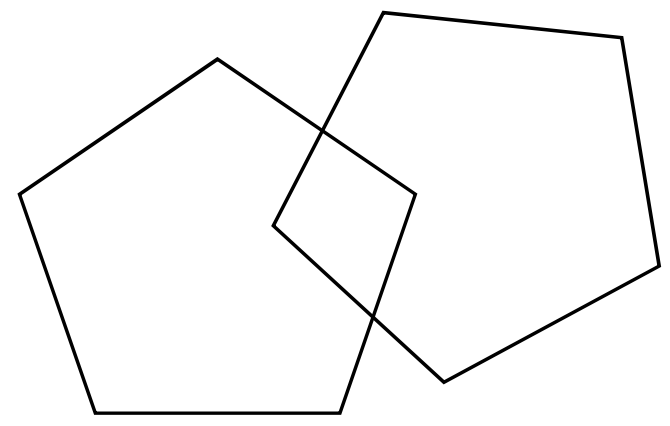

Total Points

Reprinted from Journal of Psychiatric Research, Vol. 12, Folstein, M.F., Folstein, S.E., \& McHugh, P.R., "Mini-Mental State: A practical method for grading the cognitive state of patients for the clinician," 189-198, Copyright (1975), with permission from Pergamon Press Ltd, Headington Hill Halt, Oxford OX3 OBW, U 
Instructions for Administration of Mini-Mental Status Examination

Orientation: Ask for the date. Then ask specifically for parts omitted, e.g., "Can you also tell me what season it is?" One point for each correct answer. Ask in turn "Can you tell me the name of this hospital?" (nursing home, town, county, etc.). One point for each correct answer.

Registration: Ask the patient if you may test his memory. Then say the names of 3 unrelated objects, clearly and slowly, about one second for each. After you have said a 3 , ask him to repeat them. This first repetition determines his score (0-3) but keep saying them until he can repeat all 3 , up to 6 trials. If he does not eventually learn all 3 recall (see below) cannot be meaningfully tested.

Attention and Calculation: Ask the patient to begin with 100 and count backwards by 7. Stop after 5 subtractions $(93,86,79,72,65)$. Score the total number of correct answers. If the patient cannot or will not perform this task, ask him to spell the word "world" backwards. The score is the number of letters in correct order. E.g. dlrow $=5$, dlorw $=3$.

Recall: Ask the patient if he can recall the 3 words you previously asked him to remember. Score 0-3.

Language:

Naming: Show the patient a pencil and ask him what it is. Repeat for wrist watch. Score $0-2$, one for each correct answer, 0 if none are named.

Repetition: Ask the patient to repeat the sentence after you. Allow only one trial. Score 0 or 1 .

3-Stage command: Give the patient a piece of plain blank paper and repeat the entire command. Score 1 point for each part correctly executed.

Reading: On a blank piece of paper print the sentence, "Close your eyes," in letters large enough for the patient to see clearly. Ask him to read it and do what it says. Score 1 point only if he actually closes his eyes.

Writing: Give the patient a blank piece of paper and ask him to write a sentence for you. Do not dictate a sentence; it is to be written spontaneously. It must contain a subject and verb and be sensible. Correct grammar and punctuation are not necessary.

Copying: On a clean piece of paper, draw intersecting pentagons, each side about 1 inch, and ask him to copy it exactly as it is. All 10 angles must be present and 2 must intersect to score 1 point. Tremor and rotation are ignored.

Level of Consciousness: Estimate the patient's level of sensorium along a continuum, from alert on the left to coma on the righ 


\section{Appendix D: \\ SCORING SHEET \\ Cumulative IIIness Rating Scale for Geriatrics \\ $\operatorname{CIRS}(\mathrm{G})$}

\section{SUBJECT \#}

RATER

DATE

Instructions. Please refer to the CIRS (G) Manual. Write brief descriptions of the medical problem(s) that justified the endorsed score on the line following each item. (Use the reverse side for more writing space). Rating Strategy

0- No problem

1- Current mild problem or past significant problem

2- Moderate disability or morbidity/requires "first line" therapy

3- Sever/constant significant disability/"uncontrollable" chronic problems

4- Extremely severe/immediate treatment required/end organ failure/severe impairment function

\begin{tabular}{|c|c|}
\hline \multirow{2}{*}{ Heart } & Score \\
\hline & \\
\hline \multicolumn{2}{|l|}{ Vascular } \\
\hline \multicolumn{2}{|l|}{ Hematopoietic } \\
\hline \multicolumn{2}{|l|}{ Respiratory } \\
\hline \multicolumn{2}{|l|}{ Eyes, ears, nose, throat, and larynx } \\
\hline \multicolumn{2}{|l|}{ Upper gastrointestinal tract } \\
\hline \multicolumn{2}{|l|}{ Lower gastrointestinal tract } \\
\hline \multicolumn{2}{|l|}{ Liver } \\
\hline \multicolumn{2}{|l|}{ Renal } \\
\hline \multicolumn{2}{|l|}{ Genitourinary } \\
\hline \multicolumn{2}{|l|}{ Musculoskeletal/Integument } \\
\hline \multicolumn{2}{|l|}{ Neurological } \\
\hline \multicolumn{2}{|l|}{ Endocrine/Metabolic } \\
\hline \multicolumn{2}{|l|}{ Psychiatric Illness } \\
\hline \multicolumn{2}{|l|}{ Total Number Categories Endorsed } \\
\hline \multicolumn{2}{|l|}{ Total Score } \\
\hline \multicolumn{2}{|l|}{ Severity Index: (total score/total number of categories endorsed) } \\
\hline \multicolumn{2}{|l|}{ Number of categories at level-3 severity } \\
\hline Number of categories at level-4 severity & \\
\hline
\end{tabular}




\section{Change Tracking Form}

Subject \#:

Date:

Time:

Description of behaviors changes/changes in condition:

For both questions below, please circle the response that characterizes your answer, where $1=$ very uncertain, $2=$ uncertain, $3=$ don't know, $4=$ certain, and $5=$ very certain

1. Prior to your assessment, what is your level of certainty that the person has physical pain and/or discomfort as a result of changes?
Very Certain
Certain
Don't know
Uncertain
Very Uncertain

5

4

3

2

1

2. After your assessment, what is your level of certainty that the person has physical pain and discomfort as a result of changes?

$\begin{array}{ccccc}\text { Very Certain } & \text { Certain } & \text { Don't know } & \text { Uncertain } & \text { Very Uncertain } \\ 5 & 4 & 3 & 2 & 1\end{array}$

What are drug and non-drug treatments given to the person? 


\title{
Appendix F:
}

UNIVERSITY of WISCONSIN

UUMILWAUKEE

Department of University Safety \& Assurances

\section{New Study - Notice of IRB Expedited Approval}

\author{
Meliess Spadaneda \\ IRB Manager \\ Instiunsional Revice Roent \\ Enselmenn 270 \\ P. O. Box 413 \\ Malarakee, WI 53201-0413 \\ (414) 229-3173 phone \\ (414) 229-6729 kx

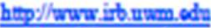 \\ spodanud arexrmedn
}

Date: January 12, 2016

To: Christine Kovach $\mathrm{PhD}$

Dept: Nursing

Ce: Mohammad Rababa

IRB\#: 16.182

Title: The Association of Nurses' Assessment and Certainty to Pain Management and Outcomes for Nursing Home Residents in Jordan

After review of your research protocol by the University of Wisconsin - Milwaukee Institutional Review Board your protocol has been approved as minimal risk Expedited under Category 7 as governed by 45 CFR 46.110 .

This protocol has been approved on January 12, 2016 for one year. IRB approval will expire on January 11,2017 . If you plan to continue any research related activities (e.g., enrollment of subjects, study interventions, data analysis, etc.) past the date of $\mathbb{R B}$ expiration, a continuation for IRB approval must be filed by the submission deadline. If the study is closed or completed before the IRB expiration date, please notify the IRB by completing and submitting the Continuing Review form found in IRBManager.

Any proposed changes to the protocol must be reviewed by the IRB before implementation, unless the change is specifically necessary to eliminate apparent immediate hazards to the subjects. It is the principal investigator's responsibility to adhere to the policies and guidelines set forth by the UWM $I R B$, maintain proper documentation of study records and promptly report to the $R B$ any adverse events which require reporting. The principal investigator is also responsible for ensuring that all study staff receive appropriate training in the ethical guidelines of conducting human subjects research.

As Phincipal Investigator, it is your responsibility to adhere to UWM and UW System Policies, and any applicable state and federal laws governing activities which are independent of $I R B$ review/approval (e.g., FERPA, Radiation Safety, UWM Data Security, UW System policy on Prizes, Awards and Gifts, state gambling laws, etc.). When conducting research at institutions outside of UWM, be sure to obtain permission and/or approval as required by their policies.

Contact the IRB office if you have any further questions. Thank you for your cooperation and best wishes for a successful project.

Respectfully,

meviac Cinadasuda

Melissa C. Spadanuda

IRB Manager 


\author{
Appendix G: \\ Resident's Consent Form \\ UNIVERSITY OF WISCONSIN - MILWAUKEE \\ CONSENT TO PARTICIPATE IN RESEARCH \\ RESIDENT CONSENT
}

\begin{abstract}
THIS CONSENT FORM HAS BEEN APPROVED BY THE IRB FOR A ONE YEAR PERIOD]
\end{abstract}

\title{
1. General Information
}

Study title: The Association of Nurses' Assessment and Certainty to Pain Management and Outcomes for Nursing Home Residents in Jordan

Person in Charge of Study (Principal Investigator):

- Mohammad Rababa PhDC, RN. He is a PhD student at UWM/CON

\section{Study Description}

You are being asked to participate in a research study. Your participation is completely voluntary. You do not have to participate if you do not want to.

\section{Study description:}

Pain is sometimes not noticed by nurses and not treated well enough. Nurses may not be sure if a person has a pain and this can delay treatment. The purpose of this study is to look at relationships between how sure the nurse is about a person's pain, the assessment the nurse does to determine if pain exists and how well patients are feeling.

This study is being done at three nursing homes in Amman. We are trying to get 76 nursing home residents with some memory problems to participate. If you agree to participate it means we will review your medical record and collect information about your pain and agitation level before, during, and after the time you attend the meeting with Mr. Rababa. The meeting will be one time to answer some memory questions that should take 10 minutes or less. These questions will cover areas such as: orientation, recall, attention and calculation, and language . Response \#2: After a stressful event, you may be observed for the level of pain and agitation by M. Rababa or the RA two times for 30 minutes each time. Both observations will be done midday in the nursing home where you reside.

\section{Study Procedures}

What will I be asked to do if I participate in the study? 
If you agree to participate will not be asked to do anything except answering some common memory questions. These questions will cover areas such as: orientation, recall, attention and calculation, and language. After a stressful event, you will be observed for the level of pain and agitation by M. Rababa or the RA two times for 30 minutes each time. Both observations will be done midday in the nursing home where you reside. No audio/video/photographic recordings will be done.

\section{Risks and Minimizing Risks}

What risks will I face by participating in this study?

Risk to participating in this study is minimal. To the best of our knowledge, and from other studies done in this area, participants did not experience any problems

\section{Benefits}

Will I receive any benefit from my participation in this study?

There are no benefits to you other than to further research.

\section{Study Costs and Compensation}

Will I be charged anything for participating in this study?

You will not be responsible for any of the costs from taking part in this research study.

Are subjects paid or given anything for being in the study?

The subject will receive token of appreciation upon the completion of the study.

\section{Confidentiality}

\section{What happens to the information collected?}

All information collected about you during the study will be kept confidential to the extent permitted by law. We may present what we find to others, or publish our results in scientific journals or at scientific conferences. Only the PI (Mohammad Rababa) will have access to the information. However, the Institutional Review Board at UW-Milwaukee or appropriate federal agencies like the Office for Human Research Protections may review this study's records.

- You will be assigned an identification number (ID). Confidentiality will be maintained by using ID numbers instead of your name.

- M. Rababa will keep records in a locked, secured location accessible only to him. Computer data will be coded by ID number and will not contain identifying information.

- Data will be stored on a password-protected computer. Only M. Rababa will have 
access to code numbers that link information to your name.

- M. Rababa will store hard copies of any forms used in a locked file cabinet in a locked office for 5 years for possible future use.

\section{Alternatives}

Are there alternatives to participating in the study?

There are no known alternatives available to you other than not taking part in this study.

\section{Voluntary Participation and Withdrawal}

What happens if I decide not to be in this study?

Your participation in this study is entirely voluntary. You may choose not to take part in this study. If you decide to take part, you can change your mind later and withdraw from the study. You are free to not answer any questions or withdraw at any time or after 2 times of displeasure with observations. Your decision will not change any present or future relationships with the University of Wisconsin Milwaukee. If the subject withdraws or is withdrawn early, we will use the information collected to that point.

\section{Questions}

\section{Who do I contact for questions about this study?}

For more information about the study or the study procedures or treatments, or to withdraw from the study, contact:

Mohammad Rababa, PhDC, RN

College of Nursing

500 W. Hampton Ave, apt 307

Glendale, WI 53217

mrababa@uwm.edu

Who do I contact for questions about my rights or complaints towards my treatment as a research subject?

The Institutional Review Board may ask your name, but all complaints are kept in confidence.

Institutional Review Board

Human Research Protection Program

Department of University Safety and Assurances

University of Wisconsin - Milwaukee

P.O. Box 413

Milwaukee, WI 53201 


\section{Signatures}

\section{Research Subject's Consent to Participate in Research:}

To voluntarily agree to take part in this study, you must sign on the line below. If you choose to take part in this study, you may withdraw at any time. You are not giving up any of your legal rights by signing this form. Your signature below indicates that you have read or had read to you this entire consent form, including the risks and benefits, and have had all of your questions answered, and that you are 18 years of age or older.

Printed Name of Subject/ Legally Authorized Representative

Signature of Subject/Legally Authorized Representative

Date

\section{Research Subject's Consent to Audio/Video/Photo Recording:}

\section{Principal Investigator (or Designee)}

I have given this research subject information on the study that is accurate and sufficient for the subject to fully understand the nature, risks and benefits of the study.

Printed Name of Person Obtaining Consent

Signature of Person Obtaining Consent
Study Role

Date 


\section{Appendix H \\ Proxy Consent Form \\ UNIVERSITY OF WISCONSIN - MILWAUKEE \\ CONSENT TO PARTICIPATE IN RESEARCH \\ FAMILY MEMBER/PROXY CONSENT}

THIS CONSENT FORM HAS BEEN APPROVED BY THE IRB FOR A ONE YEAR PERIOD

\section{General Information}

Study title: The Association of Nurses' Assessment and Certainty to Pain Management and Outcomes for Nursing Home Residents in Jordan

Person in Charge of Study (Principal Investigator):

- Mohammad Rababa $\mathrm{PhD}(\mathrm{C}, \mathrm{RN}$. He is a $\mathrm{PhD}$ student at UWM/CON

\section{Study Description}

We are interested in having your family member, , participate in a research study being conducted at the nursing home where he resides. Their participation is completely voluntary and will not affect any care or ability to participate in any activities offered at the nursing Home.

\section{Study description:}

Pain is sometimes not noticed by nurses and not treated well enough. Nurses may not be sure if a person has a pain and this can delay treatment. The purpose of this study is to look at relationships between how sure the nurse is about a person's pain, the assessment the nurse does to determine if pain exists and how well patients are feeling.

This study is being done at three nursing homes in Amman. We are trying to get 76 nursing home residents with some memory problems to participate. If you agree to have your family member participate it means we will review their medical record and collect information about their pain and agitation level before, during, and after the time they attend the meeting with Mr. Rababa. The meeting will be one time to answer some memory questions that should take 10 minutes or less. These questions will cover areas such as: orientation, recall, attention and calculation, and language. After a stressful event, your family member may be observed for the level of pain and agitation by M. Rababa or the RA two times for 30 minutes each time. Both observations will be done midday in the nursing home where they reside.

\section{Study Procedures}

What will your family member be asked to do if I participate in the study? 
If you agree to have your family member participate, they will not be asked to do anything except answering some common memory questions. These questions will cover areas such as: orientation, recall, attention and calculation, and language. After a stressful event, your family member will be observed for the level of pain and agitation by M. Rababa or the RA two times for 30 minutes each time. Both observations will be done midday in the nursing home where they reside. No audio/video/photographic recordings will be done.

\section{Risks and Minimizing Risks}

What risks will your family member face by participating in this study?

Risk to participating in this study is minimal. To the best of our knowledge, and from other studies done in this area, participants did not experience any problems

\section{Benefits}

Will my family member receive any benefit from participation in this study?

- By participating in this study we will gain more information about possible barriers to assessment and treatment of pain in your family member. If we find any of these possible barriers, the information will be shared with their doctor or nurse.

\section{Study Costs and Compensation}

\section{Will I be charged anything for this study?}

- You or your family member, or any of their insurance or benefit provider's will not be responsible for any of the costs from taking part in this research study.

\section{Are subjects paid or given anything for being in the study?}

- You or your family member will not be given any money or gifts to take part in this research study.

\section{Confidentiality}

\section{What happens to the information collected?}

All information collected about your family member during the course of this study will be kept confidential to the extent permitted by law. We may decide to present what we find to others, or publish our results in scientific journals or at scientific conferences. Only the PI and his assistant will have access to the information. However, the Institutional Review Board at UW-Milwaukee or appropriate national agencies like the Office for Human Research Protections may review this study's records. 
- The information we collect will be recorded with a code number---not with the resident's name attached. We will be able to link this code number to their name through a list kept by M. Rababa and his RA.

- M. Rababa will keep records in a locked, secured location accessible only to him. Computer data will be coded by ID number and will not contain identifying information.

- Data will be stored on a password-protected computer. Only M. Rababa will have access to code numbers that link information to your name.

- M. Rababa will store hard copies of the completed data sheets in a locked file cabinet in a locked office for 5 years for possible future use.

\title{
8. Alternatives
}

Are there alternatives to participating in the study?

- There are no known alternatives available to your family member other than not taking part in this study.

\section{Voluntary Participation and Withdrawal}

What happens if you decide not to have your family member be in this study? Your family member's participation in this study is entirely voluntary. You may choose not to have your family member take part in this study. If you decide to take part now, you can change your mind later and withdraw your family member from the study. Your family member are free to withdraw at any time or after 2 times of displeasure with observations. Your decision will not change any present or future relationships with the nursing home. If you withdraw from the study, we may use the information collected to that point.

\section{Questions}

Who do I contact for questions about this study?

For more information about the study or the study procedures or treatments, or to withdraw from the study, contact:

\author{
Mohammad Rababa, $\mathrm{PhD} \odot$, $\mathrm{RN}$ \\ College of Nursing \\ 500 W. Hampton Ave, apt 307 \\ Glendale, WI 53217 \\ mrababa@uwm.edu
}

Who do I contact for questions about my rights or complaints towards my treatment as a
research subject?
The Institutional Review Board may ask your name, but all complaints are kept in confidence.

Institutional Review Board 
Human Research Protection Program

Department of University Safety and Assurances

University of Wisconsin - Milwaukee

P.O. Box 413

Milwaukee, WI 53201

(414) 229-3173

\section{Signatures}

\section{Research Subject's Consent to Participate in Research:}

To voluntarily agree to have your family member take part in this study, you must sign on the line below. If you choose to have your family member take part in this study, you may withdraw at any time. You are not giving up any of your legal rights by signing this form. Your signature below indicates that you have read or had read to you this entire consent form, including the risks and benefits, and have had all of your questions answered, and that you are 18 years of age or older.

Printed Name of Subject/ Legally Authorized Representative

Signature of Subject/Legally Authorized Representative

Date

\section{Principal Investigator (or Designee)}

I have given this research subject information on the study that is accurate and sufficient for the subject to fully understand the nature, risks and benefits of the study.

Printed Name of Person Obtaining Consent

Signature of Person Obtaining Consent
Study Role

Date 


\section{Appendix I \\ Nurse Consent Form \\ UNIVERSITY OF WISCONSIN - MILWAUKEE \\ CONSENT TO PARTICIPATE IN RESEARCH \\ NURSE CONSENT}

THIS CONSENT FORM HAS BEEN APPROVED BY THE IRB FOR A ONE YEAR PERIOD]

\section{General Information}

Study title: The Association of Nurses' Assessment and Certainty to Pain Management and Outcomes for Nursing Home Residents in Jordan

Person in Charge of Study (Principal Investigator):

- Mohammad Rababa MSN, RN. I am a PhD student at UWM/CON

\section{Study Description}

You are being asked to participate in a research study. Your participation is completely voluntary. You do not have to participate if you do not want to.

\section{Study description:}

Pain in people with dementia (PWD) is clinically challenging to assess because they often have both a cognitive impairment and communication problems that interfere with their ability to communicate effectively and clearly with their family members, nurses or physicians. These problems of pain assessment in PWD may make nurses uncertain about pain in PWD. The purpose of this project is to study relationships between a PWDs change in behavior, whether the nurse thinks the person is having pain, the types of assessments nurses do, and pain and agitation in PWD. The study is being done at three local nursing homes. We expect that approximately 812 nurses will participate from the three nursing homes. Your participation in the study will occur over 2-3 months.

\section{Study Procedures}

\section{What will I be asked to do if I participate in the study?}

If you agree to participate, M. Rababa will ask you to start filling out Change Tracking forms once you notice change in residents' behaviors or condition. M. Rababa will train you to start the tracking form for any resident who has a change in behavior or condition. Examples of common behavior changes seen in residents will be taught and include those associated with pain (e.g. facial grimacing, restless body movement) as well as changes in behavior that are less commonly associated with pain (e.g. change in appetite, decreased interest in activities). You will mark on 
the form whether you think the resident is having any pain prior to your assessment. Then you will do your regular assessment and document that assessment on the Change Tracking Form. After that, you will again mark on the form whether you think the resident has any physical pain or discomfort. M. Rababa and the research assistant will measure the resident's pain and agitation levels 3 and 7 days after the change in residents' condition. Both measurements will be done midday because it is more convenient for residents and you

\section{Risks and Minimizing Risks}

What risks will I face by participating in this study?

There are no foreseeable risks for participating in this research study.

\section{Benefits}

Will I receive any benefit from my participation in this study?

There are no benefits to you other than to further research.

\section{Study Costs and Compensation}

Will I be charged anything for participating in this study?

You will not be responsible for any of the costs from taking part in this research study.

Are subjects paid or given anything for being in the study?

The subject will receive token of appreciation upon the completion of the study.

\section{Confidentiality}

\section{What happens to the information collected?}

All information collected about you during the course of this study will be kept confidential to the extent permitted by law. We may decide to present what we find to others, or publish our results in scientific journals or at scientific conferences. Only Mr. Rababa will have access to the information. However, the Institutional Review Board at UW-Milwaukee or appropriate federal agencies like the Office for Human Research Protections may review this study's records.

- You will be assigned an identification number (ID). Confidentiality will be maintained by using ID numbers instead of your name.

- M. Rababa will keep records in a locked, secured location accessible only to him.

- Computer data will be coded by ID number and will not contain identifying information. Data will be stored on a password-protected computer.

- Only M. Rababa will have access to code numbers that link information to your name. 
- M. Rababa will store hard copies of the completed data sheets in a locked file cabinet in a locked office for 5 years for possible future use.

\section{Alternatives}

Are there alternatives to participating in the study?

There are no known alternatives available to you other than not taking part in this study.

\section{Voluntary Participation and Withdrawal}

What happens if I decide not to be in this study?

Your participation in this study is entirely voluntary. You may choose not to take part in this study. If you decide to take part, you can change your mind later and withdraw from the study. You are free to not answer any questions or withdraw at any time. Your decision will not change any present or future relationships with the University of Wisconsin Milwaukee. If the subject withdraws or is withdrawn early, we will use the information collected to that point.

\section{Questions}

\section{Who do I contact for questions about this study?}

For more information about the study or the study procedures or treatments, or to withdraw from the study, contact:

Mohammad Rababa, PhDC, RN

College of Nursing

500 W. Hampton Ave, apt 307

Glendale, WI 53217

\section{Who do I contact for questions about my rights or complaints towards my treatment as a research subject?}

The Institutional Review Board may ask your name, but all complaints are kept in confidence.

Institutional Review Board

Human Research Protection Program

Department of University Safety and Assurances

University of Wisconsin - Milwaukee

P.O. Box 413

Milwaukee, WI 53201

(414) 229-3173

\section{Signatures}




\section{Research Subject's Consent to Participate in Research:}

To voluntarily agree to take part in this study, you must sign on the line below. If you choose to take part in this study, you may withdraw at any time. You are not giving up any of your legal rights by signing this form. Your signature below indicates that you have read or had read to you this entire consent form, including the risks and benefits, and have had all of your questions answered, and that you are 18 years of age or older.

Printed Name of Subject/ Legally Authorized Representative

$\overline{\text { Signature of Subject/Legally Authorized Representative }}$

Date

Research Subject's Consent to Audio/Video/Photo Recording:

Principal Investigator (or Designee)

I have given this research subject information on the study that is accurate and sufficient for the subject to fully understand the nature, risks and benefits of the study.

Printed Name of Person Obtaining Consent

Signature of Person Obtaining Consent
Study Role

Date 


\section{CURRICULUM VITAE}

\section{LICENSURE AND CERTIFICATION}

\section{MOHAMMAD RABABA}

2011-present Basic Life Support Instructor, American Heart Association

2008-present Jordanian Registered Nurse

\section{EDUCATION}

University of Wisconsin-Milwaukee

Ph.D. in Nursing, December 2016

University of Wisconsin-Milwaukee

Graduate Non-degree Certificate, April 2014

University of Virginia, Charlottesville

M.S. in Nursing, December 2012

CNS

University of Virginia, Charlottesville

Adult-Gerontology CNS Certificate, December 2012

Jordan University of Science and Technology, Jordan

B.S. in Nursing, June 2008
Major GPA: 3.970/4.0
Minor: Gerontology
Major GPA: 4.0/4.0
Minor: Applied Gerontology
Major GPA: 3.496/4.0
Minor: Adult-Gerontology

Major GPA: 3.496/4.0

Minor: Neurotrauma

Major GPA: 3.94/4.0

\section{PROFESSIONAL AND TEACHING EXPERIENCEE}

University of Wisconsin-Milwaukee/ School of Nursing Teaching Assistant $\quad$ August, 2014May, 2016

- Teach and supervised for approximately 100 undergraduate students in different nursing courses including: Aging and Long Term Care and Health Promotion, Cultural Diversity in Healthcare.

- Grade exams, quizzes, and papers, and to provide class support to the faculty.

University of Wisconsin-Milwaukee/ School of Nursing Clinical Project Manager Jan16, 2014-Aug 16, 2014

- I provided clinical data management support to study project (T3 project). It is a study of factors that are associated with overuse of proton pump inhibitor medications by nursing home residents. Our contention was that the overuse of proton pump inhibitors is a part of a prescribing cascade in which polypharmacy and overuse of vitamins, minerals and supplements begets the need for proton pump inhibitors to treat dyspepsia (i.e. upper stomach upset).

- I participated in the review of Clinical research documents (e.g. Protocols, Case 
Report Forms, Reports and Statistical analysis).

- I developed tracking sheets and codebook and participated in collecting and coding data, entering them to SPSS, and analyzing them.

ADURO Screening Company Health Screener and Coach Feb-March, 2014

- I was responsible for performing health-screening test including blood test (e.g. cholesterol, blood sugar, HDL, LDL), blood pressure and heart rate, and weigh, height and waist circumference.

- I was responsible for recorded test results and discussed any concerns about the result.

Jordan University of Science and Technology, Jordan Teaching Assistant 2009-2010

- Taught and supervised for approximately 100 undergraduate students in different nursing clinical courses including: Fundamentals of Nursing Practice, Clinical Physical Assessment in nursing school labs, and Adult Health Nursing I, Adult Health Nursing II in medical surgical units.

- Prepared students using a variety of skills. Assessed the student's ability to manage clinical assignments. Created an environment conducive to learning and determined appropriate patient care assignments for students. Role modeled professional interactions on the care unit. Demonstrated nursing actions. Gave timely and appropriate feedback to students

- Worked as Teaching Assistant in two classes: "Scientific Research in Nursing and Nursing Profession Standards and Code of Ethics" and as a coordinator for the clinical course of "Adult Health Nursing."

King Abdullah University Hospital (KAUH), Jordan $\quad$ Registered Nurse 2008-2009

- Conducted physical assessment, respiratory and heart monitoring of ICU patients.

- Managed medication doses, anesthesia, and ventilatory support.

- Performed several nursing procedures and collaborated with other health care providers and patient families in decision regarding treatment.

- Contributed to ethical decision making of medical care protocols.

\section{GRADUATE CLINICAL ROTATION EXPERIENCES}

Neurosurgery/Epilepsy unit NIMU and NNICU, UVa Hospital, Charlottesville, VA Sep., 2011

- Cared for 15 patients and assisted with cardiac monitoring, patient repositioning, patient morning care, many nursing procedures, conducting physical exam, and history taking.

- Reviewed many treatment guidelines and articles that related to neurotrauma and reviewed many neurotrauma patient records. 
- Developed educational materials that concerned complicated migraine and shared with nursing staffs.

- Performed presentation of two evidence based projects regarding reducing medication errors and fall prevention.

\section{RESEARCH EXPERIENCE}

Feasibility Testing of a Protocol to Stop Ineffective Drug and Non-drug Treatments

January, 2014

The purpose of this preliminary study was to determine the feasibility of the T3 processes for end users and to determine if residents who received assessments of treatment response using the $\mathrm{T} 3$ protocol had differences in assessments, treatments started, and treatments stopped than residents treated with usual care. We also examined differences in communication between prescribers, nurse time, and drug costs between the treatment and control groups.

Proton Pump Inhibitors (PPI) and the Prescribing Cascade

January, 2014

It is a study of factors that are associated with overuse of proton pump inhibitor medications by nursing home residents. Our contention was that the overuse of proton pump inhibitors is a part of a prescribing cascade in which polypharmacy and overuse of vitamins, minerals and supplements begets the need for proton pump inhibitors to treat dyspepsia (i.e. upper stomach upset).

Branching Path Simulations Project

August, 2013

- The purpose of this article is to present a new approach to treatment fidelity using branching simulations. Branching simulations are case scenarios that require the user to generate a series of step-by-step decisions and actions.

- Conducted statistical analysis of an approach used in our experimental research to be sure our treatment was delivered as intended.

- Incorporated branching path strategy simulations into treatment fidelity plans.

- Planned a presentation and manuscript publication.

\section{PROFESSIONAL DEVELOPMENT AND SERVICE}

- GSA 2015 Conference, Orlando, FL, Nov. 18-22, 2015

- Midwest Nursing Research Society (MNRS) Conference in Indianapolis, Indiana in April 17,2015

- Midwest Nursing Research Society (MNRS) Conference in St Louis, MO in March 26, 2014

- Living with Grief: End-of-Life Ethics in Charlottesville, VA in April 24, 2012

- Neuro Core Curriculum, Nursing Education Service in Charlottesville, VA in April 24, 2012

- Progressive Care Core Curriculum- Clinical Application, Life Support Learning Center in 
Charlottesville, VA in April 10, 2012

- Evidence Based Practice Day, Jordan Hall Conference Center in Charlottesville, VA March 30, 2012

- Syllabus Design Workshop, Teaching Resource Center in Charlottesville, VA in April 5, 2012.

\section{PRESENTATION}

- Kovach, C., Hekel, B., \& Rababa, M. (2015). Feasibility Testing of a Protocol to Stop Ineffective Drug and Non-drug Treatments. MNRS 40th Annual Research Conference, Milwaukee, WI, March 17, 2016.

- Rababa, M., Alghassani, A., Kovach, C. (2015). Proton Pump Inhibitors (PPI) and the Prescribing Cascade. GSA 2015 Conference, Orlando, FL, Nov. 18-22, 2015.

- Kovach, C., Hekel, B., \& Rababa, M. (2015). Feasibility Testing of a Protocol to Stop Ineffective Drug and Non-drug Treatments. GSA 2015 Conference, Orlando, FL, Nov. 18-22, 2015.

- Rababa, M., Alghassani, A., Kovach, C. (2015). Proton Pump Inhibitors (PPI) and the Prescribing Cascade. MNRS 39th Annual Research Conference, Indianapolis, IN, April $17^{\text {th }}, 2015$.

- Kovach, C. \& Rababa, M. (2014). Incorporating Branching Path Simulation into Treatment Fidelity Plans, Fall 2014 UWM CON Convocation, Milwaukee, WI, November $4^{\text {th }} 2014$

- Kovach, C. \& Rababa, M. (2014). Incorporating Branching Path Simulation into Treatment Fidelity Plans, MNRS 38th Annual Research Conference, St. Louis, MO, March $17^{\text {th }} 2014$.

\section{PUBLICATION}

- Kovach, C. R., Hekel, B., \& Rababa, M. (2015). Feasibility Testing of a Protocol to Stop Ineffective Drug and Nondrug Treatments. Western Journal of Nursing Research, 37(11), 1404-1422.

- Rababa, M., Al-Ghassani, A. A., Kovach, C. R., \& Dyer, E. M. (2015). Proton Pump Inhibitors and the Prescribing Cascade. Journal of Gerontological Nursing.

- Kovach, C. R., \& Rababa, M. (2013). Using branching simulations in treatment fidelity plans. Research in Gerontological nursing, 7(5), 216-223.

- Kovach, C. R., Hekel, B., \& Rababa, M. (2015, November). TESTING A PROTOCOL TO STOP INEFFECTIVE DRUG AND NON-DRUG TREATMENTS. In GERONTOLOGIST (Vol. 55, pp. 283-283). JOURNALS DEPT, 2001 EVANS RD, CARY, NC 27513 USA: OXFORD UNIV. 
2016 Distinguished Abstract at MNRS 40th Annual Research Conference, Milwaukee, WI, March 17, 2016.

2014-present The Honor Society of Nursing, Sigma Theta Tau International (STTI)

2014-present Honor Society Organization

2014 Helen Bader Age \& Community Award form The Helen Bader school of Social

Welfare at UWM

2010 Nursing College Scholarship for academic Excellence in Nursing Sciences (GPA:

3.94/4.0), Jordan.

2008 Graduated with Highest Honors and received the Student Award for

Excellence in General Health Nursing, Jordan.

2001 El-Hassan Youth Award - Based on academic achievement, volunteering, and leadership skills, Jordan.

2008-Present Jordan Nurse and Midwife Council, Jordan.

The National Center for Diabetes, Endocrinology \& Genetics,

$2008-2010$ Jordan, Volunteer.

- Worked with interdisciplinary diabetes team to develop of the plan of care for the individual patient such as diet and exercise regimens.

- Provided diabetic patients educational materials concerned with healthy eating, activity, appropriate medication protocols, problem solving, healthy coping and strategies for reducing risks and diabetes related complications.

\section{COMPUTER SKILLS}

Proficient in Microsoft Word, PowerPoint, Excel, and Microsoft Access. 